\title{
Spectral Expansions of One-dimensional Periodic Dirac Operators
}

\author{
Boris Mityagin \\ Communicated by Charles Li, received April 9, 2004.
}

\begin{abstract}
We study localization of spectra (Counting Lemma) and $L^{p}$ convergence of spectral decompositions of $1 \mathrm{D}$ periodic Dirac operators. The main technical tool is estimates of the resolvent norms in their dependence on the smoothness of a potential.
\end{abstract}

\section{CONTENTs}

1. Introduction; Statement of the Problem and Main Results.

2. How to Evaluate the Norms of Resolvents of Perturbed Operators

3. Inequalities for Sums of Series Which Majorize the Norms of Resolvent Operators

4. Trapping the Spectrum

5. Estimates of Norms of Resolvent and Related Operators in Complex Plane

6. Spectral Expansions and Their Convergence 162

7. Uniform Convergence of Spectral Decompositions 172

8. $L^{2}$-Unconditional Convergence; Uniform Boundedness of Eigenfunctions 183

9. Comments; Conclusion.

\section{Introduction; Statement of the Problem and Main Results.}

We consider 1D periodic Dirac operators

$$
L=i J \frac{d}{d x}+V ; \quad J=\left(\begin{array}{cc}
1 & 0 \\
0 & -1
\end{array}\right), \quad V=\left(\begin{array}{ll}
0 & p \\
q & 0
\end{array}\right),
$$

1991 Mathematics Subject Classification. Primary 34L40, 42C15; Secondary 47E05.

Key words and phrases. Dirac operator, resolvent, spectral decompositions, uniform and unconditional convergence. 
where $p, q$ are $L^{2}$-functions on $[0,1]$. The operators

$$
L^{0}=i J \frac{d}{d x}
$$

and $L$ act in $\mathcal{H}=H \times H, H=L_{2}[0,1]$, being certainly defined on $H^{1} \times H^{1}$ by

$$
L F=\left(\begin{array}{c}
D f_{1}+p f_{2} \\
q f_{1}-D f_{2}
\end{array}\right)
$$

where $F=\left(\begin{array}{l}f_{1} \\ f_{2}\end{array}\right), f_{i} \in H_{1}, i=1,2$ and $D=i d / d x$.

After Zakharov-Shabat $[\mathbf{2 2 ,} \mathbf{2 3}, \mathbf{2 4}]$ such operators are infinitely related to analysis of the complete integrability of a dynamic system - nonlinear (cubic) Schroedinger equation

$$
i \frac{\partial \psi}{\partial t}=-\frac{\partial^{2} \psi}{\partial^{2} x}+\alpha f \psi^{2} \bar{\psi}, \quad f= \pm 1
$$

with the initial condition

$$
\left.\psi(x, t)\right|_{t=0}=\psi(x)
$$

See details in $[\mathbf{1}]$.

2. In this paper we study the structure (or localization) of the spectrum $\sigma\left(L_{b c}\right)$ under different boundary conditions. Three types of bc are most important.

(a) periodic, or $\mathrm{Per}^{+}: F(0)=F(1)$, i.e. $f_{1}(0)=f_{1}(1), f_{2}(0)=f_{2}(1)$,

(b) antiperiodic, or $\mathrm{Per}^{-}: F(0)=-F(1)$, i.e. $f_{1}(0)=-f_{1}(1), f_{2}(0)=-f_{2}(1)$,

(c) Dirichlet bc, or Dir:

$$
f_{1}(0)=f_{2}(0), \quad f_{1}(1)=f_{2}(1) .
$$

Each of these bc's defines the operator $L_{b c}$, the closure of $L$ from a domain

$$
D_{\text {om }}=\left\{F \in H^{1} \times H^{1}: F \in b c\right\}
$$

in a Hilbert space $\mathcal{H}=L_{2} \times L_{2}$ with a scalar product

$$
\langle F, G\rangle=\frac{1}{2} \int_{0}^{1}\left(f_{1} \overline{g_{1}}+f_{2} \overline{g_{2}}\right) d x .
$$

We could consider other regular bc; see the details in Section 6.1.

In the case of $L^{0}=\left(\begin{array}{cc}D & 0 \\ 0 & D\end{array}\right)$ the following table tells everything about eigenvalues and eigenvectors (eigenfunctions) of $L_{b c}^{0}$.

\begin{tabular}{ccc|cc}
\multirow{2}{*}{$\begin{array}{c}\text { complete system } \\
\text { of eigenvectors in } \mathcal{H}\end{array}$} & $\begin{array}{c}\text { spectrum } \\
\sigma\left(L_{b c}\right)\end{array}$ & \multicolumn{2}{|c}{$\begin{array}{c}\text { multiplicity of } \\
\text { eigenvalues } \pi_{p}\end{array}$} \\
\cline { 4 - 5 } & & & $p$ even & $p$ odd \\
\hline Per $^{+}$ & $e_{p}^{ \pm}, p$ even & $\pi n, n$ even & 2 & 0 \\
Per $^{-}$ & $e_{p}^{ \pm}, p$ odd & $\pi n, n$ odd & 0 & 2 \\
Dir & $u_{k}, k \in \mathbb{Z}$ & $\pi n, n \in \mathbb{Z}$ & 1 & 1 \\
\hline
\end{tabular}

In this table and later on we use the following notations.

$$
\begin{aligned}
e_{p}^{+}=\sqrt{2}\left(\begin{array}{c}
e^{-i \pi_{p} x} \\
0
\end{array}\right), \quad e_{p}^{-} & =\sqrt{2}\left(\begin{array}{c}
0 \\
e^{i \pi_{p} x}
\end{array}\right), \quad u_{p}=\frac{1}{\sqrt{2}}\left(e_{p}^{+}+e_{p}^{-}\right), \\
v_{p} & =\frac{1}{\sqrt{2}}\left(e_{p}^{+}-e_{p}^{-}\right) .
\end{aligned}
$$


3. If $V$ is not zero, it is expected that $\sigma\left(L_{b c}\right)$ is "close" to $\sigma\left(L_{b c}^{0}\right)$. Under the assumption that $V \in H^{1} \mathrm{Y}$. Li and D. McLaughlin gave the following version of Counting Lemma (for convenience, they talk about the case $p=-\bar{q}$.)

For $m=\|V\|_{2}, M=\left\|v \mid H^{1}\right\|$ set $N=2\left[m^{2} \cosh m+3 M \sinh m\right]$. Then $\sigma\left(L_{b c}\right) \subset$ $D^{0} \cup \bigcup_{|k|>N} D_{k}$ where $D^{0}=\{\lambda \in \mathbb{C}:|\lambda|<(2 N+1) \pi / 2\}$, and $D_{k}=\{\lambda \in \mathbb{C}:$ $|\lambda-k \pi|<\pi / 4\}$.

The dimensions of corresponding projectors are the same as for $V=0$.

The restriction $V \in H^{1}$ is critical. Such a statement cannot be given just in terms of $m=\|V\|_{2}$. In general context of operators $L_{b c}$, p. 1-3, let us make the following remark, an apologue of Y. Li and D. McLaughlin observation [14], p. 183, Remark 2. The potential

$$
\widetilde{V}=\left[\begin{array}{cc}
0 & p e^{2 i a x} \\
q e^{-2 i a x} & 0
\end{array}\right], a \text { even, }
$$

is 1-periodic and it has the same $L^{2}$-norm $m(\widetilde{V})=m(V)$ as $V$.

If $L F=\lambda F$ then for $\widetilde{L}=i J \frac{d}{d x}+\widetilde{W}$ we have

$$
\widetilde{L} \widetilde{F}=(\lambda+a) \widetilde{F} \quad \text { with } \quad \widetilde{F}=\left(\begin{array}{c}
f_{1} e^{i a x} \\
f_{2} e^{-i a x}
\end{array}\right) .
$$

Moreover, $a$ is even, and it guarantees that

$$
F \in b c \Rightarrow \widetilde{F} \in b c, \quad b c=\operatorname{Per}^{ \pm} \text {or Dir. }
$$

Therefore, $\sigma_{b c}(\widetilde{L})=\sigma_{b c}(L)+a$. It means, that $L^{2}$-control on the initial disk $D^{0}$ (or another shape) is hardly possible.

4. But we can eliminate counterexample of this type of the multiplication by $e^{i a x}, a$ real, would not be a uniformly bounded family of operators in a corresponding (Hilbert) space of potentials. It leads us with necessity to Hilbert weighted norms

$$
\|V \mid H(\Omega)\|^{2}=\sum\left(\left|p_{k}\right|^{2}+\left|q_{k}\right|^{2}\right) \Omega^{2}(k)<\infty
$$

where

$$
\Omega(0)=1, \quad \Omega(k)=\Omega(-k), \quad \Omega(k) \leqslant \Omega(k+1), \quad \Omega(k) \rightarrow \infty(k \rightarrow \pm \infty) .
$$

We'll prove a Counting Lemma just assuming that $V \in H(\Omega)$.

Moreover, the choice of the initial domain $D^{0}$ in $[\mathbf{2 1}],[\mathbf{1 4}]$ and $[\mathbf{9}]$ as a disc does not distinct roles of real and imaginary parts of spectral points, or how $m=\|v\|_{2}$ and $M=\left\|V \mid H^{1}\right\|$ or $\|V \mid H(\Omega)\|$ restrict spectrum in the directions of real and imaginary axes. Instead of a disc $D^{0}$ we talk about rectangles

$$
\Pi=\{z \in \mathbb{C}:|\operatorname{Re} z| \leqslant X,|\operatorname{Im} z| \leqslant Y\}
$$

and try to give much smaller restrictions on $Y$ than on $X$ in Counting Lemma. One of our main results in this paper is the following Theorem.

THEOREM 1.1. Let $V$ be a periodic potential, $V(x+1)=V(x)$, and $V \in H(\Omega)$, where $\Omega$ is a weight sequence in (1.6).

Then for all bc $=\operatorname{Per}^{ \pm}$, Dir

$$
\sigma\left(L_{b c}\right) \subset \Pi(X ; Y) \cup \bigcup_{|k|>N} D_{k}\left(\delta_{k}\right)
$$


where $Y=K_{1}(m M)^{1 / 2}, X=N+1 / 2, N$ depending on $m, M$ and $B$, and $\delta_{k} \rightarrow 0$,

$$
D_{k}(r)=\{z \in \mathbb{C}:|z-\pi k| \leqslant r\} .
$$

The dimensions of all projectors for $\Pi$ and $D_{k}$ are the same as in the case of zero potential.

The precise statement is given in Section 4, Theorems 4.1 and 4.2.

5 . With complex-valued entries $p$ and $q$ of potential $V$, the operator $L_{b c}$ is not necessarily selfadjoint. Spectral expansions of elements $F \in \mathcal{H}$ are defined now in terms of projectors

$$
P=\frac{1}{2 \pi i} \int_{\partial \Pi}\left(z-L_{b c}\right)^{-1} d z
$$

and

$$
P_{k}=\frac{1}{2 \pi i} \int_{\partial D_{k}}\left(z-L_{b c}\right)^{-1} d z .
$$

TheOrem 1.2. If $(\log k)^{2} / \Omega_{k} \rightarrow 0$ the series

$$
P_{\Pi} F+\sum_{|k|>N} P_{k} F=F
$$

converges uniformly for any $F$ satisfying Dini condition.

If $\sum \Omega_{k}^{-2}<\infty$ and $V \in H(\Omega)$ this series converges unconditionally.

See details in Prop. 7.3 and Thm. 7.4, and Prop. 8.7 and Thm. 8.8.

TheOREM 1.3. Under weak assumption $\Omega_{k} \rightarrow \infty$, the eigenfunctions of $L_{b c}$, normalized in $\mathcal{H}$, are uniformly $L_{\infty}$-bounded. Moreover, for some $K=K(\{\Omega\} ; M)$ if $F \in \operatorname{Im} P_{n}$ or $\operatorname{Im} P_{\Pi}$ its $L_{\infty}$-norm

$$
\|F\|_{\infty} \leqslant K\|F\|_{1} .
$$

See details in Thm. 8.4 and Cor. 8.5.

Proofs of these statements have many technical details which could be of certain interest by themselves. The central role belongs to Proposition 2.4, Ineq. (2.27), which provides estimates of the resolvent norms in complex plane.

1.1. Acknowledgments. Many collegues helped me in this work. In SeptemberOctober 2001, when the analytic core of this work was done, Plamen Djakov (of Sofia Univ., Bulgaria) was a very patient listener of different reasons of the constructions in Sect. 2-4 and a valuable advisor. Charles Li (of Univ. Missouri, Columbia, MO) explained to me some results on Counting Lemma and further developments. Evgeni Semenov (of Voronezh State University, Voronezh, Russia) consulted me on a series of inequalities for Fourier coefficients.

My course on Spectral Analysis of Differential Operators, April-May 2002, in CIMAT, Guanajuato, Mexico, contained a few lecftures with presentation of this work. An advice and shrude questions of listeners, in particular, of Steve Sontz and Maite Fernández Unzueta, led to improvement of this exposition. I am thankful to Victor M. Perez-Abreu and Xavier Gomes-Montes for their hospitality during my visit to CIMAT in Spring 2002 when I wrote a significant part of this paper. 


\section{How to Evaluate the Norms of Resolvents of Perturbed Operators}

Elementary methods of perturbation theory of linear operators are based on resolvent identity

$$
(\lambda-A-T)^{-1}=(\lambda-A)^{-1}\left(1-T(\lambda-A)^{-1}\right)^{-1} .
$$

In our context, if

$$
R_{\lambda}=\left(\lambda-L^{0}-V\right)^{-1}, \quad R_{\lambda}^{0}=\left(\lambda-L^{0}\right)^{-1}
$$

then

$$
R_{\lambda}=R_{\lambda}^{0}\left(1-V R_{\lambda}^{0}\right)^{-1}=\left(1-R_{\lambda}^{0} V\right)^{-1} R_{\lambda}^{0} .
$$

We will use only the first representation.

We omit the subscript $b c$ with understanding that both $L^{0}$ and $L$ are considered in (2.1), (2.2) with the same boundary conditions. Of course these identities are valid only if all the operators are well-defined, or can be properly interpreted.

$$
R_{\lambda}^{0} \text { is well defined }
$$

for $\lambda \notin \sigma\left(L_{b c}^{0}\right)$, see Table, p. 126 .

Moreover, by Parseval's identity

$$
\left\|R_{\lambda}^{0}: L_{2} \rightarrow L_{2}\right\|=\max _{s \in \sigma\left(L_{b c}^{0}\right)} \frac{1}{|\lambda-s|} \leqslant \max _{k \in \mathbb{Z}} \frac{1}{|\lambda-\pi k|} .
$$

Of course, by (2.2) $R_{\lambda}$ would be well defined if

$$
\left\|V R_{\lambda}^{0}\right\|_{2} \leqslant \frac{1}{2}
$$

and therefore

$$
\left\|\left(1-V R_{\lambda}^{0}\right)^{-1}\right\|_{2} \leqslant 2 .
$$

But there are two obstacles here. A potential $V$ is an $L_{2}$-function, so an operator of multiplication

$$
V F=\left(\begin{array}{c}
p f_{2} \\
q f_{1}
\end{array}\right)
$$

does not act in $L_{2}$ although there is a bounded operator from $L_{2} \times L_{2} \rightarrow L_{1} \times L_{1}$ and from $L_{\infty} \times L_{\infty} \rightarrow L_{2} \times L_{2}$ with norms

$$
\|V\|_{2 \rightarrow 1}=\|V\|_{\infty \rightarrow 2}=\|v\|_{2} \leqslant m .
$$

We could compensate (2.8) to get (2.5) by treating $R_{\lambda}^{0}$ as an operator from $L_{2}$ to $L_{\infty}$ so

$$
\left\|V R_{\lambda}^{0}\right\|_{2} \leqslant\left\|R_{\lambda}^{0}\right\|_{2 \rightarrow \infty}\|V\|_{\infty \rightarrow 2} .
$$

Indeed, the following is true.

Proposition 2.1. For any

$$
\begin{gathered}
\lambda \notin \pi \mathbb{Z}, \quad \lambda=\pi \mu, \\
\left\|R_{\lambda}^{0}\right\|_{2 \rightarrow \infty} \leqslant \frac{2}{\pi} A(\mu), A^{2}(\mu)=\sum_{k \in \mathbb{Z}} \frac{1}{|\mu-k|^{2}} .
\end{gathered}
$$


Proof. Whatever $b c$ is, the orthonormal eigenfunctions $\varphi_{k}$ of an operator $L^{0}$ are uniformly bounded, say

$$
\left\|\varphi_{k}\right\|_{\infty} \leqslant 2
$$

(see Table, p. 126). For any

$$
\left(\xi_{k}\right) \in l^{2}, \quad \sum\left|\xi_{k}\right|^{2} \leqslant 1
$$

if $\xi=\sum \xi_{k} \varphi_{k}$

$$
R_{\lambda}^{0} \xi=\sum \frac{\xi_{k}}{\lambda-\pi k} \varphi_{k}
$$

and

$$
\left\|R_{\lambda}^{0} \xi\right\|_{\infty} \leqslant \sum\left|\frac{\xi_{k}}{\lambda-\pi k}\right|\left\|\varphi_{k}\right\|_{\infty} \leqslant \frac{2}{\pi}\left(\sum\left|\xi_{k}\right|^{2}\right)^{1 / 2} A(\mu)
$$

i.e. (2.11) holds.

This is a nice inequality but

$$
A(-\mu)=A(\mu)=A(\mu+1) \quad \text { and } \quad \inf _{0<\mu<1} A^{2}(\mu) \geqslant \frac{1}{(1 / 2)^{2}}+\frac{1}{(1 / 2)^{2}}=8 .
$$

Even moving along real line, we cannot make $\left\|V R_{\lambda}^{0}\right\|$ small just by (2.9) although if $\operatorname{Im} \lambda$ is large enough we can guarantee (2.5).

LEMMA 2.2. Let

$$
\mu=x+i y, \quad|x| \leqslant \frac{1}{2}, \quad \rho=|\mu| .
$$

Then

$$
A^{2}(\mu) \leqslant \frac{3}{\rho^{2}}+\frac{10}{1+\rho} .
$$

Proof of the Lemma and other inequalities for spectral sums of this type is given in Section 3.

This lemma leads us imediately to the following

Proposition 2.3. The spectrum $\sigma\left(L_{b c}\right)$ lies in the horizontal strip

$$
\left\{z \in \mathbb{C}|| \operatorname{Im} z \mid \leqslant 4(1+4 m)^{2}\right\} .
$$

Proof. Inequalities (2.9), (2.11) and (2.17) give us for $\rho \geqslant 1$

$$
\left\|V R_{\lambda}^{0}\right\|_{2} \leqslant \frac{2}{\pi}\left(\frac{\sqrt{3}}{\rho}+\frac{\sqrt{12}}{\rho^{1 / 2}}\right)^{m}<\frac{3.5}{\rho^{1 / 2}} m,
$$

where $m \geqslant\|v\|_{2}$. Therefore, (2.5) holds if

$$
\rho \geqslant(1+4 m)^{2} .
$$

But if $\lambda \notin(2.18)$, i.e. $|\operatorname{Im} \lambda|>4(1+4 m)^{2}$, then certainly $\rho=\frac{1}{\pi}|\lambda|>4(1+4 m)^{2}$. It means that (2.20) and (2.5) hold, and $R_{\lambda}=R_{\lambda}^{0}\left(1-V R_{\lambda}^{0}\right)^{-1}$ is well defined.

We cannot get more (see (2.15) and arguments there) from the function $A(\mu)$ and any attempts to use (2.5). But von Neumann series can still help us if we notice that

$$
(1-K)^{-1}=(1+K)\left(1-K^{2}\right)^{-1}
$$


is well defined if $K$ is a bounded operator, and

$$
\left\|K^{2}\right\|<1, \quad \text { say } \leqslant \frac{1}{2} .
$$

We know by (2.11) and (2.17) that $K=V R_{\lambda}^{0}$ is bounded in $L_{2}$ and

$$
\|K\| \leqslant \frac{2}{\pi}\left(\frac{3}{\rho^{2}}+\frac{12}{1+\rho}\right)^{1 / 2} m .
$$

As before (compare (2.9))

$$
\left\|K^{2}\right\|_{2}=\left\|V R_{\lambda}^{0} V R_{\lambda}^{0}\right\|_{2} \leqslant m\left\|R_{\lambda}^{0} V R_{\lambda}^{0}\right\|_{2 \rightarrow \infty} .
$$

If $(2.24) \leqslant 1 / 2$ then $\lambda$ is a regular point, i.e. $\lambda \notin \sigma\left(L_{b c}\right)$. It makes important for us good estimates of the norm $\left\|R_{\lambda}^{0} V R_{\lambda}^{0}\right\|_{2 \rightarrow \infty}$.

Proposition 2.4. Let a potential $V$ in (1.1) be $H(\Omega)$ function, and

$$
\|V \mid H(\Omega)\| \leqslant M<\infty .
$$

Then, with

$$
\lambda=\pi(n+w), \quad|R e w| \leqslant \frac{1}{2}, \quad|w|=\rho
$$

we have

$$
\left\|R_{\lambda}^{0} V R_{\lambda}^{0}\right\|_{2 \rightarrow \infty} \leqslant C M A(2 ; \mu) A_{1}^{b c}(2 ; \omega ; \mu)
$$

where

$$
A_{1}^{\text {Dir }}(2 ; \omega ; \mu)=\left(\sum_{p \text { even }} \omega_{p}^{2} \frac{\log ^{2}(2+|2 n-p|+\rho)}{(2 n-p)^{2}+\rho^{2}}\right)^{1 / 2}
$$

and

$$
A_{1}^{P e r}(2 ; \omega ; \mu)=\left(\sum_{p \text { even }} \frac{\omega_{p}^{2}}{(2 n-p)^{2}+\rho^{2}}\right)^{1 / 2} .
$$

Proof. First, we'll write all the details in a more complicated case, the Dirichlet case, and then analyze $b c=P e r^{ \pm}$with simpler inequalities.

In Dirichlet case (c), Sect. 1.2, components of vector function $F=\left(\begin{array}{l}f_{1} \\ f_{2}\end{array}\right)$ are coupled in boundary conditions so we cannot reduce analysis to blocks and onecomponent decompositions. (We will do just that in $\mathrm{Per}^{ \pm}$cases and even in Dircase when $q(x)=p(-x)$ ).

We know that

$$
\left\{u_{k}, k \in \mathbb{Z}\right\} \in(1.5)
$$

is an orthonormal basis in $\mathcal{H}=L^{2} \times L^{2}$. With this basis $R_{\lambda}^{0}$ acts as a multiplieroperator, i.e. it is diagonal:

$$
R_{\lambda}^{0} u_{j}=\frac{1}{\lambda-\pi j} u_{j}, \quad \forall j \in \mathbb{Z}, \quad \lambda \notin \pi \mathbb{Z} .
$$

Potentials

$$
V=\left(\begin{array}{ll}
0 & p \\
q & 0
\end{array}\right)
$$


with a loose language could also be constructed as elements of $\mathcal{H}$, or

$$
(p, q) \in \mathcal{H}(\Omega)=H(\Omega) \times H(\Omega),
$$

but for our analysis of $V$ another decomposition of $\mathcal{H}$ is important. We put

$$
\mathcal{H}^{+}=\{p(x), p(-x)\}, p \in H(\Omega), \quad \mathcal{H}^{-}=\{p(x),-p(-x)\}, p \in H(\Omega) .
$$

These two subspaces in $\mathcal{H}$ (or in $\mathcal{H}(\Omega)$ ) are orthogonal, and

$$
\mathcal{H}=\mathcal{H}^{+} \oplus \mathcal{H}^{-}
$$

They have orthonormal bases

$$
U_{m}^{+}=\left(\begin{array}{cc}
0 & e^{-i \pi m x} \\
e^{i \pi m x} & 0
\end{array}\right), \quad m \text { even }
$$

and

$$
U_{m}^{-}=\left(\begin{array}{cc}
0 & e^{-i \pi m x} \\
-e^{i \pi m x} & 0
\end{array}\right), \quad m \text { even, }
$$

and any $V \in \mathcal{H}(\Omega)$ has a decomposition

$$
\begin{gathered}
V=\left(\begin{array}{cc}
0 & p(x) \\
q(x) & 0
\end{array}\right)= \\
=\frac{1}{2}\left[\begin{array}{cc}
0 & p(x)+q(-x) \\
p(-x)+q(x) & 0
\end{array}\right]+\frac{1}{2}\left[\begin{array}{cc}
0 & p(x)-q(-x) \\
-p(-x)+q(x) & 0
\end{array}\right]= \\
=V^{+}+V^{-}=\sum_{m \text { even }} v_{m}^{+} U_{m}^{+}+\sum v_{m}^{-} U_{m}^{-},
\end{gathered}
$$

and

$$
\|V\|_{\Omega}^{2} \asymp \sum_{m}\left(\left|v_{m}^{+}\right|^{2}+\left|v_{m}^{-}\right|^{2}\right) \Omega^{2}(m) .
$$

Now

$$
R_{\lambda}^{0} V R_{\lambda}^{0}=R_{\lambda}^{0} V^{+} R_{\lambda}^{0}+R_{\lambda}^{0} V^{-} R_{\lambda}^{0}
$$

and we'll analyze these two terms separately. Put $g_{m}^{ \pm}=v_{m}^{ \pm} \Omega(m)$ so

$$
v_{m}^{ \pm}=g_{m}^{ \pm} \omega_{m} \text { where } \omega_{m}=\frac{1}{\Omega(m)}
$$

and by $(2.25)$

$$
M^{2} \geqslant\|V\|_{\Omega}^{2}=\left\|g\left|l^{2}\left\|^{2}=\right\| g^{+}\right| l^{2}\right\|^{2}+\left\|g^{-} \mid l^{2}\right\|^{2} .
$$

To continue (2.31) we need to know $U_{m}^{ \pm} u_{j}$.

Case $\mathcal{H}^{+}$as we see from (2.36) and (1.5)

$$
U_{m}^{+} u_{j}=u_{m-j} .
$$

Therefore

$$
u_{j} \stackrel{R_{\lambda}^{0}}{\longrightarrow} \frac{1}{\lambda-\pi j} u_{j} \stackrel{\omega_{m} U_{m}^{+}}{\longrightarrow} \frac{\omega_{m}}{\lambda-\pi j} u_{m-j} \stackrel{R_{\lambda}^{0}}{\longrightarrow} \frac{\omega_{m}}{\lambda-\pi j} \cdot \frac{1}{\lambda-\pi(m-j)} u_{m-j}
$$

For

$$
F=\sum f_{j} u_{j}, \quad\|F\|_{2}^{2}=\sum\left|f_{j}\right|^{2} \leqslant 1,
$$

and

$$
V^{+}=\sum q_{m} \omega_{m} U_{m}^{+}, \quad\left\|V^{+}\right\|_{\Omega}^{2}=\sum\left|q_{m}\right|^{2} \leqslant 1
$$


the image-vector is $(\lambda=\pi \mu)$

$$
R_{\lambda}^{0} V^{+} R_{\lambda}^{0} F=\frac{1}{\pi^{2}} \sum_{m, j} f_{j} q_{m} \frac{\omega_{m}}{(\mu-j)(\mu-m+j)} u_{m-j} .
$$

To avoid problems of convergence, we can consider in (2.45) and (2.46) only finite sums. Any constants (norms) independent on $F, V^{+}$with norms $\leqslant 1$ will give us the norm of $R_{\lambda}^{0} V R_{\lambda}^{0}$ anyway. As in (2.12) we know

$$
\left\|u_{k}\right\|_{\infty} \leqslant 2
$$

therefore,

$$
\pi^{2}\left\|R_{\lambda}^{0} V^{+} R_{\lambda}^{0} F\right\|_{\infty} \leqslant S \equiv \sum_{m, j}\left|f_{j} q_{m} \frac{\omega_{m}}{(\mu-j)(\mu-m+j)}\right|
$$

Notice that

$$
\frac{1}{(\mu-j)(\mu-m+j)}=\left[\frac{1}{\mu-j}+\frac{1}{\mu-m+j}\right] \cdot \frac{1}{2 \mu-m},
$$

and define

$$
c_{m}=\sum_{j} f_{j}\left(\frac{1}{\mu-j}+\frac{1}{\mu-m+j}\right) .
$$

By Cauchy inequality

$$
\left|c_{m}\right|^{2} \leqslant\left(\sum_{j}\left|f_{j}\right|^{2}\right)\left(2 \sum \frac{1}{|\mu-j|^{2}}\right) \leqslant 2 A^{2}(\mu) .
$$

Therefore

$S=\sum_{m} c_{m}\left|q_{m}\right| \frac{\omega_{m}}{|2 \mu-m|} \leqslant\left(\sum c_{m}^{2} g_{m}^{2}\right)^{1 / 2}\left(\sum \frac{\omega_{m}^{2}}{|2 \mu-m|^{2}}\right)^{1 / 2} \leqslant \sqrt{2} A(\mu) A_{1}(n, w)$

where

$$
\mu=n+w, \quad|\operatorname{Re} w| \leqslant \frac{1}{2},
$$

and

$$
A_{1}^{2}(n, w)=\sum_{m \text { even }} \frac{\omega_{m}^{2}}{|2 n-m-2 w|^{2}} .
$$

It proves $(2.27)$ in the Dir-case, $V \in \mathcal{H}^{+}$.

Case $\mathcal{H}^{-}$. Now the images ( $m$ even)

$$
U_{m}^{-} u_{j}=v_{m-j}
$$

are not basis elements, and we need their Fourier decomposition. Notice

$$
v_{k}=\sum\left\langle v_{k}, u_{l}\right\rangle u_{l},
$$

and

$$
\left\langle v_{k}, u_{l}\right\rangle=\frac{1}{2} \cdot \frac{1}{2} \int_{0}^{1}\left[e^{-i(k-l) \pi x}-e^{i(k-l) \pi x}\right] d x= \begin{cases}\frac{i}{\pi} \cdot \frac{1}{k-l} & \text { if } k-l \text { odd } \\ 0 & \text { if } k-l \text { even }\end{cases}
$$


SO

$$
U_{m}^{-} u_{j}=\frac{i}{\pi} \sum_{l+j \text { odd }} \frac{1}{m-j-l} u_{l} .
$$

The sequence (2.44) has a little bit different from now:

$$
\begin{gathered}
u_{j} \stackrel{R_{\lambda}^{0}}{\longrightarrow} \frac{1}{\lambda-\pi j} u_{j} \stackrel{\omega_{m} U_{m}^{-}}{\longrightarrow} \frac{\omega_{m}}{\lambda-\pi j} v_{m-j}= \\
=\frac{\omega_{m}}{\pi(\mu-j)} \frac{i}{\pi} \sum_{l+j \text { odd }} \frac{1}{m-j-l} u_{l} \stackrel{R_{\lambda}^{0}}{\longrightarrow} \frac{i}{\pi^{2}} \sum \frac{1}{m-j-l} \frac{\omega_{m}}{\mu-j} \cdot \frac{1}{\mu-l} u_{l} .
\end{gathered}
$$

Again, as in (2.48) $\left\|u_{l}\right\|_{\infty} \leqslant 2$, and with $F \in(2.45)$ and analogue of (2.46)

$$
V^{-}=\sum q_{m} \omega_{m} U_{m}^{-}, \quad\left\|V^{-}\right\|^{2}=\sum\left|q_{m}\right|^{2} \leqslant 1,
$$

the $L_{\infty}$-norm of $R_{\lambda}^{0} V^{-} R_{\lambda}^{0} F$ does not exceed (compare (2.49)) $\frac{1}{\pi^{3}} S$ where

$$
S=\sum_{m, j, l}\left|f_{j} q_{m} \cdot \frac{1}{\mu-j} \cdot \frac{1}{\mu-l} \cdot \frac{\omega_{m}}{m-j-l}\right| .
$$

As in (2.50) we use the identity

$$
\frac{1}{\mu-j} \cdot \frac{1}{\mu-j-l}=\frac{1}{m-l-\mu} \cdot\left(\frac{1}{\mu-j}-\frac{1}{m-j-l}\right)
$$

and later

$$
\frac{1}{\mu-l} \cdot \frac{1}{m-l-\mu}=\frac{1}{m-2 \mu} \cdot\left(\frac{1}{\mu-l}-\frac{1}{m-l-\mu}\right) .
$$

For fixed $m$ (even) and $l$, put

$$
c_{m l}=\sum_{j} f_{j}\left(\frac{1}{\mu-j}-\frac{1}{m-l-j}\right)=c_{m l}^{\prime}+c_{m l}^{\prime \prime} .
$$

The estimates of two parts of this sum $c_{m l}$ are done differently because we need to preserve dependence on $\omega$,

$$
\mu=n+w, \quad|\operatorname{Re} w| \leqslant \frac{1}{2}, \quad w=x+i y,
$$

and specially, on $y=\operatorname{Im} w$, in the final inequality.

For the first term $c^{\prime}$, as in (2.51), (2.52), we have

$$
\left|c_{m l}^{\prime}\right|^{2} \leqslant A^{2}(\mu)=A^{2}(\omega) .
$$

For the second sum $c^{\prime \prime}$ we use the Hilbert transform's boundedness in $l^{2}$, i.e. if

$$
H f=h, \quad h_{a}=\sum_{j} f_{j} \cdot \frac{1}{a-j},
$$

then

$$
h \in l^{2} \quad \text { and } \quad\|h\| \leqslant 3\|f\|
$$

(see [25], Ch. 4, Thm. 9.18 and Exm. 9.23). Therefore,

$$
c_{m l}^{\prime \prime}=h_{m-l}, \quad \text { and } \quad \sum\left|h_{i}\right|^{2} \leqslant 10 .
$$


Now

$$
S=\sum_{m, l}\left|q_{m}\left(c_{m l}^{\prime}+c_{m l}^{\prime \prime}\right) \frac{\omega_{m}}{m-l-\mu} \cdot \frac{1}{\mu-l}\right| .
$$

Put, keeping (2.65) in mind,

$$
D_{m}^{\prime}=\sum_{l}\left|c_{m l}^{\prime}\left(\frac{1}{\mu-l}-\frac{1}{m-l-\mu}\right)\right| .
$$

Then by $(2.68)$

$$
\left|D_{m}^{\prime}\right| \leqslant A(\mu) \cdot B_{m}(\mu)
$$

where

$$
B_{m}(\mu)=\sum_{l}\left|\frac{1}{\mu-l}+\frac{1}{\mu-m+l}\right| .
$$

This function $B_{p}$ will be estimated in Lemma 3.4.

Another part of the sum $S$ in (2.72) comes from

$$
D_{p}^{\prime \prime}=\sum_{l}\left|c_{p l}^{\prime \prime}\left(\frac{1}{\mu-l}+\frac{1}{\mu+l-p}\right)\right|,
$$

and by (2.71) for any $p$

$$
D_{p}^{\prime \prime} \leqslant\left(\sum\left|h_{i}\right|^{2}\right)^{1 / 2}(2 A(\mu)) \leqslant 5 A(\mu) .
$$

Now, with (2.77), (2.74) and (2.65),

$$
S=S^{\prime}+S^{\prime \prime}
$$

with

$$
S^{\prime \prime}=\sum_{p}\left|q_{p} \frac{\omega(p)}{p-2 \mu}\right| D-p^{\prime \prime} \leqslant 5 A(\mu) A_{1}(\mu) .
$$

This is an apologue of (2.53) in the case $\mathcal{H}^{+}$. But to estimate

$$
S^{\prime}=\sum_{p}\left|q_{p} \frac{\omega(p)}{p-2 \mu}\right| D_{p}^{\prime} \leqslant A(\mu)\left(\sum_{p}\left|q_{p}\right|^{2}\right)^{1 / 2}\left(\sum_{p} \frac{\omega^{2}(p)}{|p-2 \mu|^{2}} B_{p}^{2}(\mu)\right)^{1 / 2}
$$

we need good inequalities for $B_{p}(\mu)$ provided by Lemma 3.4 in Sect. 3 and (2.51). They lead to the estimate

$$
S^{\prime} \leqslant A(\mu) 32\left[A_{1}(\mu)+A_{2}(\mu)\right]
$$

where

$$
A_{2}^{2}(\mu)=\sum \frac{\omega^{2}(p)}{|2 n-p+2 w|^{2}} \log ^{2}(2+2|2 n-p|) .
$$

Now we can conclude the case $\mathcal{H}^{-}$. We proved (up to Lemmas 3.5, 3.7c in Section 3) that for $V \in \mathcal{H}^{-}$

$$
\left\|R_{\lambda}^{0} V R_{\lambda}^{0}\right\| \leqslant 40 M A(\mu)\left[\left(\frac{1}{\rho}+1\right) A_{1}(\mu)+A_{2}(\mu)\right] .
$$

Together with (2.53) it proves Proposition 2.4 for Dirichlet $b c$. 
The cases $P e r^{ \pm}$are simpler; they could be split to the component's bc. Technically, the inequalities we need in these cases are essentially the same as in the case $V \in \mathcal{H}^{+}, b c=D i r$. But to avoid any misunderstanding, let us write details, at least in the case $\mathrm{Per}^{-}$.

Case $\mathrm{Per}^{-}$. Eigenvectors for $L_{b c}^{0}$ (see Table, p. 126, line $\mathrm{Per}^{-}$) are pairs of functions

$$
e_{k}^{+}=\sqrt{2}\left(\begin{array}{c}
e^{-i \pi k x} \\
0
\end{array}\right), \quad e_{k}^{-}=\sqrt{2}\left(\begin{array}{c}
0 \\
e^{i \pi k x}
\end{array}\right)
$$

for each odd $k$. For any potential $V$ instead of decomposition (2.38), which fits to Dirichlet $b c$, we have a much simpler decomposition

$$
\mathcal{H}=(H \times\{0\}) \times(\{0\} \times H)
$$

with basis

$$
E_{p}^{+}=\left(\begin{array}{cc}
0 & e^{-i \pi p x} \\
0 & 0
\end{array}\right), \quad E_{p}^{-}=\left(\begin{array}{cc}
0 & 0 \\
e^{i \pi p x} & 0
\end{array}\right), \quad p \text { even }
$$

Any $V=\left(\begin{array}{ll}0 & p \\ q & 0\end{array}\right) \in \mathcal{H}(\Omega)$ has a decomposition $V=P+Q$,

$$
P=\left(\begin{array}{ll}
0 & p \\
0 & 0
\end{array}\right), \quad Q=\left(\begin{array}{ll}
0 & 0 \\
q & 0
\end{array}\right)
$$

and

$$
\begin{gathered}
P=\sum q_{p}^{+} \omega_{p} E_{p}^{+}, \quad Q=\sum q_{p}^{-} \omega_{p} E_{p}^{-}, \\
M^{2}:=\|V \mid \mathcal{H}(\Omega)\|^{2}=\sum\left(\left|q_{p}^{+}\right|^{2}+\left|q_{p}^{-}\right|^{2}\right) .
\end{gathered}
$$

Notice that for any $p$ even, $k \in \mathbb{Z}$

$$
E_{p}^{+} e_{k}^{+}=0, \quad E_{p}^{-} e_{k}^{-}=0, \quad E_{p}^{+} e_{k}^{-}=e_{p-k}^{+}, \quad E_{p}^{-} e_{k}^{+}=e_{p-k}^{-} .
$$

If

$$
F=\sum f-k^{+} e_{k}^{+}+\sum f_{k}^{-} e_{k}^{-} \equiv F^{+}+F^{-}
$$

then $V F=Q F^{+}+P F^{-}$and

$$
R_{\lambda}^{0} V R_{\lambda}^{0} F=R_{\lambda}^{0} Q R_{\lambda}^{0} F^{+}+R_{\lambda}^{0} P R_{\lambda}^{0} F^{-} .
$$

$L_{\infty}$-norms of two terms in (2.92) are estimated in the same way. Let us do details for the first term. Write as in (2.44) or (2.60)

$$
e_{k}^{+} \stackrel{R_{\lambda}^{0}}{\longrightarrow} \frac{1}{\lambda-\pi k} e_{k}^{+} \stackrel{\omega_{p} E_{p}^{-}}{\longrightarrow} \frac{1}{\pi} \frac{\omega_{p}}{\mu-k} e_{p-k}^{-} \stackrel{R-\lambda^{0}}{\longrightarrow} \frac{1}{\pi^{2}} \frac{\omega_{p}}{\mu-k} \cdot \frac{1}{\mu-p+k} e_{p-k}^{-} ;
$$

therefore, with $\left\|e_{p-k}^{-}\right\| \leqslant 2, \forall p, k$

$$
\left\|R_{\lambda}^{0} Q R_{\lambda}^{0} F^{+}\right\|_{\infty} \leqslant \frac{2}{\pi^{2}} \sum_{p}\left|f_{k}^{+} q_{p}^{-} \frac{\omega(p)}{(\mu-k)(\mu-p+k)}\right|
$$

(compare (2.47)). It has already been proven in (2.47)-(2.55) that this quantity does not exceed $M A(\mu) A_{1}(\mu)$.

Of course, a second term in (2.92) can be controlled in the same way. All the decompositions are orthogonal so we even do not need to double the constant to claim

$$
\left\|R_{\lambda}^{0} V R_{\lambda}^{0} F\right\|_{\infty} \leqslant M A(\mu) A_{1}(\mu) .
$$


It completes the proof of Proposition 2.4.

Its part (2.28) incorporates estimates of a function $B \in(2.75)$ given in Lemma 3.4 from the next Section. Of course, we need more information about behavior (growth, decay) of all these functions $A, B, A_{1}, A_{2}$. This is a goal of the next Section to find good inequalities for them.

\section{Inequalities for Sums of Series Which Majorize the Norms of Resolvent Operators}

0. In Section 2 we assumed that $V \in L^{2}$ and $V \in H(\Omega)$. Later in Sections 4-6 we'll explain that our constructionsand results can be carried on for $V \in L^{b}, b>1$ (or $V \in L \log L$, see Sect. 9.1). Keeping this in mind, now we'll consider and estimate more general sums that $A(\mu)$ and $A_{1}(\mu)$ of Sect. 2.In this section we do technical analysis and found good inequalities for the following functions.

$$
A(\sigma ; \mu)=\left(\sum_{k \in \mathbb{Z}} \frac{1}{|k-\mu|^{\sigma}}\right)^{1 / \sigma}, \quad 1<\sigma \leqslant \infty
$$

with understanding that

$$
A(\infty ; \mu)=\max _{k \in \mathbb{Z}} \frac{1}{|k-\mu|} .
$$

Next

$$
A_{1}(\sigma, \omega ; \mu)=\left(\sum_{p \text { even }} \frac{\omega^{\sigma}(p)}{|2 \mu-p|^{\sigma}}\right)^{1 / \sigma}, \quad 1<\sigma \leqslant \infty,
$$

where $\mu=n+\omega$ and

$$
A_{2}(\sigma, \omega ; \mu)=\left(\sum_{m \text { even }} \omega^{\sigma}(m)\left(\frac{\log (2+2|2 n-p|)}{|2 n-p+2 \omega|}\right)^{\sigma}\right)^{1 / \sigma}, \quad 1<\sigma \leqslant \infty .
$$

Finally, for $a$ even, $|\operatorname{Re} w| \leqslant \frac{1}{2}$, define

$$
B_{*}(a, w)=\sum_{k \in \mathbb{Z}}\left|\frac{1}{a-k+w}+\frac{1}{k+w}\right| .
$$

Often, we will omit the sequence $\{\omega\}$ in notations (3.3), (3.4) with understanding that a subscript ' 1 ' or ' 2 ' reminds us on its presence.

Functions in Section 2 came from $V \in L^{2}$, i.e.,

$$
A(\mu)=A(2, \mu), \quad A_{1}(\mu)=A_{1}(2, \mu), \quad \text { etc. }
$$

So inequalities of this Section will imply the mentioned in Sect. 2 inequalities if we put $\sigma=2$.

1. First, we deal with $A(\sigma ; \mu) \in(3.1)$. For any fixed $\sigma$, this is a periodic and even function

$$
A(\sigma ; \mu)=A(\sigma ;-\mu) A(\sigma ; \mu+1)
$$

so let us consider $\mu=\omega=x+i y,|x| \leqslant 1 / 2$. Then

$$
|\mu-k|^{2}=(k-x)^{2}+y^{2},
$$


and if $|k| \geqslant 2$

$$
\frac{1}{2}\left(k^{2}+x^{2}\right) \leqslant(k-x)^{2} \leqslant \frac{3}{2}\left(k^{2}+x^{2}\right) .
$$

Therefore

$$
\sum \frac{1}{|k-\mu|^{\sigma}} \leqslant \frac{3}{\sigma^{\rho}}+\sum_{|k| \geqslant 2} \leqslant \frac{3}{\rho^{\sigma}}+2.2^{\sigma} \cdot \sum,
$$

where

$$
\sum=\sum_{k=2}^{\infty}\left(k^{2}+\rho^{2}\right)^{-\sigma / 2}
$$

Then

$$
\sum<\int_{1}^{\infty} \frac{d k}{k^{\sigma}}=\frac{1}{\sigma-1}
$$

for any $\rho \geqslant 0$, but if $\rho \geqslant 1$ we can do better:

$$
\sum \leqslant \int_{1}^{\infty}\left(k^{2}+\rho^{2}\right)^{-\sigma / 2} d k \leqslant \rho^{1-\sigma} \int_{1 / \rho}^{\infty} \frac{d \xi}{\left(1+\xi^{2}\right)^{\sigma / 2}}<\frac{\sigma}{\sigma-1} \rho^{1-\sigma} .
$$

In any case

$$
\sum<\frac{1}{\sigma-1} \min \left\{1 ; \sigma \rho^{1-\sigma}\right\} \leqslant \frac{\sigma}{\sigma-1} \cdot \frac{2}{\sigma+\rho^{\sigma-1}} .
$$

For $\sigma \geqslant 1, u, v \geqslant 0$ one has

$$
\left(u^{\sigma}+v^{\sigma}\right)^{1 / \sigma} \leqslant u+v \leqslant 2^{1-1 / \sigma}\left(u^{\sigma}+v^{\sigma}\right)^{1 / \sigma} .
$$

By (3.10) and (3.14)

$$
A(\sigma ; \mu) \leqslant \frac{3}{\rho}+4\left(\frac{2 \sigma}{\sigma-1}\right)^{1-\sigma}\left(\sigma+\rho^{\sigma-1}\right)^{-1 / \sigma} .
$$

If $\rho \leqslant 1 / 2, \sigma>1$,

$$
A(\sigma ; \mu) \leqslant \frac{3}{\rho}+C_{1}(\sigma) .
$$

If $\rho \geqslant 1 / 4$ the second term in (3.16) majorizes the first one, up to the factor depending on $\sigma, 1<\sigma \leqslant \infty$, so

$$
A(\sigma ; \mu) \leqslant C_{2}(\sigma) \rho^{1-1 / \sigma},
$$

with

$$
C_{2}(\sigma) \leqslant C_{*} \leqslant \infty
$$

if $\sigma \geqslant 2$, but for $1<\sigma \leqslant 2$ it is continuous, and

$$
(\sigma-1) C_{2}(\sigma) \leqslant C_{*}<\infty .
$$

We can write, after (3.16), these functions $C_{1}(\sigma)$ and $C_{2}(\sigma)$ explicitly but we do not need it and we will not use it. (3.17) or (3.18) will suffice. We have proven 
LEMmA 3.1. If $1<\sigma \leqslant \infty$ then for $C_{3}(\sigma)<\infty, C_{3} \in(3.19) \&(3.20)$,

$$
A(\sigma ; \mu) \leqslant C_{3}(\sigma)\left[\frac{1}{\rho}+\frac{1}{\rho^{1-1 / \sigma}}\right] \text {. }
$$

Corollary 3.2. If $\sigma=2$ then

$$
A(\mu) \equiv A(2 ; \mu) \leqslant \frac{2}{\rho}+\frac{6}{\sqrt{1+\rho}} .
$$

Proof. If we follow (3.10)-(3.16) but put there $\sigma=2$ from the outset we'll come to (3.22), an analogue of (3.16).

2. Now we go to $A-1 \in(3.3)$. Monotonicity of $\{\omega\}, \omega_{p} \searrow 0(p \rightarrow \pm \infty)$, as we assume in (1.7), implies the decay of $A_{1}(\sigma ; \pi n+\pi \omega)$ when $n \rightarrow \pm \infty$ as well.

Lemma 3.3. For $A_{1} \in(3.3)$ and $1 \leqslant r \leqslant n, 2 \leqslant n$ the following inequality holds.

$$
A_{1}(\sigma, \omega ; \mu) \leqslant \frac{\omega(2 n)}{\rho}+C_{4}(\sigma) \frac{\omega(r)}{1+\rho^{1-1 / \sigma}}+\frac{3 r^{1 / \sigma}}{n+\rho} .
$$

In particular, if $r=n$, an inequality (3.29) below holds.

Proof. WLOG we can assume $n>0$ so $n \geqslant 2$. We analyze the sum $\sum_{p \text { even }}$ in the brackets in (3.8). For any $a$,

$$
0<a \leqslant n \leqslant 2 n-2
$$

we write

$$
\sum_{p}=\sum_{|p| \leqslant a}+\sum_{|p|>a} \equiv s_{1}+s_{2} .
$$

With $\omega(p) \leqslant \omega(0)=1$

$$
\begin{aligned}
s_{1} \leqslant & \sum_{|p| \leqslant a}|2 \mu-p|^{-\sigma}=\sum_{|p| \leqslant a}|2 n-p+\omega|^{-\sigma}< \\
& <(2 a+1)|2 n-a+\omega|^{-\sigma} \leqslant \frac{2 a+1}{|n+\omega|^{\sigma}} . l
\end{aligned}
$$

On another side, $\omega(p) \leqslant \omega(a)$ if $|p|>a$ so

$$
s_{2} \leqslant \omega^{\sigma}(a) \sum_{|p|>a} \leqslant \omega^{\sigma}(a) \sum+\frac{\omega^{\sigma}(2 n)}{(2 \rho)^{\sigma}}
$$

(where $\sum$ is defined in (3.11).)

$$
\leqslant \omega^{\sigma}(a) \cdot \frac{\sigma}{\sigma-1} \cdot \frac{2}{\sigma+\rho^{\sigma-1}}+\frac{\omega^{\sigma}(2 n)}{(2 \rho)^{\sigma}}
$$

by (3.12)-(3.13). Again, we use (3.15) and combine (3.25)-(3.27) to conclude that

$$
A_{1}=\left(s_{1}+s_{2}\right)^{1 / \sigma} \leqslant \frac{3 a^{1 / \sigma}}{n+\rho}+\frac{C-3(\sigma) \omega(a)}{1+\rho^{1-1 / \sigma}}+\frac{\omega(2 n)}{\rho} .
$$

In particular, if we coose $a=n$, - it is consistant with (3.24), - we have for $\mu=n+\omega,|\operatorname{Re} \omega| \leqslant 1 / 2, \rho=|\omega|$

$$
A_{1} \equiv A_{1}(\sigma, \omega ; \mu) \leqslant \frac{\omega(2 n)}{\rho}+C-1(\sigma) \frac{\omega(n)}{1+\rho^{1-1 / \sigma}}+\frac{3 n^{1 / \sigma}}{n+\rho} .
$$


3. Functions $B$ and $A_{2}$ came into play in (2.74), (2.75), (2.81). We need to analyze $B$ first, and than it becomes clear that (3.4) is a proper explicit form for $A_{2}$.

As it appear in (2.74)-(2.75),

$$
\begin{aligned}
B_{p}(\mu) & =\sum_{l}\left|\frac{1}{\mu-l}+\frac{1}{\mu-p+l}\right|=\sum_{l}\left|\frac{1}{n-l+w}+\frac{1}{n-p+l+w}\right|= \\
& =\sum_{k}\left|\frac{1}{k+w}+\frac{1}{(2 n-p)-k+w}\right|=B_{*}(2 n-p ; w) .
\end{aligned}
$$

This is our function $B_{*} \in(3.5)$.

LEMma 3.4. For $w,|R e w| \leqslant \frac{1}{2}, \rho=|w|$ and a even we have

$$
B_{*}(a ; w) \leqslant 10+\frac{2}{\rho}+8 \frac{|a|+\rho}{|a|} \log \frac{|a|+1+\rho}{1+\rho}, \quad|a| \geqslant 4,
$$

or

$$
B_{*}(a ; w) \leqslant 16\left(2+\frac{1}{\rho}\right), \quad|a| \leqslant 2 .
$$

REMARK. We'll use its simplified form (3.46).

Proof. As in (3.7) we have

$$
B_{*}(-a ; w)=B_{*}(a ;-w)
$$

so WLOG we can assume $a \geqslant 0$.

Let us consider the case $a \geqslant 4$.

We can split $B_{*}$ into four sums

$$
B_{*}=b_{0}+b_{1}+b_{2}+b_{3}=\sum_{\substack{k=0, \pm 1 \\ k=a, a \pm 1}}+\sum_{2 \leqslant k \leqslant a-2}+\sum_{k \leqslant-2}+\sum_{k \geqslant a+2} .
$$

Then $a \geqslant 4$ and $|w| \leqslant 1 / 2$ imply

$$
b_{0} \leqslant 2\left[\frac{1}{\rho}+\frac{2}{7}+2+\frac{2}{5}+2+\frac{2}{9}\right]<2\left(\frac{1}{\rho}+5\right) .
$$

For $b_{1}$, with $a \geqslant 4$ and $2 \leqslant k \leqslant a-2$ we have $a-k \geqslant 2$ and as in (3.8)-(3.9)

$$
\begin{gathered}
|k+w|^{2} \geqslant \frac{1}{2}\left(k^{2}+\rho^{2}\right), \\
|a-k+w|^{2} \geqslant \frac{1}{2}\left((a-k)^{2}+\rho^{2}\right) .
\end{gathered}
$$

It gives us simplified inequalities

$$
\begin{gathered}
|k+w| \geqslant(k+\rho), \\
|a-k+w| \geqslant \frac{1}{2}((a-k)+\rho) .
\end{gathered}
$$


Therefore, with $a \geqslant 4$,

$$
\begin{gathered}
b_{1} \leqslant \sum_{k=2}^{a-2}\left(\frac{2}{k+\rho}+\frac{2}{a-k+\rho}\right)=4 \sum_{2}^{a-2} \frac{1}{k+\rho} \leqslant \\
\leqslant 4 \int_{1}^{a-2} \frac{d k}{k+\rho}=4 \log \frac{(a-2)+\rho}{1+\rho} .
\end{gathered}
$$

For $b_{2}$, with $k \leqslant-2$ and $a>0 a-k \geqslant 2$, so again we can use (3.38), (3.39) with $|k|$ instead of $k$, i.e.

$$
|k+w| \geqslant \frac{1}{2}(-k+\rho)
$$

Therefore,

$$
\begin{gathered}
b_{2}=\sum_{-\infty}^{-2}\left|\frac{1}{a-k+w}+\frac{1}{k+w}\right|=\sum_{-\infty}^{-2} \frac{|a+2 w|}{|a-k+w| \cdot|k+w|} \leqslant \\
\leqslant|a+2 w| \cdot \sum_{-\infty}^{-2} \frac{4}{(a-k+\rho)(-k+\rho)} \leqslant 4(a+2 \rho) \sum_{2}^{\infty} \frac{1}{a}\left(\frac{1}{k+\rho}-\frac{1}{a+k+\rho}\right)= \\
=4\left(1+2 \frac{\rho}{a}\right) \sum_{2}^{a+1} \frac{1}{k+\rho} \leqslant 4\left(1+\frac{2 \rho}{a}\right) \log \frac{a+1+\rho}{1+\rho} .
\end{gathered}
$$

The sum $b_{3}$ is equal to $b_{2}$ as we can easily see by changing the summation index $k$ to $j=a-k$, so

$$
b_{3}=b_{2} \leqslant(3.42) \text { 's right side. }
$$

Now we can combine (3.35), (3.40), (3.42) and (3.43) and claim (3.31)!

We omit explicit analysis, i.e., the same lines with a few simplifications, which leads to (3.32). Lemma 3.4 is proven.

4. Let us simplify (3.31). If $\rho \leqslant|a|$ then $(|a| \geqslant 4)$

$$
B_{*}(a ; w) \leqslant 10+\frac{2}{\rho}+16 \log \frac{1+2|a|}{1+\rho} .
$$

If $\rho \geqslant|a|($ recall $\log (1+u) \leqslant u$ if $u \geqslant 0)$ then

$$
B_{*}(a ; w) \leqslant 10+\frac{2}{\rho}+8 \cdot \frac{2 \rho}{|a|} \cdot \frac{|a|}{1+\rho}<2\left(\frac{1}{\rho}+13\right) .
$$

In all cases, (3.32) included,

$$
B_{*}(a ; w) \leqslant 16\left(\frac{1}{\rho}+2\right)+16 \log (1+2|a|) .
$$

The second term on the right side justifies our definition (2.82) and (3.4) of the functions $A_{2}(\mu)$ and $A_{2}(\sigma, \omega ; \mu)$.

5. Now we'll give for $A_{2} \in(3.4)$ an analogue of Lemma 3.3, or of its inequalities (3.23) and (3.29).

Lemma 3.5. For $A_{2} \in$ (3.4) and $1 \leqslant a \leqslant n, 2 \leqslant n$ the following inequality holds, $\mu=n+w, \mid$ Re $w \mid \leqslant \frac{1}{2}$ 


$$
A_{2}(\sigma, \omega ; \mu) \leqslant 3 a^{1 / \sigma} \cdot \frac{\log (1+2|n|)}{|n+2 w|}+\omega(a) D(\sigma ; w)
$$

where

$$
D=\left[\sum_{k \text { even }}\left(\frac{\log (1+2|k|)}{|k+2 w|}\right)^{\sigma}\right]^{1 / \sigma}, \quad \mid \text { Re } w \mid \leqslant \frac{1}{2} .
$$

Proof. It follows the same scheme as inequalities (3.24)-(3.27), (3.28) in Lemma 3.3. It is possible because the structure of the sums (compare (3.25)), i.e.,

$$
T(n)=\sum \omega^{\sigma}(\rho) b(2 n-\rho)
$$

where $b$ is an even $l^{1}$-sequence.

Therefore,

$$
T(n)=\sum_{|\rho| \leqslant a}+\sum_{|\rho|>a} \leqslant \sum_{|\rho| \leqslant a} b(2 n-\rho)+\omega^{\sigma}(a) \cdot\|b\|_{1} \leqslant(2 a+1) b(n)+\beta \cdot \omega^{\sigma}(a)
$$

where $\beta=\|b\|_{1}=\sum_{-\infty}^{\infty} b(2 k)$.

We omit further details in explaining inequalities (3.47).

6. Of course, it was an easy part. If we want to apply Lemma 3.5 (and we will do it in Sect. 4-7) we need for $D$ the analogue of inequalities (3.21), (3.22), or Lemma 3.1, Corr. 3.2.

Lemma 3.6. For $1<\sigma \leqslant \infty$ there exists constant $J(\sigma)<\infty$ such that for $D \in(3.48), \rho=|M|$

$$
D \equiv D(\sigma ; \mu) \leqslant J(\sigma) \frac{1+\log (1+\rho)}{1+\rho^{1-1 / \sigma}}, \quad 0<\rho<\infty .
$$

Proof. Notice, like in (3.8), (3.9) that

$$
|1+w|^{2}=(1+x)^{2}+y^{2} \geqslant \frac{1}{5}\left(1+\rho^{2}\right)
$$

so

$$
\frac{1}{|1 \pm w|} \leqslant \frac{\sqrt{5}}{\sqrt{1+\rho^{2}}} \leqslant \frac{\sqrt{10}}{1+\rho} .
$$

We use it and (3.8)-(3.12) in the following sequence of inequalities.

$$
\sum_{k \text { even }}\left(\frac{\log (1+2|k|)}{|k+2 w|}\right)^{\sigma}=2^{-\sigma} \sum_{|q| \geqslant 1}\left(\frac{\log (1+4|q|)}{|q+w|}\right)^{\sigma}=
$$

$$
=2^{-\sigma}\left(\sum_{q= \pm 1}+\sum_{|q| \geqslant 2}\right) \leqslant 2^{-\sigma} \cdot 2\left(\frac{10^{1 / 2} \log 5}{1+\rho}\right)+2^{1-\sigma} \sum_{q=2}^{\infty}\left(\frac{\log (1+4 q)}{\frac{1}{2}(q+\rho)}\right)^{\sigma} \leqslant
$$

$$
\leqslant 2\left(\frac{(5 / 2)^{1 / 2} \log 5}{1+\rho}\right)^{\sigma}+2 \int_{1}^{\infty}\left(\frac{\log (1+x)}{x+\rho}\right)^{\sigma} d x+2 h_{2}(\rho) \equiv h_{0}+2 h_{1}+2 h_{2} .
$$


We specify $h_{2} \equiv h_{2}(\rho)$ later. We use an integral comparision but

$$
\varphi(x)=\frac{\log (1+4 x)}{x+\rho}
$$

and $(\varphi(x))^{\sigma}$, are not always monotone on $[1, \infty)$. Indeed

$$
\varphi^{\prime}(x)=(x+\rho)^{-2}\left[\frac{x+\rho}{x+1 / 4}-\log (1+4 x)\right] .
$$

If $\rho \leqslant \frac{1}{4}$ then $(x+\rho) /(x+1 / 4) \leqslant 1$ but $\log (1+4 x) \geqslant \log 5>1$ so $\varphi^{\prime}(x)<0$ $(x \geqslant 1)$. This is true as long as

$$
\frac{1+\rho}{1+1 / 4}<\log 5, \quad \text { i.e., } \quad \rho<\rho_{*}=\frac{5}{4} \log 5-1 .
$$

(For curiosity, let us notice that $\rho_{*}=1.011797 \ldots$, i.e.

$$
\rho_{*}>1 \text {.) }
$$

Therefore, we do not need an extra term $h_{2}$ when the sum is majorized by the integral $h_{1}$, i.e., we can choose

$$
h_{2}(\rho)=0 \quad \text { if } \quad 0<\rho \leqslant \rho_{*} .
$$

If however $\rho>\rho_{*}$ there is ONE and ONLY ONE root $x^{*}>1$

$$
\varphi^{\prime}\left(x^{*}\right)=0, \quad \text { and } \quad \begin{aligned}
& \varphi^{\prime}(x)>0, \quad 1 \leqslant x<x^{*} \\
& \varphi^{\prime}(x)<0, \quad x^{*}<x<\infty
\end{aligned}
$$

as it follows from (3.58). Explicitly, an equation for $x^{*}$ is

$$
\rho=1 / 4+\left(x^{*}+1 / 4\right)\left[\log \left(1+4 x^{*}\right)-1\right] .
$$

(3.55)-(3.56) will be correct if we'll choose $h_{2}(\rho) \geqslant\left(\max _{x \geqslant 1} \varphi(x)\right)^{\sigma}$ for $\rho>\rho_{*}$.

But for any $\rho$

$$
\varphi(x)=\frac{\log (1+4 x)}{x+\rho} \leqslant \frac{\log (1+4 \rho)}{\rho} \quad \text { if } 0 \leqslant x \leqslant \rho .
$$

If $x \geqslant \rho$ we use another inequality

$$
\varphi(x) \leqslant \frac{\log (1+4 x)}{x} \leqslant \frac{\log (1+4 \rho)}{\rho}, \quad x \geqslant \rho .
$$

It is correct because for $x \geqslant 1$

$$
\left(\frac{1}{x} \log (1+4 x)\right)^{\prime}=x^{-2}\left[\frac{x}{x+1 / 4}-\log (1+4 x)\right]<x^{-2}[1-\log 5]<0,
$$

and $\max _{x \geqslant \rho} \varphi(x)=\varphi(\rho)$.

Finally, we choose

$$
h_{2}(\rho)= \begin{cases}\left(\frac{\log (1+4 \rho)}{\rho}\right)^{\sigma}, & \rho \geqslant \rho_{*} \\ 0, & 0<\rho \leqslant \rho_{*} .\end{cases}
$$

Then (3.55)-(3.56) holds.

7. Now, for any $\rho \geqslant 0$

$$
h_{1} \leqslant I_{1}(\sigma)=\int_{1}^{\infty}\left(\frac{\log (1+4 x)}{x}\right)^{\sigma} d x<\infty \quad(\sigma>1) .
$$


We need a better estimate for large $\rho$.

$$
\begin{aligned}
& h_{1}=\int_{1}^{\infty}\left(\frac{\log (1+4 x)}{x+\rho}\right)^{\sigma} d x<\rho^{1-\sigma} \int_{0}^{\infty}\left(\frac{\log (1+4 \rho \xi)}{1+\xi}\right)^{\sigma} d \xi \leqslant \\
& \leqslant 2^{\sigma-1} \rho^{1-\sigma} \int_{0}^{\infty}\left[\left(\frac{\log (1+4 \xi)}{1+\xi}\right)^{\sigma}+\left(\frac{\log (1+\rho)}{1+\xi}\right)^{\sigma}\right] d \xi .
\end{aligned}
$$

We used (3.15) for the second inequality, and an obvious inequality

$$
1+4 \rho \xi \leqslant(1+4 \xi)(1+\rho) .
$$

Therefore,

$$
h_{1} \leqslant\left[I_{2}(\sigma)+I_{3}(\sigma)(\log (1+\rho))^{\sigma}\right] \cdot \rho^{1-\sigma}
$$

where

$$
\begin{gathered}
I_{3}(\sigma)=\frac{2^{\sigma-1}}{\sigma-1}, \\
I_{2}(\sigma)=2^{\sigma-1} \int_{0}^{\infty}\left(\frac{\log (1+4 \xi)}{1+\xi}\right)^{\sigma} d \xi .
\end{gathered}
$$

For curiosity, we can notice that

$$
I_{2}(\sigma)<8^{\sigma-1} \int_{1}^{\infty}\left(\frac{\log y}{y}\right)^{\sigma} d y=8^{\sigma-1}\left(\frac{1}{\sigma-1}\right)^{\sigma+1} \Gamma(\sigma+1) .
$$

By Young inequality (3.15) and (3.71)

$$
h_{1}^{1 / \sigma} \leqslant\left[i_{2}^{1 / \sigma}(\sigma)+I_{3}^{1 / \sigma}(\sigma)(\log (1+\rho))\right] \rho^{1 / \sigma-1}, \quad \forall \rho,
$$

and by (3.68)

$$
h_{1}^{1 / \sigma} \leqslant I_{1}^{1 / \sigma}(\sigma), \quad \forall \rho .
$$

Although specific form of $I_{j}(\sigma), j=1,2,3$, is not essential.

It is important to point out that functions $\left(I_{j}(\sigma)\right)^{1 / \sigma}, j=1,2,3$, are bounded at $+\infty$. For example

$$
I_{3}^{1 / \sigma}=2 \cdot \frac{2^{-1 / \sigma}}{(\sigma-1)^{1 / \sigma}} \rightarrow 2 \quad(\sigma \rightarrow \infty)
$$

and by (3.74)

$$
I_{2}^{1 / \sigma}(\sigma) \leqslant 8^{1-1 / \sigma} \frac{\Gamma^{1 / \sigma}(\sigma+1)}{(\sigma-1)^{1+1 / \sigma}} \rightarrow \frac{8}{e} \quad(\sigma \rightarrow \infty) .
$$

We can choose min of the right sides in (3.75) or (3.76) and with proper choice of a continuous $J_{1}(\sigma), 1<\sigma<\infty, \limsup _{\sigma \rightarrow \infty} J_{1}(\sigma)<\infty$.

$$
h_{1}^{1 / \sigma} \leqslant J_{1}(\sigma) \cdot \frac{1+\log (1+\rho)}{1+\rho^{1-1 / \sigma}} .
$$

An inequality (3.67) and an observation (3.60) imply that

$$
h_{2}^{1 / \sigma} \leqslant 2 \frac{\log (1+4 \rho)}{1+\rho}, \quad \forall \rho
$$


and

$$
h_{2}^{1 / \sigma} \leqslant 3 \frac{1+\log (1+\rho)}{1+\rho^{1-1 / \sigma}}, \quad \forall \rho, \forall \sigma .
$$

The case $h_{0}$ is easy; by $(3.55)$

$$
h_{0}^{1 / \sigma} \leqslant \frac{7}{1+\rho}<\frac{14}{1+\rho^{1-1 / \sigma}} .
$$

Now we collect inequalities (3.82), (3.79) and (3.81) into (3.55) and use Young inequality for three terms

$$
\left(u^{\sigma}+v^{\sigma}+w^{\sigma}\right)^{1 / \sigma} \leqslant(u+v+w), \quad \sigma \geqslant 1, \quad u, v, w \geqslant 0 .
$$

This leads to our main inequality

$$
D \leqslant 2\left(h_{0}^{1 / \sigma}+h_{1}^{1 / \sigma}+h_{2}^{1 / \sigma}\right) \leqslant J(\sigma) \cdot \frac{1+\log (1+\rho)}{1+\rho^{1-1 / \sigma}}
$$

with

$$
J(\sigma)=J_{1}(\sigma)+17, \quad J_{1} \in(3.79) .
$$

Lemma 3.6 is proven.

REMARK. Looking more carefully into $I_{1} \in(3.68), I_{2} \in(3.72) \&(3.74)$ we can explain that

$$
\sup _{1<\sigma \leqslant 2}(\sigma-1)^{2} J(\sigma)<\infty .
$$

8. We will use Lemma 3.5 in Section 6.7 with different choices of $a, 1 \leqslant a \leqslant$ $n$, but let us write its specification with $a=n$ and estimates for $D$ in (3.51), Lemma 3.6.

Lemma 3.7. For $A_{2} \in(3.4)$ and $|n| \geqslant 2, \mu=n+w, \mid$ Re $w\left|<\frac{1}{2}, \rho=\right| w \mid$ the following inequality holds

$$
A_{2}(\sigma, \omega ; \mu) \leqslant 3 \frac{|n|^{1 / \sigma} \log (1+2|n|)}{|n|+\rho}+\omega(|n|) J(\sigma) \frac{1+\log (1+\rho)}{1+\rho^{1-1 / \rho}}
$$

where $J(\sigma)$ defined in Lemma 3.6

It is interesting to compare this inequality with (3.29) for $A_{1}$ where the term $1 / \rho$ in (3.86) and (3.47) has the same power $1 / \sigma-1$.

\section{Trapping the Spectrum}

0 . Now, with the estimates of Section 3, we are ready to construct contours $\Gamma$ containing $\sigma\left(L_{b c}^{0}\right)$ (see Table, p. 126) and

$(*)$ such that $\Gamma$ cannot be crossed - either from inside or from outside - by eigenvalues of

$$
L_{b c}, \quad L(t)=L^{0}+t V
$$

when $t, 0 \leqslant t \leqslant 1$, changes from 0 to 1 .

$\Gamma$ will have the following structure.

For each $n,|n|>N, N$ to be chosen later, and $\delta, 0<\delta<\pi$, we define

$$
D(n ; \delta)=\{z|| z-\pi n \mid \leqslant \delta\}
$$


and

$$
C(n ; \delta)=\partial D(n ; \delta)=\{z|| z-\pi n \mid=\delta\} .
$$

With $N$ chosen, we construct a rectangle

$$
\begin{gathered}
R(X ; Y)=\left\{z \in \mathbb{C}^{1}|| \operatorname{Re} z|\leqslant X,| \operatorname{Im} z \mid \leqslant Y\right\}, \\
X=\pi N+\frac{\pi}{2} .
\end{gathered}
$$

Then

$$
\Gamma=\partial R \cup \bigcup_{|n|>N} C\left(n ; \delta_{n}\right) .
$$

Its parameters $N, Y$ and $\left\{\delta_{n}\right\}, \delta_{n} \searrow 0$, are to be choosen to guarantee $\left(^{*}\right)$.

In this Section we'll write all the detail for the case $V \in L^{2}$. All necessary adjustements for more general case $V \in L^{b}, 1<b$, are explained in the conclusion of this Section although technical inequalities come from Section 3 and Section 5.

In this Section, at least until Subsection 4.7, we use functions $A, A_{1}, A_{2}$ only in the case $\sigma=2$ so the index is omitted in the same way as in Section 2.

1. As before, see (2.1), (2.2), (2.21) we (want to) use the representations

$$
R_{\lambda}=(\lambda-L)^{-1}=R_{\lambda}^{0}\left(1-V R_{\lambda}^{0}\right)^{-1}
$$

or

$$
R_{\lambda}=R_{\lambda}^{0}\left(1+V R_{\lambda}^{0}\right)\left(1-V R_{\lambda}^{0} V R_{\lambda}^{0}\right)^{-1} .
$$

$R_{\lambda}^{0}$ is well-defined for $\lambda \notin \sigma\left(L_{b c}^{0}\right)$, certainly for $\lambda \neq \pi n, n \in \mathbb{Z}$, and therefore on $\Gamma \in(4.6)$.

(4.7) defines $R_{\lambda}$ if

$$
\left\|V R_{\lambda}^{0}\right\| \leqslant \frac{1}{2}
$$

and (4.8) gives good representation of $R_{\lambda}$ if

$$
\left\|V R_{\lambda}^{0} V R_{\lambda}^{0}\right\| \leqslant \frac{1}{2} .
$$

We proved in Proposition 2.3, Section 2 (see (2.19)) that

$$
\left\|V R_{\lambda}^{0}\right\| \leqslant \frac{15}{\rho^{1 / 2}}\|V\|_{2} \leqslant \frac{30 m}{|\operatorname{Im} \lambda|^{1 / 2}} .
$$

Therefore (4.9) holds if

$$
|\operatorname{Im} \lambda| \geqslant(60 m)^{2}, \quad m \geqslant\|V\|_{2},
$$

and (4.9) holds on horizontal intervals

$$
\left\{z \in \mathbb{C}^{1}|| \operatorname{Re} z \mid \leqslant X, \operatorname{Im} z= \pm Y\right\} \subset \partial R \subset \Gamma
$$

for any $X$ if $Y \geqslant(60 m)^{2}$.

It is nice to observe that the height of the rectangle $R \in(4.4)$ is controlled by $L^{2}$-norm of the potential $V$, not by $\|V \mid H(\Omega)\|$.

2. On other pieces of $\Gamma$, we need (4.10) and all the inequalities from Sections 3 and 2 .

First, let us do necessary evaluations for $\mathrm{Per}^{ \pm}$, or $\mathrm{Dir}$ when the potential $V \in \mathcal{H}^{+}$(see Section 2, (2.34), and case $\mathcal{H}^{+}$there).By Proposition 2.4, in all these cases the inequalitites $(2.27)$, or (2.53), (2.95) holds, i.e. 


$$
\left\|R_{\lambda}^{0} V R_{\lambda}^{0}\right\|_{2 \rightarrow \infty} \leqslant 2 M A(\mu) A_{1}(n, w)
$$

where $\lambda=\pi \mu, \mu=n+w, \mid$ Re $w \mid \leqslant 1 / 2$, and $A \in(3.1), A_{1} \in(3.2), M \geqslant\|V \mid H(\Omega)\|$.

If

$$
\lambda=(n+x) \pi+i v, \quad|x| \leqslant \frac{1}{2}
$$

then by (3.6), or Lemma $2.2,(2.17), \rho \geqslant \frac{1}{2}$, and

$$
A^{2}(\mu) \leqslant \frac{3}{\rho^{2}}+\frac{10}{1+\rho}
$$

and

$$
A(\mu)<\frac{2}{\rho}+\frac{4}{\sqrt{1+\rho}}
$$

By Lemma 3.5, (3.3),

$$
A_{1}^{2}(n, \omega) \leqslant 3\left[\omega^{2}(n)\left(\frac{1}{\rho^{2}}+\frac{1}{1+\rho}\right)+\frac{|n|}{n^{2}+\rho^{2}}\right]
$$

and

$$
A_{1}(n, \omega) \leqslant 2\left[\omega(n)\left(\frac{1}{\rho}+\frac{1}{\sqrt{1+\rho}}\right)+\frac{|n|^{1 / 2}}{\sqrt{n^{2}+\rho^{2}}}\right]
$$

If

$$
\lambda \in \partial R, \quad \lambda= \pm\left(N+\frac{1}{2} \pi+i v\right), \quad|v| \leqslant Y,
$$

then $\rho=\frac{1}{2}$, and

$$
\begin{gathered}
A(\mu)<4+4=8, \\
A_{1}(n, \omega) \leqslant 6\left(\omega(n)+\frac{1}{\sqrt{n}}\right) .
\end{gathered}
$$

Therefore for $\lambda \in(4.19)$

$$
\left\|R_{\lambda}^{0} V R_{\lambda}^{0}\right\|_{2 \rightarrow \infty} \leqslant 100 M\left(\omega(n)+\frac{1}{\sqrt{|n|}}\right) .
$$

Let us define

$$
N_{1}(\varepsilon)=\min \left\{k: \omega(k)+k^{-1 / 2} \leqslant \varepsilon\right\} .
$$

By $(1.7) \omega(k)=1 / \Omega(k) \rightarrow 0$ monotonically so $N_{1}$ is well-defined and

$$
N_{1}(\varepsilon) \nearrow \infty \text { when } \varepsilon \searrow 0 .
$$

(4.10) holds if

$$
\|V\|_{2} \cdot\left\|R_{\lambda}^{0} V R_{\lambda}^{0}\right\|_{2 \rightarrow \infty} \leqslant 100 m M\left(\omega(n)+\frac{1}{\sqrt{n}}\right)<\frac{1}{2},
$$

or (4.10) holds if

$$
|n| \geqslant \widetilde{N}_{1}(m M)=N_{1}\left(\frac{1}{200 m M}\right)
$$




$$
\widetilde{N}_{1}(x)=N_{1}\left(\frac{1}{200 x}\right) .
$$

3. If we did not care to choose $\delta_{n} \searrow 0$ but take $\delta_{n} \equiv \rho>0,|n| \geqslant N$, we could take

$$
N=\widetilde{N}_{1}\left(\rho^{-2} m M\right),
$$

and the inequalities of the prevoius subsection would guarantee that (4.10) held on $C(n, \delta),|n| \geqslant N, N \in(4.28)$.

If however we want the sequence of radiuses $\delta_{n} \searrow 0$ decaying to zero we need to compensate such a decay in inequalities (4.16) and (4.18). If $\rho$ is small, let us say $\rho \leqslant \frac{1}{2}$, then by $(4.14)-(4.18)$

$$
\begin{aligned}
&\left\|V R_{\lambda}^{0} V R_{\lambda}^{0}\right\| \leqslant m \cdot 2 M \cdot 2\left(\frac{1}{\rho}+2\right) \cdot 2\left(\omega(n)\left(\frac{1}{\rho}+1\right)+\frac{1}{\sqrt{|n|}}\right) \leqslant \\
& \leqslant 30 m M\left(\frac{\omega(n)}{\rho^{2}}+\frac{|n|^{-1 / 2}}{\rho}\right) .
\end{aligned}
$$

If

$$
\rho=\delta_{n}=\left(\omega^{1 / 2}(n)+|n|^{-1 / 2}\right)^{\gamma}, \quad 0<\gamma<1,
$$

then the factor on the right side of (4.30)

$$
\frac{\omega(n)}{\left(\omega^{1 / 2}(n)+|n|^{-1 / 2}\right)^{2 \gamma}}+\frac{|n|^{-1 / 2}}{\delta_{n}} \leqslant \omega^{1-\gamma}(n)+|n|^{-1 / 2(1-\gamma)} \rightarrow 0 .
$$

Let us define (by choosing $\gamma=7 / 8$ )

$$
N_{2}(\varepsilon)=\min \left\{k: \omega^{1 / 8}(k)+k^{-1 / 16} \leqslant \varepsilon\right\} .
$$

Then (4.29)-(4.32) guarantee that for $n,|n| \geqslant \widetilde{N}_{2}(m M)$, where

$$
\widetilde{N}_{2}(x)=N_{2}\left(\frac{1}{60 x}\right) \text {, }
$$

our dream-inequality (4.10) holds.

With $\omega(k) \leqslant 1,(4.23)$ and (4.33) imply that

$$
N_{2}(\varepsilon) \geqslant N_{1}\left(\frac{\varepsilon}{4}\right) \text {. }
$$

Now we define $\Gamma \in(4.6)$ by choosing

$$
N=\widetilde{N}_{2}(m M), \quad \delta_{n}=\left(\omega^{1 / 2}(n)+\frac{1}{\sqrt{n}}\right)^{7 / 8} .
$$

4. With (4.9) on the part (4.13) of $\Gamma$ and (4.10) on $\Gamma \backslash$ (4.13), we succeeded to explain that $R_{\lambda}=\left(\lambda-L_{b c}\right)^{-1}$ is well-defined on $\Gamma$. Therefore, we have proven the following statement.

Theorem 4.1. Let us consider $L_{b c}, b c=P e r^{ \pm}$, or $b c=$ Dir but $V \in \mathcal{H}^{+}$, and assume that

$$
\|V\|_{2} \leqslant m, \quad\|V \mid H(\Omega)\| \leqslant M .
$$

Define contour $\Gamma \in(4.4)-(4.6)$ by choosing

$$
Y=(60 m)^{2}, \quad X=\pi N+\frac{\pi}{2},
$$


and $N$ and $\left\{\delta_{n}\right\}$ being defined by (4.36). Then

$$
\sigma\left(L_{b c}\right) \subset R(X ; Y) \cup \bigcup_{|n|>N} D\left(n ; \delta_{n}\right) .
$$

5. The twin-statement for Dir, $\mathcal{H}^{-}$case, can be proven along the same lines but with proper adjustment of the inequality (4.14). According to Proposition 4 - see $(2.27),(2.28)$ and in particular $(2.79)$ - in $b c=D i r$ case we have instead of (4.14) the following inequality

$$
\nu=\left\|R_{\lambda}^{0} V R_{\lambda}^{0}\right\|_{2 \rightarrow \infty} \leqslant 40 M A(\mu)\left[\left(\frac{1}{\rho}+1\right) A_{1}(\mu)+A_{2}(\mu)\right] .
$$

Now, together with (4.16), (4.18) we need to use (3.42). This inequality implies for $\rho \leqslant 1 / 2$

$$
A_{2}(\mu) \leqslant 5\left[2 \omega(\mu)+\frac{\log (1+2|n|)}{n^{1 / 2}}\right] .
$$

If we use (4.16), (4.18) and (4.41) in (4.40) we get

$$
\begin{gathered}
\nu=\left\|R_{\lambda}^{0} V R_{\lambda}^{0}\right\|_{2 \rightarrow \infty} \leqslant 40 M \cdot 2\left(2+\frac{1}{\rho}\right) \times \\
{\left[\left(\frac{1}{\rho}+1\right)\left(2 \omega(n)\left(\frac{1}{\rho}+1\right)+\frac{1}{\sqrt{n}}\right)+10 \omega(n)+5 \frac{\log (1+2|n|)}{n^{1 / 2}}\right] \leqslant} \\
\leqslant 40 M \cdot 6\left[\frac{8}{\rho^{3}}+\frac{2}{\rho^{2}}|n|^{-1 / 2}+\frac{10}{\rho}\left(\omega(n)+\frac{\log (1+2|n|)}{n^{1 / 2}}\right)\right] .
\end{gathered}
$$

If $\rho=1 / 2$

$$
\nu \leqslant C^{*}\left[\omega(n)+\frac{\log (1+2|n|)}{|n|^{1 / 2}}\right],
$$

$C^{*}$ being an absolute constant.

Let us define

$$
N_{1}(\varepsilon)=\min \left\{k>0 \mid \omega(k)+\frac{\log (1+k)}{k^{1 / 2}}<\varepsilon\right\}
$$

and

$$
\widetilde{N}_{1}(\varepsilon)=N_{1}\left(\frac{1}{2 C^{*} x}\right)
$$

Then for

$$
N \geqslant \widetilde{N}_{1}(m M)
$$

and any potential $V$ with

$$
\|V\|_{2} \leqslant m, \quad\|V \mid H(\Omega)\| \leqslant M
$$

The inequality (4.10), $b c=$ Dir, holds on the vertical sides of a rectangle $R \in$ $(4.4)+(4.5)$.

Next, like in (4.31) we'll choose

$$
\rho=\delta_{n}=\left(\omega^{1 / 3}(n)+n^{-1 / 4}\right)^{\gamma}, \quad 0<\gamma<1 .
$$

Then on $\partial D\left(n ; \delta_{n}\right)=C_{n}(4.42)$ implies

$$
\nu \leqslant M C^{* *}\left[\omega^{1-\gamma}+n^{-1 / 2(1-\gamma)}\right] .
$$


Again, we can put $\gamma=7 / 8$ and take the same definition of $N_{2}(\varepsilon)$ as in (4.33) but

$$
\widetilde{N}_{2}(x)=N_{2}\left(\frac{1}{2 C^{* *} x}\right) .
$$

For $n,|n| \geqslant \widetilde{N}_{2}(m M),(4.48)$ and (4.47) guarantee that on $C_{n}$ (4.10) holde, i.e.

$$
\left\|V R_{\lambda}^{0} V R_{\lambda}^{0}\right\| \leqslant \frac{1}{2}
$$

Therefore, (4.8) tells us that $R_{\lambda}$ is well-defined on $\bigcup_{|n|>N} C_{n}$. Moreover, if $\lambda=n \pi+w, \mid$ Re $w \mid \leqslant \pi / 2$,

$$
\left\|R_{\lambda}\right\| \leqslant\left\|R_{\lambda}^{0}\right\|\left(1+m\left\|R_{\lambda}^{0}\right\|_{2 \rightarrow \infty}\right) \cdot 2 \leqslant \frac{2}{|w|}(1+m A(\mu)) .
$$

We have proven the following

Theorem 4.2. Let $b c=$ Dir, and $V \in$ (4.37). Define contour $\Gamma \in(4.4)-(4.6)$ by choosing $Y=(60 m)^{2}, X=\pi N+\pi / 1$ with $N=\widetilde{N}_{2}(m M), \widetilde{N}_{2} \in(4.49)$, and $\left\{\delta_{n}\right\} \in(4.47)$. Then

$$
\sigma\left(L_{b c}\right) \subset R(X, Y) \cup \bigcup_{|n|>N} D\left(n ; \delta_{n}\right) .
$$

6. Let us notice that $\Gamma$, the contour in Theorem 1 or 2 , depends on $m$ and $M$, and the conclusion (4.39) or (4.51) is valid for ANY potential $V \in(4.37)$. If we consider a family

$$
L_{b c}=L(t)=L_{b c}^{0}+t V, \quad 0 \leqslant t \leqslant 1,
$$

then of course all of its potentials $t V, 0 \leqslant t \leqslant 1$, are in the ball (4.37). So

$$
R(\lambda ; t)=\left(\lambda-L_{b c}^{0}-t V\right)^{-1}, \quad 0 \leqslant t \leqslant 1
$$

is a family of operator-valued functions and for each piece $\gamma$ of the contour $\Gamma$

$$
\gamma=\partial R \quad \text { or } \quad \gamma=C_{n}, \quad|n|>N
$$

it depends continuously on $t$ in the norm

$$
\|A\|_{\gamma}=\max \{\|A(\lambda)\|: \lambda \in \gamma\}
$$

on the space of the continuous operator-valued functions on $\gamma$.

Therefore, projectors

$$
P_{*}(t)=\frac{1}{2 \pi i} \int_{\partial R}\left(\lambda-L_{b c}^{0}-t V\right)^{-1} d \lambda
$$

and

$$
P_{n}(t)=\frac{1}{2 \pi i} \int_{C_{n}} R(\lambda ; t) d \lambda
$$

depends continuously on $t, 0 \leqslant t \leqslant 1$, as well. 
If $t=0$ these projectors are orthogonal, and we show their values explicitly by Table. Certainly, for each $\gamma \in(4.54) \operatorname{dim} P_{\gamma}(0)<\infty$ and by M. KreinM. Krasnoselski-D. Milman Theorem (see M. Naimark [20], Sect. 14.10, Thm. 11) $\operatorname{dim} P_{*}(t), \operatorname{dim} P_{n}(t)$ are constant functions, and if $P_{\gamma}=P_{\gamma}(1)$

$$
\begin{aligned}
& \operatorname{dim} P_{*}=\operatorname{dim} P_{*}(0), \\
& \operatorname{dim} P_{n}=\operatorname{dim} P_{n}(0) .
\end{aligned}
$$

It means that we have proven the following complement to Lemma 3.7 and Theorems 4.1 and 4.2 .

Proposition 4.3. Under assumptions and notations of Lemma 3.7 and Theorem 4.1, dimensions of Riecz spaces Im $P_{\gamma}$ are given by the following Table 4.2.

\begin{tabular}{l|l|ccc}
\multicolumn{2}{c}{$b c$} & Per $^{+}$ & Per $^{-}$ & Dir \\
\hline \multirow{2}{*}{$\operatorname{dim} P_{*}$} & $N$ even & $2 N+1$ & $2 N$ & $2 N+1$ \\
& $N$ odd & $2 N-1$ & $2 N+2$ & $2 N+1$ \\
\hline $\operatorname{dim} P_{n}$ & $n$ even & 2 & 0 & 1 \\
$|n|>N$ & $n$ odd & 0 & 2 & 1 \\
\hline
\end{tabular}

7. So far in this Section we've done all the estimates and proofs in the case of $L^{2}$-potential $V$ and in $L^{2}$-space. Now we'll assume that $V$ is in $L^{b}, 1<b \leqslant 2, V \in$ $K(m, M) \in(5.13)$ and extend this Section's results. We use technical inequalities from the next Section 5 .

As in Section 4.1 we use two representations (4.7) and (4.8) of the resolvent $R_{\lambda}$. But each element there should be well-defined or understood.

First, let us use (4.7) to define $R_{\lambda}$ in $L^{d}, b \leqslant d \leqslant \infty$. The formula

$$
R_{\lambda}=R_{\lambda}^{0}\left(I-V R_{\lambda}^{0}\right)^{-1}
$$

gives the factorization

$$
L^{d} \stackrel{I}{\rightarrow} L^{b} \stackrel{\left(I-V R_{\lambda}^{0}\right)^{-1}}{\longrightarrow} L^{b} \stackrel{R_{\lambda}^{0}}{\longrightarrow} L^{d}
$$

if $d \geqslant 2$, or the last step is split into

$$
L^{b} \stackrel{R_{\lambda}^{0}}{\longrightarrow} L^{2} \stackrel{I}{\rightarrow} L^{d}
$$

if $d<2$, i.e., we do not hope to have better estimates that coming from an inequality

$$
\left\|T: L^{b} \rightarrow L^{d}\right\| \leqslant\left\|T: L^{b} \rightarrow L^{2}\right\| \quad \text { if } d \leqslant 2 .
$$

The operator $\left(I-V R_{\lambda}^{0}\right)^{-1}$ is defined in $L^{b}$ if

$$
\left\|V R_{\lambda}^{0}: L^{b} \rightarrow L^{b}\right\|<1 .
$$

$$
\left\|V R_{\lambda}^{0}: L^{b} \rightarrow L^{b}\right\| \leqslant\|\nu\| \cdot\left\|R_{\lambda}^{0}: L^{b} \rightarrow L^{\infty}\right\| \leqslant m A(b ; \mu) \leqslant c(b) m\left(\frac{1}{\rho}+\frac{1}{\rho^{1-1 / b}}\right) .
$$

The right side is $\leqslant 1 / 2$ if $\rho$ is large enough; more precisely, if

$$
\rho>(2 c(b) m)^{\frac{b}{b-1}} .
$$


The right side of $(4.65)$ is $\leqslant 1 / 2$, and

$$
\left\|V R_{\lambda}^{0}: L^{b} \rightarrow L^{b}\right\| \leqslant \frac{1}{2} \quad \text { if } \quad \lambda \in(4.66)
$$

and

$$
\left(V R_{\lambda}^{0}\right)^{-1} \text { is defined in } L^{b} \text { and its norm } \leqslant 2 .
$$

But we have to return to $L^{d}$.

The last step in (4.61) is $R_{\lambda}^{0}: L^{b} \rightarrow L^{d}$.

According to Lemma 5.2, inequality (5.21), and final remark (4.63),

$$
\left\|R_{\lambda}^{0}: L^{b} \rightarrow L^{d}\right\| \leqslant A(\sigma ; \mu)
$$

where

$$
\frac{1}{\sigma}= \begin{cases}\frac{1}{b}-\frac{1}{d} & \text { if } d \geqslant 2, \\ \frac{1}{b}-\frac{1}{2} & \text { if } d<2 .\end{cases}
$$

In any case, $1<\sigma \leqslant \infty$ and $A(\sigma ; \mu)$ is well-defined and finite, $\mu \notin \mathbb{Z}$.

It leads us to an analogue of Lemma 2.2.

Lemma 4.4. If (4.66) holds $\lambda$ is a regular point in the space $L^{d}$, and

$$
\left\|R_{\lambda}\right\| \leqslant 2\left\|R_{\lambda}^{0}: L^{b} \rightarrow L^{d}\right\| \leqslant 2 A(\sigma ; \mu), \quad \sigma \in(4.70) .
$$

The spectrum $\sigma\left(L_{b c} ; L^{d}\right), b \leqslant d \leqslant \infty$, lies in the strip

$$
\left\{z \in \mathbb{C}^{1}:|\operatorname{Im} z| \leqslant(\widetilde{C}(b) m)^{\frac{1}{b-1}}\right\} .
$$

8. As in Subsections 4.1-4.2, to analyze $R_{\lambda}$ in the strip (4.72) we use representation (4.8). Norms of factors $R_{\lambda}^{0}$ and $I+V R_{\lambda}^{0}$ have already been estimated in (4.69) and (4.65) soto make the third factor $\left(I-K^{2}\right)^{-1}=\left(I-V R_{\lambda}^{0} V R_{\lambda}^{0}\right)^{-1}$ be well-defined as an operator in $L^{b}, 1<b$, it will suffice to guarantee an inequality

$$
\left\|V R_{\lambda}^{0} V R_{\lambda}^{0}: L^{b} \rightarrow L^{b}\right\| \leqslant \frac{1}{2}
$$

Or

$$
w^{*}=\left\|R_{\lambda}^{0} V R_{\lambda}^{0}: L^{b} \rightarrow L^{\infty}\right\| \leqslant \frac{1}{2 m} .
$$

Proposition 5.4 gives estimates on this norm $w^{*}$. By (5.31) and (5.34)

$$
w^{*} \leqslant K_{4}(b) A(b ; \mu) A_{1}(b ; \mu)
$$

if $b c=P e r^{ \pm}, D i r \mathcal{H}^{+}$, and

$$
w^{*} \leqslant K_{5}(b) A(b ; \mu) A_{2}(b ; \mu)
$$

if $b c=$ Dir.

Therefore if the right side in (4.75) (or (4.76) for $b c=D i r$ ) is less than $1 / 2 m$ the condition (4.74) holds and the third factor $\left(I-K^{2}\right)^{-1}$ is well-defined and its norm in $L^{b}$ does not exceed 2 . To be more specific about regular $\lambda$ in the strip, or for small $\rho$, we need Lemma 3.3, inequality (3.29) for $A_{1}$ and Lemma 3.7, inequality (3.86) for $A_{2}$. They imply that in the case (4.75)

$$
w^{*} \leqslant \frac{C}{\rho}\left[\frac{\omega(2 n)}{\rho}+C_{4}(b) \omega(b)+3 n^{-(1-1 / b)}\right]
$$


and in the case (4.76)

$$
w^{*} \leqslant \frac{C_{5} M}{\rho}\left[\frac{\omega(2 n)}{\rho}+\omega(n)+n^{-(1-1 / b)} \log (1+2 n)\right] .
$$

The factor $1 / \rho$ comes from the estimate of the function $A$ given by inequality (3.47).

For $\rho \geqslant 1 / 4$ and adjusting a constant factor $C$ we choose now $N=\widetilde{N}(C m M)$ in such a way that for all $n,|n| \geqslant N$,

$$
\left\|V R_{\lambda}^{0} V R_{\lambda}^{0}: L^{b} \rightarrow L^{b}\right\| \leqslant \frac{1}{2}, \quad \lambda=\pi n+\pi w, \quad|w|=\rho .
$$

Indeed, put

$$
\widetilde{N}(x)=\inf \left\{k: \omega(k)+k^{-(1-1 / b)} \log (e+k) \leqslant \frac{1}{x}\right\} .
$$

Then the right sides in (4.77), (4.78) are smaller than $1 / 2 m$, and (4.74) and (4.73) hold. Therefore, we have proven that in the vertical strips

$$
T_{n}=\{z=\pi n+\pi w:|\operatorname{Re} w| \leqslant 1 / 2\},
$$

the resolvent $R_{\lambda}$ is well-defined outside disks

$$
D_{n}=\left\{z \in \mathbb{C}^{1}:|z-\pi n| \leqslant 1 / 4\right\} .
$$

Of course, by adjusting a constant $C$ in the definition (4.80) we can make the same conclusion by choosing any fixed $r>0$ instead of $1 / 4$, i.e., take disks

$$
D_{n}(r)=\left\{z \in \mathbb{C}^{1}:|z-\pi n| \leqslant r\right\} .
$$

But inequalities (4.77)-(4.78) help us to trap the spectrum $\sigma\left(L_{b c}\right)$ into disks with radiuses $\delta_{n} \rightarrow 0$; compare Section 4.3, (4.29)-(4.32). Indeed, put for example (4.84)

$$
\delta_{n}=\left[\omega(n)+n^{-1-1 / b} \log (1+2 n)\right]^{1 / 3}, \quad N \geqslant n \geqslant N_{1}=4, \quad N_{1}=\widetilde{N}_{1}\left(C_{28} m M\right)
$$

with

$$
\widetilde{N}_{1}(x)=\widetilde{N}\left(x^{3}\right), \quad x \geqslant 1 .
$$

Then (4.80) guarantees, - together with (4.77)-(4.78) that (4.79) holds in the strip $T_{n},|n| \geqslant N_{1}$, outside of the disks

$$
D_{n}\left(\delta_{n}\right)=\left\{z \in \mathbb{C}^{1}:|z-\pi n| \leqslant \delta_{n}\right\} .
$$

9. Therefore, we have proven a more general that Theorem 4.1 statement:

TheOREM 4.5. Let us consider $L_{b c}$ defined by (1.1) and $b c=P e r^{ \pm}$or Dir with a potential $V \in L^{b}, 1<b$ and

$$
V \in H(b, \Omega), \quad V \in K(m, M)
$$

(see (5.10)-(5.13)). Define contour $\Gamma$ by (4.4)-(4.6) where

$$
Y=(C(b) m)^{\frac{1}{b}-1}, \quad X=\pi N+\frac{\pi}{2},
$$

and $N=\widetilde{N}\left(C_{28} m M\right),\left\{\delta_{n}\right\}$ are deined in (4.80) and (4.84). Then

$$
\sigma\left(L_{b c}\right) \subset R(X ; Y) \cup \bigcup_{|n|>N} D\left(n ; \delta_{n}\right) .
$$


10. Remarks in Section 4.6 are general and can be applied under assumptions of Theorem 4.5 as soon as this theorem has been proven. Therefore, we have the same Counting Lemma as Proposition 4.3, i.e.

Proposition 4.6. Under assumptions and notations of Theorem 4.5, the dimensions of Riecz spaces $\operatorname{Im} P_{*}$ and $\operatorname{Im} P_{n},|n|>N$,

$$
\begin{gathered}
P_{*}=\frac{1}{2 \pi i} \int_{\partial R(X, Y)}\left(\lambda-L_{b c}\right)^{-1} d \lambda \\
P_{n}=\frac{1}{2 \pi i} \int_{\partial D_{n}}\left(\lambda-L_{b c}\right)^{-1} d \lambda
\end{gathered}
$$

are given by Table 2 (see Prop. 4.3).

\section{Estimates of Norms of Resolvent and Related Operators in Complex Plane}

0. Proposition 4, inequalities (2.27) and (2.83), were important to make the norm of the square $K^{2}, K=V R_{\lambda}^{0}$, small if $\lambda=\pi n+\pi w,|R e w| \leqslant 1 / 2$, and $n$ large enough. This led us to main results (Counting Lemma) of Section 4 about the contour, or domain, which traps the spectrum $\sigma\left(L_{b c}\right)$. For further results on convergence of spectral decompositions, completeness or uniform boundedness of eigenfunctions (Riesz subspaces), given in the next Section 6, we need more inequalities for norms of resolvents and other operator-valued functions. Of course, we'll repeat basic scheme of Section 2 but now a potential is an $L^{b}$-function, $1<$ $b \leqslant 2$, and operators act from $L^{c}$ into $L^{a}$, with $c$ and $a$ more general than 2 or $\infty$.

1. Notations and general scheme.

If $V \in L^{b}, 1<b \leqslant 2$, as in (2.8)

$$
\left\|V: L^{\infty} \rightarrow L^{b}\right\|=\|v\|_{b} \leqslant m
$$

but an analogue of the norm (2.30), or the space $H(\Omega)$ if $b=2$, is defined on the basis of Hausdorff-Young Theorem ([25], Sect. 12.2, Thm. 2.3):

LEMMA 5.1. (i) If $v \in L^{b}, 1 \leqslant b \leqslant 2$, then its Fourier coefficients sequence

$$
\widetilde{v} \in(\widetilde{v}(k)) \in l^{b^{\prime}}, \quad \frac{1}{b}+\frac{1}{b^{\prime}}=1,
$$

and

$$
\left\|\widetilde{v}\left|l^{b^{\prime}}\|\leqslant C\| v\right| L^{b}\right\|
$$

(ii) If a sequence $f=\left(f_{k}\right) \in l^{d}, 1 \leqslant d \leqslant 2$, then a function

$$
F(x)=\sum f_{k} E_{k}(x) \in L^{d^{\prime}}
$$

and

$$
\left\|F\left|L^{d^{\prime}}\|\leqslant C\| f\right| l^{d}\right\|
$$

These statements hold for standard Fourier series and more generally for any complete orthogonal system $\left\{E_{k}\right\}$ which is uniformly bounded in $L^{\infty}$, i.e.

$$
\left\|E_{k} \mid L^{\infty}\right\| \leqslant a<\infty
$$


A constant $C=C(a)$ in (5.3) and (5.5) depends on $a$ only.

See the proofs and further details in Zygmund, Ch. 12.

Therefore, for $V \in L^{b}, 1<b \leqslant 2$,

$$
\sum|\widetilde{v}(k)|^{b^{\prime}} \leqslant C^{b^{\prime}} m^{b^{\prime}}
$$

and for a given compact $K \subset L^{b}$ these series converges uniformly for $v \in K$. It follows that for some sequence

$$
\Omega_{k} \nearrow \infty, \quad \Omega(0)=1, \quad \Omega(-k)=\Omega(k),
$$

still

$$
M=\sum\left\{\left(\sum(|\widetilde{v}(k)| \Omega(k))^{b^{\prime}}\right)^{1 / b^{\prime}}: v \in K\right\}<\infty .
$$

See details on the choice of $\Omega$ in Lemma 8.2, Sect. 8 below.

With this motivation, as in (1.6)-(1.7) for any sequence (5.8) we define the space

$$
H(b ; \Omega)=\left\{v \in L^{b}:\left\|\widetilde{v} \mid l^{b^{\prime}}(\Omega)\right\|<\infty\right\}
$$

where

$$
\left\|\widetilde{v} \mid l^{b^{\prime}}(\Omega)\right\|=\left(\sum(|\widetilde{v}(k)| \Omega(k))^{b^{\prime}}\right)^{1 / b^{\prime}}, \quad 1 \leqslant b \leqslant 2,
$$

and

$$
\left\|v|H(b ; \Omega)\|=\| v| L^{b}\right\|+\left\|\widetilde{v} \mid l^{b^{\prime}}(\Omega)\right\| .
$$

¿From now on we assume that a potential $V$ is in a bounded set

$$
K(m, M)=\left\{V \in L^{b}\left|\left\|v\left|L^{b}\|\leqslant m,\| v\right| H(b ; \Omega)\right\| \leqslant M\right\} .\right.
$$

For curiosity, let us notice that (5.8) does not guarantee that $K(m, M)$ is a compact set in $L^{b}, 1<b<2$, although this is certainly true if $b=2$.

2. Hölder inequality implies that

$$
\left\|V: L^{a} \rightarrow L^{d}\right\|=\|v\|_{b}
$$

if

$$
\frac{1}{a}+\frac{1}{b}=\frac{1}{d}, \quad \frac{b d}{b-d} \leqslant a \leqslant \infty, \quad 1 \leqslant d \leqslant b .
$$

Of course,

$$
\left\|V \mid L^{\infty} \rightarrow L^{b}\right\|=\|v\|_{b}, \quad 1 \leqslant b \leqslant 2 .
$$

For a multiplier-operator $R=\left(r_{k}\right)$,

$$
R E_{k}=r_{k} E_{k}, \quad k \in \mathbb{Z},
$$

Lemma 5.1, (ii) and (i), implies

Lemma 5.2. If $1 \leqslant a \leqslant 2,2 \leqslant c \leqslant \infty$, then

$$
\left\|R: L^{a} \rightarrow L^{c}\right\| \leqslant C^{2}\left\|r \mid l^{\sigma}\right\|, \quad \frac{1}{\sigma}=\frac{1}{a}-\frac{1}{c} .
$$


Proof. $R$ can be factorized by Lemma 5.1 as

$$
L^{a} \stackrel{J_{(i)}}{\rightarrow} l^{a^{\prime}} \stackrel{r}{\rightarrow} l^{c^{\prime}} \stackrel{J_{(i i)}}{\longrightarrow} L^{c}
$$

with

$$
\left\|r: l^{a^{\prime}} \rightarrow L^{c^{\prime}}\right\|=\left\|r \mid l^{\sigma}\right\|
$$

(compare (5.14)) where $J$ are Young operators from Lemma 5.1. It implies (5.18) with $C^{2}$ coming from (5.3).

If $R=R_{\lambda}^{0}$ is a resolvent of $L^{0}$ then Lemma 5.2 tells us that

$$
\left\|R_{\lambda}^{0}: L^{a} \rightarrow L^{c}\right\| \leqslant C^{2}\left\|r_{\lambda}^{0} \mid l^{\sigma}\right\| \leqslant C^{2} A(\sigma ; \mu), \quad 1 \leqslant a \leqslant 2 \leqslant c \leqslant \infty,
$$

where $1 \geqslant 1 / \sigma=1 / a-1 / c \geqslant 0$.

Of course, in the case $a=1, c=\infty$ an operator $R_{\lambda}^{0}$ is unbounded. The inequality (5.21) holds with $A(1 ; \mu)=\infty$ but it is meaningless.

(3.1), (5.14) and (5.21) lead us to

Lemma 5.3. For $1 \leqslant a \leqslant 2,1 \leqslant d \leqslant b \leqslant 2$

$$
\left\|V R_{\lambda}^{0}: L^{a} \rightarrow L^{d}\right\| \leqslant C^{2}\left\|r_{\lambda}^{0} \mid l^{\sigma}\right\| m \leqslant C^{2} A(\sigma ; \mu) m,
$$

where

$$
\frac{1}{\sigma}=\frac{1}{a}-\frac{1}{d}+\frac{1}{b}
$$

Proof. As in (5.15) we factorize $V R_{\lambda}^{0}$ in the following sequence with $c^{\prime}, 1 \leqslant$ $c^{\prime} \leqslant 2$

$$
L^{a} \stackrel{J_{(i)}}{\rightarrow} l^{a^{\prime}} \stackrel{r}{\rightarrow} l^{c^{\prime}} \stackrel{J_{(i i)}}{\rightarrow} L^{c} \stackrel{V}{\rightarrow} L^{d} .
$$

By (5.14)-(5.15) ( $a$ there should be changed to $c$ ) we can choose $c$ by

$$
\frac{1}{c}+\frac{1}{b}=\frac{1}{d}
$$

so $\frac{1}{c} \leqslant \frac{1}{d} \leqslant \frac{1}{2}, \infty \geqslant c \geqslant 2$ and $1 \leqslant c^{\prime} \leqslant 2$ permits us to apply Lemma 5.1 (ii).

Notice that $1 \geqslant 1 / d \geqslant 1 / b \geqslant 1 / 2$ so

$$
\frac{1}{d}-\frac{1}{b} \leqslant 1-\frac{1}{2} \leqslant \frac{1}{2} \text { and } 0 \leqslant \frac{1}{\sigma}=\frac{1}{a}-\left(\frac{1}{d}-\frac{1}{b}\right)<1 .
$$

By Lemma 5.2 factorization (5.24) leads to (5.22).

3. Now we'll estimate the norm

$$
\left\|R^{0} V R^{0}: L^{b} \rightarrow L^{c}\right\|, \quad 1<b \leqslant 2 \leqslant c \leqslant \infty .
$$

We could consider the index $a \neq b$ but with complete understanding of our method it can be easily adjusted. The choice of $b$ (with $V \in L^{b}$ and $V \in K(m, M)$ from (5.13)) is important for further applications.

Of course, keeping in mind inequalities (5.14) and (5.21) we can factorize $R^{0} V R^{0}$ as

$$
L^{b} \stackrel{R_{\lambda}^{0}}{\rightarrow} L^{\infty} \stackrel{L}{\rightarrow} L^{b} \stackrel{R_{\lambda}^{0}}{\rightarrow} L^{c}
$$

and it leads to estimates $(1 / \tau=1 / b-1 / c)$

(5.29)

$\left\|R_{\lambda}^{0} V R \lambda^{0}: L^{b}\right\| \leqslant\left\|R_{\lambda}^{0}: L^{b} \rightarrow L^{\infty}\right\| \times m \times\left\|R_{\lambda}^{0}: L^{b} \rightarrow L^{c}\right\| \leqslant C^{4} m A(b ; \mu) A(\tau ; \mu)$. 
But functions $A$ are periodic, $A(\mu)=A(\mu+1)$, and (5.29) does not give any decay along real axis when $n \rightarrow \infty(\mu=n+w,|\operatorname{Re} w| \leqslant 1 / 2,|\mu|=\rho)$. Still, by Lemma 3.1, inequality (3.21), we get from (5.29)

$$
\begin{aligned}
\left\|R_{\lambda}^{0} V R_{\lambda}^{0}: L^{b} \rightarrow L^{c}\right\| & \leqslant C^{4} \cdot C_{3}(b) C_{3}(\tau) m \cdot\left(\frac{1}{\rho}+\frac{1}{\rho^{1-1 / b}}\right)\left(\frac{1}{\rho}+\frac{1}{\rho^{1-1 / \tau}}\right) \leqslant \\
& \leqslant C(b ; c) m\left(\frac{1}{\rho^{2}}+\frac{1}{\rho^{2(1-1 / b)+1 / c}}\right) .
\end{aligned}
$$

Now we'll give an analogue of Proposition 2.4, Section 2, in the case of $L^{b}$-potential.

Proposition 5.4. Let a potential $V$ in (1.1) be from the set $K(m, M) \in(5.13)$. Then $(1<b \leqslant 2)$ with $0 \leqslant 1 / \tau=1 / a-1 / c<1$

$$
w^{*}=\left\|R_{\lambda}^{0} V R_{\lambda}^{0}: L^{a} \rightarrow L^{c}\right\| \leqslant K_{1}(a, b, c) A(\tau, \mu) A_{1}(b, \mu)
$$

if

(a) $1 \leqslant a \leqslant 2 \leqslant c \leqslant \infty$ but $\{a, c\} \neq\{1, \infty\}$ with

$$
0<K_{1}(a, b, c)<\infty
$$

and

(b) $b c=\operatorname{Per}^{ \pm}$or Dir $\mathcal{H}^{-}$.

In the case Dir $\mathcal{H}^{-}$or general Dir the estimate (5.33) holds

$$
\left\|R_{\lambda}^{0} V R_{\lambda}^{0}: L^{a} \rightarrow L^{c}\right\| \leqslant K_{2}(a, b, c)\left[A(\tau, \mu)+A(a, \mu) A\left(c^{\prime}, \mu\right)\right] A_{1}(b, \mu)
$$

with $0<K_{2}<\infty$ if

(c) $1<a \leqslant 2 \leqslant c<\infty$.

Finally, if $c=\infty, 1<a \leqslant 2,1<b \leqslant 2$,

$$
\left\|R_{\lambda}^{0} V R_{\lambda}^{0}: L^{a} \rightarrow L^{\infty}\right\| \leqslant K_{3}(a, b) A(a, \mu) A_{2}(b, \mu) .
$$

(Functions $A, A_{1}$ and $A_{2}$ are defined in Section 3.)

Proof. As in Section 2, - see the proof of Proposition 2.4, - we give an algebraic representation of this operator $W=R_{\lambda}^{0} V R_{\lambda}^{0}$ and factorize it in the following way

$$
L^{a} \stackrel{J_{1}}{\rightarrow} l^{a^{\prime}} \stackrel{W}{\rightarrow} l^{c^{\prime}} \stackrel{J_{2}}{\rightarrow} L^{c}
$$

so by (5.3), (5.5)

$$
\left\|W: L^{a} \rightarrow L^{c}\right\| \leqslant C^{2}\left\|w: l^{a^{\prime}} \rightarrow L^{c^{\prime}}\right\| .
$$

But $W=W_{\lambda}(V)$ depends in a linear way on $V \in H(b, \Omega)$ and it is not just a multiplier like $R$ in lemma 5.2.

But we know (see (2.44) and (2.61)) how this operator acts on eigenvectors of $L_{b c}^{0}$. There are two cases, and we'll consider them separately.

Case $1\left(\right.$ Per $^{ \pm}$or Dir $\left.\mathcal{H}^{+}\right)$. By (2.44) or (5.81)

$$
W e_{j}=\alpha \sum_{k} \frac{1}{\mu-j} g_{k} \omega_{k} \frac{1}{\mu-(k-j)} e_{k-j}
$$

where

$$
V=\sum g_{k} \omega_{k} E_{k} \in K(m, M), \quad \omega_{k}=\frac{1}{\Omega_{k}}
$$


SO

$$
\left(\sum\left|g_{k}\right|^{b^{\prime}}\right)^{1 / b^{\prime}} \leqslant M
$$

By (5.36) and (5.38) and duality for $l^{c^{\prime}}$, the norm $\left\|W: L^{a} \rightarrow L^{c}\right\|$

$$
\left\|W: L^{a} \rightarrow L^{c}\right\| \leqslant C^{2} M \alpha w^{*}
$$

does not exceed $C^{2} M \omega^{*} \alpha$ where

$$
\begin{gathered}
w^{*}=\sup \left\{W(f, g, t)\left|\left\|f\left|l^{a^{\prime}}\|\leqslant 1,\| g\right| l^{b^{\prime}}\right\| \leqslant 1,\left\|t \mid l^{c}\right\| \leqslant 1\right\},\right. \\
W(f, g, t)=\sum_{j, k} f_{j} g_{k} \omega_{k} \frac{1}{\mu-j} \cdot \frac{1}{\mu-(k-j)} t_{k-j},
\end{gathered}
$$

and sequences $f, g, t$ are finite so there is no problems with (convergence of) infinite series. Let us majorize this ternary form (5.41). As in (2.50)

$$
\frac{1}{\mu-j} \cdot \frac{1}{\mu-(k-j)}=\frac{1}{2 \mu-k}\left[\frac{1}{\mu-j}+\frac{1}{\mu-(k-j)}\right]
$$

and

$$
W(f, g, t)=\sum_{k} g_{k} \frac{\omega_{k}}{2 \mu-k} x_{k}
$$

where

$$
x_{k}=\sum_{j} f_{j} t_{k-j}\left(\frac{1}{\mu-j}+\frac{1}{\mu-(k-j)}\right) .
$$

For any $k\left\|t_{k-.}\left|l^{c}\|=\| t\right| l^{c}\right\| \leqslant 1$, and sequences $\left\{\frac{1}{\mu-.}\right\}$ and $\left\{\frac{1}{\mu-(k-\cdot)}\right\}$ have the same norms in $l^{p}$-spaces, in particular in $l^{\tau}$,

$$
\frac{1}{\tau}=\frac{1}{a}-\frac{1}{c} .
$$

Notice that with $1<a \leqslant 2 \leqslant c \leqslant \infty$ we have

$$
1>\frac{1}{a} \geqslant \frac{1}{2} \geqslant \frac{1}{c} \geqslant 0
$$

so $0 \leqslant 1 / \tau \leqslant 1 / a<1$ and $\tau \neq 1$.

Hölder inequality for the factors in (5.44) guarantees that for any $k \in \mathbb{Z}, m \notin \mathbb{Z}$

$$
\left|x_{k}\right| \leqslant\left\|f\left|l^{a^{\prime}}\|\cdot\| t\right| l^{c}\right\| \cdot 2 A(\tau, \mu) \leqslant 2 A(\tau, \mu)
$$

because

$$
\frac{1}{a^{\prime}}+\frac{1}{c}+\frac{1}{\tau}=1
$$

by (5.45).

Now, by (5.43)

$$
\begin{gathered}
|W(f, g, t)| \leqslant 2 A(\tau, \mu) \sum_{k} \frac{\omega_{k}}{|2 \mu-k|} \cdot\left|g_{k}\right| \leqslant 2 A(\tau, \mu)\left\|g \mid l^{b^{\prime}}\right\| A_{1}(b ; \mu) \leqslant \\
\leqslant 2 A(\tau, \mu) A_{1}(b, \mu) .
\end{gathered}
$$


Like in (5.30) we use Lemmas 3.1 and 3.3, or inequalities (5.21) and (5.25) to write (5.50) in terms $n$ and $\rho$ more explicitly:

$$
|W(f, g, t)| \leqslant K_{18} C_{3}(\tau)\left(\frac{1}{\rho}+\frac{1}{\rho^{1-1 / \tau}}\right) \cdot\left(\frac{\omega(2 n)}{\rho}+C_{4}(b) \frac{\omega(n)}{1+\rho^{1+\rho^{1-1 / b}}}+\frac{3 n^{1 / b}}{n+\rho}\right)
$$

or if $\rho \geqslant \frac{1}{4}$

$$
\left\|R_{\lambda}^{0} V R_{\lambda}^{0}: L^{a} \rightarrow L^{c}\right\| \leqslant K(a, b, c) \rho^{(1 / a-1 / c)^{-1}}\left[\omega(n) \rho^{-(1-1 / b)}+\frac{n^{1 / b}}{n+\rho}\right] .
$$

Case $2\left(\right.$ Dir $\left.\mathcal{H}^{-}\right)$. By $(2.60)$ or $(2.61)$

$$
W u_{j}=\beta \sum_{k l} \omega_{k} g_{k} \frac{1}{k-j-l} \cdot \frac{1}{\mu-j} \cdot \frac{1}{\mu-l} u_{l}
$$

where

$$
V=\sum g_{k} \omega_{k} U_{k}
$$

(see (2.37), (2.59), (2.62)) and $V \in K(m, M)$. As in (5.38)-(5.41) (5.39) holds but instead of (5.41) we need analyze a ternary form

$$
W(f, g, t)=\sum_{j k l} f_{j} g_{k} t_{l} \omega_{k} \frac{1}{\mu-j} \cdot \frac{1}{\mu-l} \cdot \frac{1}{k-j-l} .
$$

As we do often $(j+l-k$ are odd $)$, write

$$
\begin{aligned}
& \frac{1}{\mu-j} \cdot \frac{1}{\mu-l} \cdot \frac{1}{k-j-l}=\frac{1}{2 \mu-k}\left[\left(\frac{1}{\mu-l}+\frac{1}{\mu-(k-l)}\right)\left(\frac{1}{k-j-l}-\frac{1}{\mu-j}\right)\right] \\
& \text { So } \\
& w=W(f, g, t)=\sum_{k} g_{k} \frac{\omega_{k}}{2 \mu-k} x_{k},
\end{aligned}
$$

where

$$
x_{k}=x_{k}^{1}+x_{k}^{2}+x_{k}^{3}+x_{k}^{4}
$$

and $x_{k}^{\gamma}, \gamma=1,2,3,4$ are written explicitly below in (5.59), (5.64), (5.66).

Terms $x_{k}^{1}$.

$$
x_{k}^{1}=\sum_{j l} f_{j} t_{l} \frac{1}{\mu-l} \cdot \frac{1}{k-j-l}=\sum_{l} \frac{t_{l}}{(\mu-l} \sum_{j} \frac{f_{j}}{k-l-j}=\sum \frac{t_{l}}{\mu-l} \cdot \widetilde{f}(k-l)
$$

where $\tilde{f}$ is a Hilbert transform of $f$.

With $1<a \leqslant 2$ and $2 \leqslant a^{\prime}<\infty$

$$
\left\|\tilde{f}\left|l^{a^{\prime}}\|\leqslant h(a)\| f\right| l^{a^{\prime}}\right\| \quad \text { and } \quad\left\|\widetilde{f}(k-\cdot)\left|l^{a^{\prime}}\|=\| \widetilde{f}\right| l^{a^{\prime}}\right\|, \quad \forall k .
$$

Therefore, by Hölder inequality

$$
\left|x_{k}^{1}\right| \leqslant\left\|t\left|l^{c}\|\cdot\| \widetilde{f}\right| l^{a^{\prime}}\right\| \cdot A(\tau, \mu)
$$

with

$$
\frac{1}{c}+\frac{1}{a^{\prime}}+\frac{1}{\tau}=1, \quad \text { i.e. } \quad \frac{1}{\tau}=\frac{1}{a}-\frac{1}{c} \leqslant \frac{1}{a}<1,
$$

and

$$
\left|x_{k}^{1}\right| \leqslant h(a) A(\tau, \mu)
$$


Terms $x_{k}^{2}$.

$$
x_{k}^{2}=-\sum f_{k} t_{l} \frac{1}{\mu-l} \cdot \frac{1}{\mu-j}=-\left(\sum \frac{f_{j}}{\mu-j}\right)\left(\sum \frac{t_{l}}{\mu-l}\right)
$$

and

$$
\left|x_{k}^{2}\right| \leqslant A(a, \mu) \cdot A\left(c^{\prime}, \mu\right)
$$

with $1<a \leqslant 2$ and $1 \leqslant c^{\prime} \leqslant \infty$.

This inequality is good if $1<c^{\prime}$, i.e. $c<\infty$, but the case $c=\infty$ will require a special statement.

Terms $x_{k}^{3}$ are estimated in the same way as $x_{k}^{1}$.

$$
\begin{gathered}
x_{k}^{3}=\sum_{j k} f_{j} t_{l} \frac{1}{\mu-(k-l)} \cdot \frac{1}{k-j-l}= \\
=\sum_{l} \frac{t_{l}}{\mu-(k-l)} \sum_{j} \frac{f_{j}}{(k-l)-j}=\sum_{l} t_{l} \cdot \frac{1}{\mu-(k-l)} \cdot \widetilde{f}(k-l),
\end{gathered}
$$

and as in (5.60)-(5.61) for $x_{k}^{1}$

$$
\left|x_{k}^{3}\right| \leqslant\left\|t\left|l^{c}\|\cdot h(a)\| f\right| l^{a^{\prime}}\right\| A(\tau, \mu) \leqslant h(a) A(\tau, \mu),
$$

with $\tau$ from (5.62).

Terms $x_{k}^{4}$ are estimated as $x_{k}^{2}$

$$
x_{k}^{4}=-\sum f_{j} t_{l} \frac{1}{\mu-(k-l)} \cdot \frac{1}{\mu-j}=-\left(\sum \frac{f_{j}}{\mu-j}\right)\left(\sum \frac{t_{l}}{\mu-(k-l)}\right)
$$

and

$$
\left|x_{k}^{4}\right| \leqslant A(a, \mu) A\left(c^{\prime}, \mu\right) .
$$

If we collect (5.63), (5.65), (5.67) and (5.69), with $\tau \in(5.62)$, we come to the inequality

$$
\left|x_{k}\right| \leqslant C_{15}\left[A(a, \mu) \cdot A\left(c^{\prime}, \mu\right)+A(\tau, \mu)\right]<\infty
$$

if $c \neq \infty$.

Now, by (5.57) - like in (5.43) and (5.49)

$$
|w| \leqslant X \cdot\left\|g \mid l^{b^{\prime}}\right\| \cdot A(b, \mu) .
$$

It leads to inequalities (compare (5.50)-(5.52))

$$
\begin{aligned}
|w| \leqslant C_{23}[ & \left.\left(\frac{1}{\rho}+\frac{1}{\rho^{1-1 / a}}\right)\left(\frac{1}{\rho}+\frac{1}{\rho^{1 / c}}\right)+\left(\frac{1}{\rho}+\frac{1}{\rho^{1-1 / \tau}}\right)\right] \times \\
& \times\left(\frac{\omega(n)}{\rho}+\frac{\omega(n)}{\rho^{1-1 / b}}+\frac{n^{1 / b}}{n+\rho}\right),
\end{aligned}
$$

and if $\rho \geqslant 1 / 4$

$$
\left\|R_{\lambda}^{0} V R_{\lambda}^{0}: L^{a} \rightarrow L^{c}\right\| \leqslant C_{24} \rho^{-1+(1 / a-1 / c)}\left[\omega(n) \rho^{-(1-1 / b)}+\frac{n^{1 / b}}{n+\rho}\right] .
$$

It looks as (5.52) but the difference comes if $c=\infty$. In (5.52) a constant $K(a, b, c)$ is finite if

$$
1<a \leqslant 2 \leqslant c \leqslant \infty, \quad 1<b \leqslant 2 \quad \text { or } \quad 1 \leqslant a \leqslant 2 \leqslant c<\infty
$$

(see (5.46)), but in (5.65) and (5.69) $c=\infty$ is not acceptable so $C_{24}=\infty$ if $c=\infty$. 
But the case $c=\infty$ is important for us and we now consider (5.55) for $c=\infty$ so

$$
\left|t_{l}\right| \leqslant 1
$$

Now instead of (5.57) we use a representation

$$
w=\sum_{k} g_{k} \frac{\omega_{k}}{2 \mu-k} \cdot x_{k}
$$

where

$$
x_{k}=\sum_{l} t_{l}\left(\frac{1}{\mu-l}+\frac{1}{\mu-(k-l)}\right) \sum_{j} f_{j}\left(\frac{1}{k-l-j}-\frac{1}{\mu-j}\right) .
$$

Notice as in (5.59)-(5.60) that

$$
\sum_{j} f_{j} \frac{f_{j}}{k-l-j}=\widetilde{f}(k-l),
$$

and

$$
\sum_{j} f_{j} \frac{1}{\mu-j}=\widetilde{f}(\mu)
$$

$\mathrm{SO}$

$$
|\widetilde{f}(\mu)| \leqslant\left\|f \mid l^{a^{\prime}}\right\| A(a, \mu) .
$$

But the sequence (5.78) for each $k$ can be considered as a shift

$$
\{\tilde{f}(k-\cdot)\} \in l^{a^{\prime}}, \quad 1 \leqslant a^{\prime}<\infty,
$$

of a Hilbert transform of $f$, and

$$
\left\|\widetilde{f}(k-\cdot) \mid l^{a^{\prime}}\right\| \leqslant h(a), \quad \forall k .
$$

Therefore, with (5.75)

$$
\begin{gathered}
\text { (5.83) } \quad\left|x_{k}\right| \leqslant\left|\sum_{l} t_{l}\left(\frac{1}{\mu-l}+\frac{1}{\mu-(k-l)}\right)(\widetilde{f}(k-l)+\widetilde{f}(\mu))\right| \leqslant \\
\leqslant \sum_{l}|\widetilde{f}(k-l)|\left(\frac{1}{|\mu-k|}+\frac{1}{|\mu-(k-l)|}\right)+|\widetilde{f}(\mu)| \sum_{l}\left|\frac{1}{\mu-l}+\frac{1}{\mu-(k-l)}\right| \leqslant X, \\
(5.84) \quad X=2 h(a) A(a, \mu)+A(a, \mu) B_{*}(2 n-k ; \omega) .
\end{gathered}
$$

The last factor $B_{*}$ comes from the sum

$$
\begin{gathered}
\sum_{l}\left|\frac{1}{n+w-l}+\frac{1}{n+w-k+l}\right|= \\
=\sum\left|\frac{1}{w+j}+\frac{1}{(2 n-k)-j+w}\right|=B_{*}(2 n-k ; w) .
\end{gathered}
$$

We use (5.83)-(5.84) to estimate $w$ in (5.76); we have

$|w| \leqslant C A(a, \mu) \sum_{k}\left|g_{k}\right| \frac{\omega_{k}}{|2 n-k+2 \omega|} B_{*}(2 n-k ; \omega) \leqslant A(a, \mu) A_{2}(b, \mu), \quad 1<b \leqslant 2$.

It completes the proof of Proposition 5.4. 
Inequalities (5.51), (5.52) simplified (5.31) by writing the right side of this inequality as a function of $\rho=|\mu|$. Let us do the same for (5.33) and (5.34).

Corollary 5.5. If (5.32) holds and $b c=\operatorname{Per}^{ \pm}$or Dir $\mathcal{H}^{+}$then for $\rho \geqslant 1 / 4$

$$
\omega^{*} \leqslant K(a, b, c) \rho^{-2+(1 / a+1 / b)-1 / c}\left[\omega(n)+\left(\frac{n}{\rho}\right)^{1 / b} \cdot \frac{1}{1+n / \rho}\right] .
$$

If

$$
1<a \leqslant 2 \leqslant c<\infty
$$

and $b c=$ Dir then the same inequality (5.87) holds with $K_{7}(a, b, c)$ finite in (5.88).

Finally, if $c=\infty$, for $\rho \geqslant 1 / 4$

$$
\omega^{*} \leqslant K_{8} \rho^{-(1-1 / a)}\left[\omega(n) \frac{1+\log (1+\rho)}{1+\rho^{1-1 / b}}+\frac{n^{1 / b} \log (1+2 n)}{n+\rho}\right] .
$$

If $\rho$ is small such estimates are important for us (see Sections 4.3, 4.4, 4.5 inequalities (4.29)-(4.32) or (4.40), (4.41)-(4.43)) if we want to localize the spectrum $\sigma\left(L_{b c}\right)$. It has been used and explained in Sections 4.7-4.9.

Corollary 5.6. For small $\rho$

$$
w^{*} \leq K_{g} \frac{1}{\rho}\left[\frac{\omega(2 n)}{\rho}+\omega(n)+n^{\frac{1}{b}-1}\right] .
$$

Now we are ready to analyze problems mentioned in Section 5.0. It is done in the next Sections 6 and 7.

\section{Spectral Expansions and Their Convergence}

0. Proposition 2.4, inequalities (2.27) and (2.83) in Section 2, have been important in making the norm of the square $K^{2}, K=V R_{\lambda}^{0}$, small if $n$ large enough. This led us to main results of the previous section (Theorem 4.1, 4.2 and Proposition 4.3).

Now we will use Proposition 5.4 to get $L^{2}$ - and $L^{\infty}$-estimates of deviations $P f-P^{0} f$ where $P, P^{0}$ are Riesz projections (6.4). It will allow us to prove a series of statements on convergence of spectral expansions

$$
f=P_{*} f+\sum_{|n|>N} P_{n} f
$$

where (see (4.56) and (4.57), Section 4 or (1.9), (1.10), Section 1)

$$
P_{*}=P_{*}(1), \quad P_{n}=P_{n}(1) .
$$

At the same time we use notations

$$
P_{*}^{0}=P_{*}(0), \quad P_{n}^{0}=P_{n}(0)
$$

for projections in the case of zero potential $V$ (see Section 1.2).

1. Projections for Riescz subspaces are defined by Cauchy integral

$$
P=\frac{1}{2 \pi i} \int_{C} R_{\lambda} d \lambda
$$

where $C$ is a properly chosen contour,

$$
C \cap \sigma\left(L_{b c}\right)=\varnothing \quad \text { and } \quad C \cap \sigma\left(\begin{array}{l}
0 \\
b c
\end{array}\right)=\varnothing .
$$


Therefore

$$
P-P^{0}=\frac{1}{2 \pi i} \int_{C}\left(R_{\lambda}-R_{\lambda}^{0}\right) d \lambda .
$$

As through this paper we follow the perturbation scheme so

$$
\begin{gathered}
R_{\lambda}-R_{\lambda}^{0}=R_{\lambda}\left(I-V R_{\lambda}^{0}\right)^{-1}-R_{\lambda}^{0}= \\
=R_{\lambda}^{0} V R_{\lambda}^{0}\left(I-V R_{\lambda}^{0}\right)^{-1}=R_{\lambda}^{0} V R_{\lambda}^{0}\left(I+V R_{\lambda}^{0}\right)\left(I-V R_{\lambda}^{0} V R_{\lambda}^{0}\right)^{-1} .
\end{gathered}
$$

Put

$$
\begin{gathered}
a(\lambda)=\left\|V R_{\lambda}^{0}\right\| . \\
f(\lambda)=\left\|R_{\lambda}^{0} V R_{\lambda}^{0}: L^{2} \rightarrow L^{\infty}\right\|,
\end{gathered}
$$

and

$$
f_{0}(\lambda)=\left\|R_{\lambda}^{0} V R_{\lambda}^{0}: L^{2} \rightarrow L^{2}\right\|
$$

(By Lemma 5.2

$$
f(\lambda) \leqslant a(\lambda) A(\mu)
$$

and

$$
f_{0}(\lambda) \leqslant a(\lambda) A(\infty, \mu)
$$

Then if (compare (4.9))

$$
a(\lambda) \leqslant \frac{1}{2}, \quad \lambda \in \gamma
$$

we have

$$
\left\|\frac{1}{2 \pi i} \int_{\gamma}\left(R_{\lambda}-R_{\lambda}^{0}\right) d \lambda\right\|_{2 \rightarrow \infty} \leqslant \frac{2}{2 \pi} \int_{\gamma} f(\lambda)|d \lambda|
$$

and the norm of the same operator in $L^{2}$

$$
\left\|\frac{1}{2 \pi i} \int_{\gamma}\left(R_{\lambda}-R_{\lambda}^{0}\right) d \lambda\right\|_{2 \rightarrow 2} \leqslant \frac{1}{\pi} \int f_{0}(\lambda) d \lambda .
$$

If we need to use (compare (4.10)) an assumption

$$
\left\|K^{2}\right\|=\left\|V R_{\lambda}^{0} V R_{\lambda}^{0}\right\| \leqslant \frac{1}{2}, \quad \lambda \in \gamma,
$$

or just this fact when it holds, then a second representation in (6.7) implies

$$
\left\|\frac{1}{2 \pi i} \int_{\gamma}\left(R_{\lambda}-R_{\lambda}^{0}\right) d \lambda\right\|_{2 \rightarrow \infty} \leqslant \frac{2}{2 \pi} \int_{\gamma}(1+a(\lambda)) f(\lambda)|d \lambda|
$$

and the norm of the same operator in $L^{2}$ is majorized in the following way

$$
\left\|P_{\gamma}-P_{\gamma}^{0}\right\|_{2 \rightarrow 2} \leqslant \frac{1}{\pi} \int_{\gamma}(1+a(\lambda)) f_{0}(\lambda)|d \lambda| .
$$


By $(6.6)$

$$
\begin{gathered}
\left\|P-P^{0}\right\|_{2 \rightarrow \infty}=\frac{1}{2 \pi} \int_{C}\left\|R_{\lambda}-R_{\lambda}^{0}\right\|_{2 \rightarrow \infty} d|\lambda|, \\
\left\|P-P^{0}\right\|_{2 \rightarrow 2} \leqslant \frac{1}{2 \pi} \int_{C}\left\|R_{\lambda}-R_{\lambda}^{0}\right\|_{2 \rightarrow 2}|d \lambda|,
\end{gathered}
$$

and if we succeed to split $C$ into pieces $\gamma$ where (6.13) or (6.16) holds we could use (6.14) and (6.17) to get a good estimate of the right side in (6.19) or (6.20). This is our approach to finding estimates of the norms $\left\|P-P^{0}\right\|_{2 \rightarrow 2}$ or $\left\|P-P^{0}\right\|_{2 \rightarrow \infty}$ for different contours $C$ in (6.6).

2. With notations (6.1)-(6.3) let us define partial sums $(k, n>N)$

$$
S_{k n} F=P_{*} F+\sum_{\substack{-k \leqslant j \leqslant n \\|j|>N}} P_{j} F
$$

and

$$
S_{k n}^{0} F=P_{*} F+\sum_{\substack{k \leqslant j \leqslant n \\|j|>N}} P_{j}^{0} F
$$

of spectral decompositions for $L_{b c}$ and $L_{b c}^{0}$.

THEOREM 6.1. Under the assumptions and notations of Theorems 4.1 and 4.2 there exist a sequence $\left\{\varepsilon_{n}>0\right\}, \varepsilon_{n} \searrow 0(n \rightarrow \infty)$ such that

$$
\left\|\left(S_{k n}-S_{k n}^{0}\right) F\right\|_{2} \leqslant(\varepsilon(k)+\varepsilon(n))\|F\|_{2} .
$$

The series (6.21) converges in $L^{2}$, i.e.

$$
\lim _{\substack{k \rightarrow \infty \\ n \rightarrow \infty}} S_{k n} F=F, \quad \forall F \in \mathcal{H} .
$$

Proof. We've used to consider $b c=P e r^{ \pm}$and $\operatorname{Dir} \mathcal{H}^{+}$or $b c=\operatorname{Dir} \mathcal{H}^{-}$and general Dir differently but now the argument is the same. Only more complicated inequalities (2.83) and (2.94) than (2.27) or (2.53) give a little bit different sequences $\left\{\varepsilon_{n}\right\}$ for $(6.23)$.

By Theorems 4.1 and 4.2 we can choose

$$
\begin{gathered}
C=\Gamma_{k n}=\partial \Pi \cup \bigcup_{-k \leqslant j \leqslant n} C_{j} \\
|j|>N
\end{gathered}
$$

in (6.6) to present

$$
S_{k n}-S_{k n}^{0}=\frac{1}{2 \pi i} \int_{\Gamma k n}\left(R_{\lambda}-R_{\lambda}^{0}\right) d \lambda, \quad n \geqslant k \geqslant N .
$$

The contour $\Gamma_{k n}$ lies in the vertical strip

$$
L_{k n}=\left\{z \in \mathbb{C}^{1}:-\left(k+\frac{1}{2}\right) \pi \leqslant \operatorname{Re} z \leqslant\left(n+\frac{1}{2}\right) \pi\right\}
$$


and both resolvents $R_{\lambda}$ and $R_{\lambda}^{0}$ are analytic there (by Theorems 4.1 and 4.2) outside of the set

$$
D^{k n}=\Pi \cup \bigcup_{j \in J(k, n)} D\left(\pi j ; \delta_{j}\right)
$$

where

$$
J(k, n)=\{j:|j|>N,-k \leqslant j \leqslant n\} .
$$

Therefore, we can transform - without leaving $L_{k n} \backslash D^{k n}$ - this contour to $\Delta$ preserving the representation (6.26) but choosing $\Delta$ in such a way that it will enable us to give good estimates of the type (6.15) or (6.20) on different pieces of $\Delta$.

3. To realize this approach let us choose (wlog we assume $k \leqslant n$ )

$$
\Delta=\partial \Pi_{k n}(H)
$$

where

$$
\Pi_{k n}(H)=\left\{z \in \mathbb{C}^{1}:-\left(k+\frac{1}{2}\right) \pi \leqslant \operatorname{Re} z \leqslant\left(n+\frac{1}{2}\right) \pi,|\operatorname{Im} z| \leqslant H\right\}
$$

so $\Delta$ is a union of six intervals

$$
\begin{gathered}
\gamma^{ \pm}=\left\{z \in \Pi_{k n}(H): \operatorname{Im} z= \pm H\right\}, \\
\gamma_{j}^{ \pm}=\left\{z \in \mathbb{C}^{1}: \operatorname{Re} z=\left(j+\frac{1}{2}\right) \pi, 0 \leqslant \pm \operatorname{Im} z \leqslant H\right\}, \quad j=-k \text { or } n .
\end{gathered}
$$

If $H$ is large, certainly we want

$$
H>4(1+50 m)^{2}, \quad m=\|V\|_{2},
$$

Proposition 2.3 of Section 2 guarantees that $\gamma^{ \pm}$lie outside of the horizontal strip (2.19), i.e. $|\operatorname{Im} z| \geqslant Y$ and by (2.19)

$$
a(\lambda) \leqslant \frac{4 m}{H^{1 / 2}}<\frac{1}{2}
$$

and by (6.12)

$$
f_{0}(\lambda) \leqslant 4 m H^{-3 / 2}
$$

Therefore, by (6.13) and (6.15)

$$
\left\|\frac{1}{2 \pi i} \int_{\gamma^{ \pm}}\left(R_{\lambda}-R_{\lambda}^{0}\right) d \lambda\right\| \leqslant \pi(1+n+k) \cdot \frac{1}{\pi} \cdot 4 m H^{-3 / 2} .
$$

On $\gamma_{j}^{ \pm}$we are under inequalities (6.16) and (6.18) - don't forget that $|i|>N$ — so by (6.18) and (6.35)

$$
\left\|\frac{1}{2 \pi i} \int_{\gamma_{j}^{ \pm}}\left(R_{\lambda}-R_{\lambda}^{0}\right) d \lambda\right\| \leqslant \frac{1}{\pi} \int_{0}^{H} 2 f_{o}\left(x_{j}+i t\right) d t
$$

and by (6.12) and (2.11)

$$
f_{0}(\lambda) \leqslant 2 A(\infty, \mu) A(2, \mu) \cdot m \quad \text { if } b c=\operatorname{Per}^{ \pm} \text {or } \operatorname{Dir}^{-}
$$


or by $(2.95)$ for $b c=D i r$

$$
f_{0}(\lambda) \leqslant\left[10 A(\infty, \mu) A(\mu)+2 A^{2}(\mu) A_{1}(n, w)\right] \cdot M .
$$

The right side in (6.40) is larger than the right side in (6.39) so we'll use in any case the inequality (6.40). It guarantees that $\left(x_{j}=\left(k+\frac{1}{2}\right) \pi\right)$

$$
f_{0}\left(x_{j}+i t\right) \leqslant \widetilde{C}\left\{\frac{\omega(j)}{(1+t)^{3 / 2}}+\frac{1}{j+t}\left(\frac{j^{1 / 2}}{1+t}+\frac{1}{(1+t)^{1 / 2}}\right)\right\} .
$$

Let us deal with each of three integrals we'll get when we substitute (6.41) into the right side of (6.38). For any $H>0$

$$
\int_{0}^{H} \frac{\omega(j)}{(1+t)^{3 / 2}} d t<\omega(j) \int_{0}^{\infty} \frac{d t}{(1+t)^{3 / 2}}=2 \omega(j) .
$$

Next, for any $H>0$

$$
\int_{0}^{H} \frac{j^{1 / 2} d t}{(j+t)(1+t)}=\int_{0}^{H / j} \frac{j^{1 / 2} \cdot j d \xi}{j(1+\xi) j\left(\frac{1}{j}+\xi\right)}<
$$

$$
j^{-1 / 2} \int_{0}^{\infty} \frac{d \xi}{(1+\xi)\left(\frac{1}{j}+\xi\right)}<j^{-1 / 2}\left(\int_{1}^{\infty} \frac{d \xi}{\xi^{2}}+\int_{0}^{1} \frac{d \xi}{\frac{1}{j}+\xi}\right)=j^{-1 / 2}(1+\log (1+j)) .
$$

Finally, for any $H>0$

$$
\begin{gathered}
\int_{0}^{H} \frac{d t}{(j+t)(1+t)^{1 / 2}}=\int_{0}^{H / j} \frac{j d \xi}{j(1+\xi)(1 / j+\xi)^{1 / 2} \cdot j^{1 / 2}}< \\
<j^{-1 / 2} \int_{0}^{\infty} \frac{d \xi}{(1+\xi) \xi^{1 / 2}}<4 j^{-1 / 2} .
\end{gathered}
$$

Now we combine inequalities (6.41)-(6.44) and come to

$$
(6.38) \leqslant \widetilde{C}\left(\omega(j)+\frac{\log (1+j)}{\sqrt{1+j}}\right) .
$$

Together with (6.37) it brings us to

$$
\left\|\frac{1}{2 \pi i} \int_{\Delta}\left(R_{\lambda}-R_{\lambda}^{0}\right) d \lambda\right\| \leqslant(6.45)_{\text {right }}(n)+(6.45)_{\text {right }}(k)+2(n+k)(n+k) H^{-3 / 2} \cdot m
$$

and with $H \rightarrow \infty$ we conclude that

$$
\left\|S_{k n}-S_{k n}^{0}\right\| \leqslant \varepsilon(k)+\varepsilon(n)
$$

where

$$
\varepsilon_{j}=\widetilde{C}\left(\omega(j)+\frac{\log (1+j)}{(1+j)^{1 / 2}}\right) .
$$

A part (6.23) of Theorem 6.1 has been proven!

4. Of course it immediately leads to a part (6.24). 
The decomposition (6.22) is orthogonal so for any $F \in \mathcal{H}$

$$
\lim _{\substack{k \rightarrow \infty \\ n \rightarrow \infty}} S_{k n}^{0} F=F
$$

but

$$
S_{k n}=S_{k n}^{0}+T_{k n}
$$

where

$$
T_{k n}=S_{k n}-S_{k n}^{0}
$$

and by (6.23)

$$
\left\|T_{k n}\right\| \leqslant \varepsilon(k)+\varepsilon(n) .
$$

But $\varepsilon(j) \searrow 0(j \rightarrow \infty)$, therefore (6.49)-(6.52) imply (6.24).

Theorem 6.1 is proven. i.e.

5. Trigonometric Fourier series (6.21) converges in $A L L L^{p}$-spaces, $1<p<\infty$,

$$
\left\|S_{k n}^{0} F-F\right\|_{p} \rightarrow 0, \quad \forall F \in L^{p},
$$

so it is natural to ask (and prove!) whether an analogue of (6.23) holds in $L^{p}$, $1<p<\infty$. We'll present related results under general assumption $V \in L^{b}, 1<b$, $V \in K(m, M)$.

THEOREM 6.2. Under the assumptions and notations of Theorem 6.1,

$$
\begin{gathered}
\text { (a) } \quad \text { if } b>\frac{4}{3}, \quad 1<a \leqslant 2 \leqslant c<\infty, \\
\text { (b) if } a<b \leqslant 2, \quad \frac{1}{a}+\frac{1}{b}-\frac{1}{c}<1, \\
\left(b^{\prime}\right) \quad \text { if } 2 \geqslant a \geqslant b, \quad \frac{2}{b}-\frac{1}{c}<1,
\end{gathered}
$$

there exists a sequence $\left\{\varepsilon_{n}>0\right\}, \varepsilon_{n} \searrow 0(n \rightarrow \infty)$ depending on $\{\Omega\}$ and $a, b, c$, such that

$$
\left\|\left(S_{k n}-S_{k n}^{0}\right): L^{a} \rightarrow L^{c}\right\| \leqslant \varepsilon(k)+\varepsilon(n) .
$$

Proof. Proof follows the same lines as the proof of Theorem 6.1. We choose a contour $\Delta \in(6.30), k, n \geqslant N, N$ and $H$ large enough so

$$
\left(S_{k n}-S_{k n}^{0}\right)=\frac{1}{2 \pi i} \int_{\Delta}\left(R_{\lambda}-R_{\lambda}^{0}\right) d \lambda .
$$

On horizonthal sides $\gamma^{ \pm} \in(6.32)$ we use a representation

$$
R_{\lambda}-R_{\lambda}^{0}=R_{\lambda}^{0} V R_{\lambda}^{0}\left(I-V R_{\lambda}^{0}\right)^{-1} .
$$

By Lemma 5.3, inequality (5.22) for

$$
e=\min \{a, b\}
$$

and if (compare (4.65)-(4.68))

$$
|\operatorname{Im} \mu| \geqslant H \geqslant\left(C_{31} m\right)^{b /(b-1)}
$$


the norm in (6.61) is less than $\frac{1}{2}$. Therefore, with $H \in(6.62)$ and $\lambda \in \gamma^{ \pm}$

$$
\left\|\left(R_{\lambda}-R_{\lambda}^{0}\right): L^{a} \rightarrow L^{c}\right\| \leqslant 2 \cdot C_{35} A(e ; \mu) m A(\sigma ; \mu), \quad \frac{1}{\sigma}=\frac{1}{b}-\frac{1}{c}
$$

as it follows from Lemma 5.2, inequality (5.18), and the following factorization (compare (4.61), (4.62))

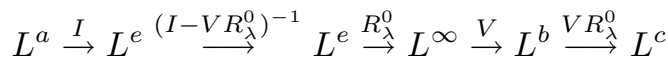

(where $I$ is an identity-embedding by (6.60)).

By inequality (3.17) the norm (6.63) does not exceed

$$
\begin{gathered}
C m \rho^{-(1-1 / e)} \cdot \rho^{-(1-1 / \sigma)}=C m \rho^{-\alpha}, \\
\alpha=2-\left(\frac{1}{b}+\frac{1}{e}-\frac{1}{c}\right) \geqslant 2\left(1-\frac{1}{e}\right)>0 .
\end{gathered}
$$

Finally,

$$
\int_{\gamma^{ \pm}}\left\|\left(R_{\lambda}-R_{\lambda}^{0}\right): L^{a} \rightarrow L^{c}\right\||d \lambda| \leqslant l\left(\gamma^{ \pm}\right) \cdot C H^{-\alpha},
$$

where $l\left(\gamma^{ \pm}\right)=n+k$. (Notice that this step (6.59)-(6.67) is possible if $c=\infty$ as well. We applied twice the inequality (5.18) with $\sigma=e>1$ and $1 / \sigma=1 / b-1 / c<1$ even if $c=\infty$.)

6. On the vertical sides (6.33), p. 165 we use a representation

$$
R_{\lambda}-R_{\lambda}^{0}=R_{\lambda}^{0} V R_{\lambda}^{0}\left(I+V R_{\lambda}^{0}\right)\left(I-V R_{\lambda}^{0} V R_{\lambda}^{0}\right)^{-1} .
$$

The estimates in Section 4.7 have been written for $a=b$; if $a<b$ we need first to analyze (in the same way) $K^{2}, K=V R_{\lambda}^{0}$ in $L^{e}$.

Case 1 . Choose $d$ by $\frac{1}{d}+\frac{1}{b}=\frac{1}{e}$. Then

$$
w^{*}=\left\|V R_{\lambda}^{0} V R_{\lambda}^{0}: L^{e} \rightarrow L^{d}\right\| \leqslant K_{1} A(\tau, \mu) A_{1}(b, \mu) M, \quad \frac{1}{\tau}=\frac{1}{e}-\frac{1}{d}=\frac{1}{b} .
$$

By the choice of $d$, and Lemma 3.1, inequality (3.21), Lemma 3.3, inequality (3.29)

$$
\begin{aligned}
\| V \cdot W: L^{e} & \rightarrow L^{e} \| \leqslant m M K_{2} \cdot \rho^{-(1-1 / b)}\left[\omega(n) \rho^{-(1-1 / b)}+\frac{n^{1 / b}}{n+\rho}\right] \leqslant \\
& \leqslant K_{3} m M\left[\omega(n)+n^{-(1-1 / b)}\right], \quad \rho \geqslant \frac{1}{4},
\end{aligned}
$$

and for $n \geqslant \widetilde{N}_{2}(m M)$ this norm $\leqslant 1 / 2$ so

$$
\left\|\left(I-V R_{\lambda}^{0} V R_{\lambda}^{0}\right)^{-1}: L^{e} \rightarrow L^{e}\right\| \leqslant 2 .
$$

The norm of second factor $I+V R_{\lambda}^{0}$ has been evaluated in (6.61); it does not exceed

$$
1+C m A(b ; \mu) \leqslant K_{4}<\infty .
$$

Therefore by (6.68) and (6.71), (6.72)

$$
\left\|\left(R_{\lambda}-R_{\lambda}^{0}\right): L^{e} \rightarrow L^{c}\right\| \leqslant 2 K_{4}\left\|R_{\lambda}^{0} V R_{\lambda}^{0}: L^{e} \rightarrow L^{c}\right\|
$$


and we can readily use Proposition 5.4, inequality (5.31) with $\{e, c\} \neq\{1, \infty\}$ by (6.60), and $1 / \tau=1 / e-1 / c$. It implies

$$
\beta=\left\|\left(R_{\lambda}-R_{\lambda}^{0}\right): L^{e} \rightarrow L^{a}\right\| \leqslant K M A(\tau ; \mu) A_{1}(b ; \mu)
$$

and as in (6.69-(6.70), for $\rho \geqslant 1 / 5$,

$$
\leqslant M K \rho^{-(1-1 / c)}\left[\omega(n) \rho^{-(1-1 / b)}+\frac{n^{1 / b}}{n+\rho}\right] .
$$

Notice that

$$
\begin{aligned}
& \frac{1}{\tau}=\frac{1}{a}-\frac{1}{c} \quad \text { if } \quad a<b, \\
& \frac{1}{\tau}=\frac{1}{b}-\frac{1}{c} \quad \text { if } \quad a \geqslant b .
\end{aligned}
$$

Now we have the same $\alpha \in(6.65)$, i.e.

$$
-\left(1-\frac{1}{\tau}\right)-\left(1-\frac{1}{b}\right)=\left\{\begin{array}{cll}
-2+\frac{1}{a}+\frac{1}{b}-\frac{1}{c} & \text { if } \quad a<b, \\
-2+\frac{2}{b}-\frac{1}{c} & \text { if } \quad a \geqslant b .
\end{array}\right.
$$

Inequality (6.74) gives us the same estimates of pieces in (6.58) which come from vertical sides of $\Delta$. Indeed,

$$
\left\|\int_{\gamma_{j}^{ \pm}} \cdots\right\| K M \int_{1 / 2}^{H}\left[\omega(j) t^{-\alpha}+\frac{j^{1 / b} t^{-(1-1 / \tau)}}{j+t}\right] d t
$$

(compare with (6.41)-(6.45)). If (6.54)-(6.56) holds then $\alpha>1$ and

$$
\int_{1 / 2}^{H} t^{-\alpha} d t \leqslant \frac{2^{\alpha-1}}{\alpha-1}<\infty
$$

A second integral

$$
\int_{1 / 2}^{H} \frac{j^{1 / b} t^{-(1-1 / \tau)}}{j+t} d t \leqslant j^{1 / b-(1-1 / \tau)} \int_{0}^{H / j} \frac{d \xi}{(1+\xi) \xi^{1-1 / \tau}} \leqslant K j^{-\alpha+1} \rightarrow 0 \quad(j \rightarrow \infty) .
$$

Collect inequalities (6.67), (6.78) and (6.80) and we have (6.81)

$\left\|\left(S_{k n}-S_{k n}^{0}\right): L^{a} \rightarrow L^{c}\right\| \leqslant(n+k) C_{37} H^{-\alpha}+\widetilde{K} M\left(\omega(n)+\omega(k)+n^{-\alpha+1}+k^{-\alpha+1}\right)$.

if $H \rightarrow \infty$ we get

$$
\varepsilon(k)=\widetilde{K} M\left(\omega(k)+k^{-(\alpha-1)}\right) .
$$

Case 2 - i.e. Dir $\mathcal{H}^{-}$, or general Dir.

We have to repeat the same proof from (6.58) to (6.81) with one minor adjustment.

If $c<\infty$ in inequalities (6.73)-(6.74) we can use (5.33) of Proposition 5.4 instead if inequality (5.31). The right side in (5.33) has the same majorant (6.75), - just change constants $K$. So we can choose $\varepsilon(k)$ as $(6.82)$ with another constant $K$. instead of $\widetilde{K}$. It completes the proof of Theorem 6.2 
7. Some steps of the proof are valid even for $c=\infty$ but the second condition in (6.54) excludes $c=\infty$ anyway. Indeed if $1<a, b \leqslant 2$ then $1 / a+1 / b \geqslant 1 / 2+1 / 2=1$ and (6.55) cannot hold if $c=\infty$. This condition makes possible the estimates (6.78)-(6.79) and (6.80) with right sides independent on $H$ so we can go from (6.81) to (6.57) by sending $H$ to infinity. But we can try to choose a finite $H$ to get good estimates. This is a way to analyze uniform convergence, and we got through all the details in Section 7.

The condition (6.54) is written because the Young inequality (to both directions - see Lemma 5.1) has been applied in Proposition 5.4 in this range of parameters. But an obvious identity-embedding

$$
L^{d} \stackrel{I}{\rightarrow} L^{e}, \quad\|I\|=1 \quad \text { if } \quad d \geqslant e
$$

gives us an extension of inequality (6.57) to other $a$ and $c$, however, without improvement of the right side in (6.57) defined by (6.82) where

$$
\widetilde{K}=\widetilde{K}(a, b, c)<\infty
$$

if (6.54), (6.55) hold.

More accurately, for any $a, c$ let us define

$$
\underline{a}=\min \{a, 2\} \quad \text { and } \quad \bar{c}=\max \{c, 2\},
$$

then of course $L^{a} \stackrel{I}{\rightarrow} L^{\underline{a}}$ and $L^{\bar{c}} \stackrel{I}{\rightarrow} L^{c}$ and for any operator $T$

$$
\left\|T: L^{a} \rightarrow L^{c}\right\| \leqslant\left\|T: L^{\underline{a}} \rightarrow L^{\bar{c}}\right\| .
$$

If for example,

$$
a \geqslant 2 \text { and } c \leqslant 2
$$

we write (6.57) after (6.86) as

$$
\|\left(S_{k n}-S_{k n}^{0}: L^{a} \rightarrow L^{c}\|\leqslant\|\left(S_{k n}-S_{k n}^{0}: L^{2} \rightarrow L^{2} \| \leqslant \bar{\varepsilon}(k)+\bar{\varepsilon}(n)\right.\right.
$$

where by $(6.82)$

$$
\bar{\varepsilon}(j)=M \cdot \widetilde{K}(2, b, 2)\left(\omega(j)+j^{-(\bar{\alpha}-1)}\right)
$$

with $\bar{\alpha}$ coming from (6.77) or (6.65), where $e=\min (b, \bar{a})=b$, i.e.

$$
\bar{\alpha}=2-\left(\frac{1}{b}+\frac{1}{b}-\frac{1}{2}\right)=\frac{5}{2}-\frac{2}{b} .
$$

Condition (6.55) should hold but it takes the form

$$
\frac{1}{a_{*}}+\frac{1}{b}-\frac{1}{c^{*}}<1 \quad \text { if } \quad a_{*}=a<b
$$

and

$$
\frac{2}{b}-\frac{1}{c^{*}}<1 \quad \text { if } \quad a \geqslant b .
$$

In the case (6.87) it means

$$
\bar{\alpha}>1, \quad \text { or } \quad \frac{3}{2}-\frac{2}{b}>0, \quad \text { or } \quad b>\frac{4}{3} .
$$

Let us write explicitly the restriction (6.91) in two other cases

$$
\begin{array}{lll}
a \geqslant 2, \quad c \geqslant 2 & {\left[a_{*}=2, \quad c^{*}=c\right],} \\
a \leqslant 2, \quad c \leqslant 2, & {\left[a_{*}=a, \quad c^{*}=2\right] .}
\end{array}
$$


Therefore, we want (see (6.77))

$$
\frac{2}{b}-\frac{1}{c}<1, \quad \text { or } \quad \frac{1}{b}-\frac{1}{c}<\frac{1}{2}
$$

or

$$
\begin{gathered}
\frac{1}{c}+\frac{1}{b}-\frac{1}{2}<1, \quad \text { or } \quad \frac{1}{c}+\frac{1}{b}<\frac{3}{2} . \\
\frac{1}{a}+\frac{1}{b}<\frac{3}{2} \quad \text { if } \quad a \leqslant b
\end{gathered}
$$

and

$$
\frac{2}{b}<\frac{3}{2}, \quad \text { or } \quad b>\frac{4}{3} \quad \text { if } \quad b \leqslant a .
$$

These inequalities $(6.93),(6.96),(6.97)$ put restrictions on parameters in the cases $(6.87),(6.94),(6.95)$ correspondingly. But if they hold, the statement (6.57) is correct with $K<\infty$ and

$$
\varepsilon(k)=K\left(\omega(k)+k^{-\beta}\right), \quad \beta=\bar{\alpha}-1>0 .
$$

We do not write explicitly the observations and results of this subsection in the form of Proposition or Theorem but let us collect an arithmetic of this subsection into the following table which shows the value of $\beta=\bar{\alpha}-1$ in (6.100).

Table 6.3 for $\beta=\bar{\alpha}-1$

\begin{tabular}{|c|c|c|c|}
\hline & $a<b$ & $b \leqslant a \leqslant 2$ & $2 \leqslant a$ \\
\hline$c \geqslant 2$ & $1+\frac{1}{c}-\frac{1}{a}-\frac{1}{b}$ & $1+\frac{1}{c}-\frac{1}{b}$ & $1+\frac{1}{c}-\frac{2}{b}$ \\
\hline$c \leqslant 2$ & $\frac{3}{2}-\frac{1}{a}-\frac{1}{b}$ & $\frac{3}{2}-\frac{2}{b}$ & $\frac{3}{2}-\frac{2}{b}$ \\
\hline$c=a$ & $\frac{3}{2}-\frac{1}{a}-\frac{1}{b}$ & $\frac{3}{2}-\frac{2}{b}$ & $1+\frac{1}{a}-\frac{2}{b}$ \\
\hline
\end{tabular}

We have proven

Corollary 6.3. Under notations of Theorem 6.2, if $\beta$ of the Table 6.3 is positive then

$$
\left\|\left(S_{k n}-S_{k n}^{0}\right): L^{a} \rightarrow L^{c}\right\| \leqslant \varepsilon(k)+\varepsilon(n)
$$

where $\varepsilon$ is defined by (6.100) with $0<K_{58}<\infty$, i.e.

$$
\varepsilon(k)=K_{58}\left(\omega(k)+k^{-\beta}\right) .
$$

8. May be, it was not obvious why in Theorem 6.2 we have a condition $b>4 / 3$. Simple inequalities show that if $b \leqslant 4 / 3$, in $A L L$ six cases of the two first lines in Table 6.3 the set $\{(a, c) \mid \beta>0\}$ is empty. If $2 \geqslant b>\frac{4}{3}$, i.e. $\frac{1}{2} \leqslant \frac{1}{b}<\frac{3}{4}$, put $\delta=\frac{1}{b}-\frac{1}{2}$, the third line defines

$$
\left\{\frac{1}{a}: \beta>0 \text { for }(a, 0)\right)
$$

as an interval

$$
2 \delta<\frac{1}{a}<1-\delta
$$

In particular, if $b=2$ and $\delta=0$ all $a$ 's, $1<a<\infty$, lie in the interval (6.103). This observation leads us to the following statement. 
THEOREM 6.4. If a potential $V$ in (1.1) is a $L^{2}$-function then spectral expansion (6.21), or

$$
P_{*} F+\sum_{-\infty}^{\infty} P_{j} F=F, \quad \forall F \in L^{p},
$$

converges in $L^{p}, 1<p<\infty$.

Proof. For an individual $V \in L^{2}$ we can choose $\left\{\Omega_{k}\right\} \in(1.7)$ in such a way that $V \in H(\Omega) \equiv H(2, \Omega)$ defined in (1.6) of (5.10). This elementary fact is explained in detail in Section 8, Lemma 8.2. For $b=2$ as we observed just in the line after (6.103) $\beta>0$ for any $a=c, 1<a<\infty$. Then

$$
F-S_{k n} F=F-S_{k n}^{0} F+\left(S_{k n}^{0}-S_{k n}\right) F
$$

and by Corollary 6.3, inequation (6.101), $a=c=\rho$

$$
\left\|F-S_{k n} F\right\|_{p} \leqslant\left\|F-S_{k n}^{0} F\right\|_{p}+(\varepsilon(k)+\varepsilon(n))\|F\|_{p}
$$

with $\varepsilon(k) \rightarrow 0(k \rightarrow \infty)$.

But trigonometric Fourier series $S_{k n}^{0}$ converges in every $L^{p}, 1<p<\infty$, - see Zygmund [25], Ch.7, Thm. 6.4. Therefore, both terms on the right side (6.107) go to zero when $k, n \rightarrow \infty$. It proves Theorem 6.4.

REMARK. (Warning). All expansions we are talking about are defined by Riesz projections $P_{j}$, see Proposition 4.3, Section 4. In the case of Dirichlet-type $b c$ they are one-dimensional so putting aside a projector $P_{*}$ we have an eigenfunction expansion, i.e. (6.105) has a form

$$
F=P_{*} F+\sum_{|k|>N} f_{k} \Phi_{k}
$$

where

$$
L \Phi_{k}=\lambda_{k} \Phi_{k}, \quad \lambda_{k} \in D_{k} \in(4.86) .
$$

But in $\operatorname{Per}^{ \pm}$cases $\operatorname{dim} P_{j}=2$. Even if there are two eigenfunctions $\Phi, \Psi$ such that

$$
P_{j} F=\varphi_{j}(F) \Phi_{j}+\psi_{j}(F) \Psi_{j},
$$

$\varphi_{j}, \psi_{j}$ being coefficient linear functionals

$$
L \Phi_{k}=\lambda_{k} \Phi_{k}, \quad L \Psi_{k}=\lambda_{k}^{\prime} \Psi_{k}, \quad\left\{\lambda_{k}, \lambda_{k}^{\prime}\right\} \in(4.86)
$$

Theorem 6.4, or (6.105) does not tell us that we can split $P_{j}$ and sum (6.105) by adding one term from (6.110) at the time.In other words, we have no control over the norms of these linear functionals $\varphi_{j}, \psi_{j}$ although we can control even $L^{\infty}$-norms of eigenfunctions (see Thm. 8.4, (8.33) and Cor. 8.5, (8.45) in Section 8).

\section{Uniform Convergence of Spectral Decompositions}

Estimates of elementary functions $A, A_{1}, A_{2}$ (Sect 3) and the resolvents of Dirac operator (Sect 5 ) lead us to the series of further results on convergence of spectral expensions. In this analysis and in the proofs we sometime need to repeat routine developed in the previous sections, in particular, our basic scheme in the estimates of the norm of $R_{\lambda}^{0} V R_{\lambda}^{0}$ from the proof of Proposition 2.4; see (2.44)) and (2.60)(2.61). 
1. First, we explain that the system of Riesz projectors is complete; more precisely, the following is true.

Proposition 7.1. Let

$$
V \in L^{b}, b>1,
$$

and $b c=P e r^{ \pm}$or Dir. Put

$$
E_{k}=\operatorname{Im} P_{k},|k|>N, \quad E_{*}=\operatorname{Im} P_{*} ;
$$

then the linear span

$$
E=\bigcup_{m=N+1}^{\infty} \mathbb{E}_{m}, \quad \mathbb{E}_{m}=\operatorname{Span}\left\{E_{*} ; E_{k}, N<|k| \leq m\right\}
$$

is dense in the space $C_{b c}([0,1])$ of continuous functions with $b c=P e r^{ \pm}$, Dir.

Proof. If $V=0$, then we know the systems of eigenfunctions (1.4), (1.5) for each $b c$. These systems $\left\{f_{j}\right\}$ are complete, i.e., the proposition's claim holds. Notice that

$$
S_{n} f_{j} \in \mathbb{E}_{n}, \quad n>N, \quad \forall j .
$$

The general case will be done if we show that

$$
\left\|S_{n} f_{j}-f_{j}\right\| \rightarrow 0 \quad \text { as } n \rightarrow \infty \quad \forall j
$$

where

$$
S_{n}=S_{n n}, \quad S_{n}^{0}=S_{n n}^{0}
$$

of (6.21), (6.22). For $n>2 j+1$

$$
S_{n}^{0} f_{j}=f_{j},
$$

so as in (6.58)

$$
S_{n} f_{j}-f_{j}=S_{n} f_{j}-S_{n}^{0} f_{j}=\frac{1}{2 \pi i} \int_{\Delta}\left(R_{\lambda}-R_{\lambda}^{0}\right) f_{j} d \lambda
$$

for a properly chosen countor $\Delta$. Now, we will use a second factorization in (2.2), i.e.,

$$
R_{\lambda}=\left(1-R_{\lambda}^{0} V\right)^{-1} R_{\lambda}^{0}=\left(1+\left(1-R_{\lambda}^{0} V\right)^{-1} R_{\lambda}^{0} V\right) R_{\lambda}^{0}
$$

so

$$
\begin{aligned}
& R_{\lambda}-R_{\lambda}^{0}=\left(1-R_{\lambda}^{0} V\right)^{-1} R_{\lambda}^{0} V R_{\lambda}^{0}= \\
= & \left(1-R_{\lambda}^{0} V R_{\lambda}^{0} V\right)^{-1}\left(1+R_{\lambda}^{0} V\right) R_{\lambda}^{0} V R_{\lambda}^{0} .
\end{aligned}
$$

Of course, as in (2.5) or (2.24), this formula (7.9) is valid if

$$
\lambda \notin \pi \mathbb{Z}, \quad\left\|R_{\lambda}^{0} V\right\|<1, \quad \text { or }\left\|\left(R_{\lambda}^{0} V\right)^{2}\right\|<1 .
$$

The advantage of this factorization is that for an individual vector $f$ the first factor acting on $f$ is the operator $R_{\lambda}^{0} V R_{\lambda}^{0}$. We have a special procedure to evaluate $R_{\lambda}^{0} V R_{\lambda}^{0} f$ if $f$ is a standard exponential - see the proof of Proposition 2.4.

We want to prove (7.4). Let us normalize $f$ 's so that $\left\|f_{j}\right\|_{2}=1, \forall j$; they are eigenvectors of $L_{b c}^{0}$. As in Sect 2 we have two cases:

(1) $b c=\operatorname{Per}^{ \pm}$or Dir for $\mathcal{H}^{+}$;

(2) Dir for $\mathcal{H}^{-}$. 
As in Sect $6.3,(6.30)-(6.33)$, we choose a counter $\Delta$ in (7.7) in the following way.

$$
\Delta=\partial \Pi_{n}(H),
$$

where

$$
\Delta=\partial \Pi_{n}(H), \quad \Pi_{n}(H)=\{z \in \mathbb{C}:|\operatorname{Re} z| \leq(n+1 / 2) \pi,|\operatorname{Im} z| \leq H\},
$$

so $\Delta$ is a union of six intervals

$$
\begin{gathered}
\gamma^{ \pm}=\left\{z \in \Pi_{n}(H): \quad \operatorname{Im} z= \pm H\right\} \\
\Gamma_{ \pm n}^{ \pm}=\left\{z \in \Pi_{n}(H): \operatorname{Re} z= \pm(n+1 / 2) \pi, 0 \leq \pm \operatorname{Im} z \leq H\right\} .
\end{gathered}
$$

By Lemma 4.4, (4.72), and Lemma 3.1, (3.21),

$$
\left\|R_{\lambda}^{0}: L^{b} \rightarrow L^{\infty}\right\| \leq A(b ; \mu) \leq C(b) \cdot\left(\frac{1}{|\operatorname{Im} \mu|+1}\right)^{1-1 / b} .
$$

Therefore, for $\lambda \in \gamma^{ \pm}$, we have $|\operatorname{Im} \mu|=H$, so

$$
\begin{gathered}
\left\|R_{\lambda}^{0} V: L^{\infty} \rightarrow L^{\infty}\right\| \leq m C(b) H^{1 / b-1} \\
\left\|R_{\lambda}^{0} V R_{\lambda}^{0}: L^{b} \rightarrow L^{\infty}\right\| \leq\left\|R_{\lambda}^{0}: L^{b} \rightarrow L^{\infty}\right\|^{2}\left\|V \mid L^{b}\right\|=m C^{2}(b) H^{2(1 / b-1)}
\end{gathered}
$$

and

$$
\left\|R_{\lambda}^{0} V R_{\lambda}^{0} V: L^{\infty} \rightarrow L^{\infty}\right\| \leq m^{2} C^{2} H^{2(1 / b-1)} .
$$

These inequalities guarantee that both lines in (7.9) are valid on $\gamma^{ \pm}$, with

$$
\left\|\left(1-R_{\lambda}^{0} V\right)^{-1}: L^{\infty} \rightarrow L^{\infty}\right\| \leq 2
$$

if $m C(b) H^{2(1 / b-1)} \leq 1 / 2$, and

$$
\left\|R_{\lambda}-R_{\lambda}^{0}: L^{b} \rightarrow L^{\infty}\right\| \leq 2 m C^{2}(b) H^{2(1 / b-1)}, \quad \lambda \in \gamma^{ \pm} .
$$

On intervals $\Gamma_{ \pm n}^{ \pm}$we use (7.9), the second line, and evaluate the norm of vectors

$$
R_{\lambda}^{0} V R_{\lambda}^{0} f_{j}
$$

see table in Sect 1.2, and (1.4), (1.5). As in Prop 2.4 we have two cases:

(1) $b c=\operatorname{Per}^{ \pm}$or Dir for $\mathcal{H}^{+}$;

(2) Dir for $\mathcal{H}^{-}$.

We follow the same scheme as in Prop 2.4; we'll use in the estimates only the assumption

$$
V \in L^{b}, \quad b>1, \quad m=\left\|V \mid L^{b}\right\|<\infty .
$$

Still we need the condition $V \in K(m, M) \in(5.3)$ to guarantee that $R_{\lambda}$ is welldefined on $\Gamma_{ \pm n}^{ \pm}$if

$$
\lambda=\pi n+\pi w, \quad|\operatorname{Re} w-1 / 2| \leq 1 / 3, \quad|n| \geq N_{*} .
$$

We use notations of the proof of Prop. 2.4.

Case 1. We consider a potential $V \in \mathcal{H}^{+}$and $b c=D i r$. If $b c=P e r^{ \pm}$and $V \in \mathcal{H}$ then the proof is the same. The decomposition (2.35), (2.38) holds in all $L^{p_{\text {-spaces, }}} 1 \leq p \leq \infty$, and by $(2.38)$

$$
\left\|V^{ \pm}\left|L^{p}\|\leq\| V\right| L^{p}\right\|
$$


Put $V=\sum g_{q} U_{q}^{+}$. By Lemma 5.1 and (7.0)

$$
\sum\left|g_{q}\right|^{b^{\prime}} \leq C^{\prime} m^{b^{\prime}}
$$

as in (2.44), with $\omega_{q}=1$,

$$
\begin{gathered}
u_{j} \stackrel{R_{\lambda}^{0}}{\longrightarrow} \frac{1}{\lambda-\pi j} u_{j} \stackrel{U_{q}^{+}}{\longrightarrow} \frac{1}{\lambda-\pi j} u_{q-j} \\
\stackrel{R_{\lambda}^{0}}{\longrightarrow} \frac{1}{\pi^{2}} \frac{1}{\mu-j} \frac{1}{m-(q-j)} u_{q-j},
\end{gathered}
$$

so

$$
R_{\lambda}^{0} V R_{\lambda}^{0} u_{j}=\frac{\alpha}{\mu-j} \sum_{q} \frac{g_{q}}{\mu-(q-j)} u_{q-j}, \quad \alpha=\frac{1}{\pi^{2}},
$$

and for $L^{\infty}$-norm $\nu_{j}$ of this vector we have by (2.12), and Lemma 3.1, (3.21), if $|\mu| \geq 1+2 j$

$$
\begin{array}{r}
\nu_{j}(\lambda) \leq \frac{\alpha}{|\mu-j|} \sum_{q} \frac{g_{q}}{|\mu-(q-j)|}\left\|u_{q-j}\right\|_{\infty} \\
\leq \frac{2 \alpha}{|\mu-j|}\left(\sum_{q}\left|g_{q}\right|^{b^{\prime}}\right)^{1 / b^{\prime}} \cdot A(b, \mu) \\
\leq \frac{4 \alpha m}{|n+w|} A(b ; w) \leq \frac{C m}{|n|+|m|+|v|} \cdot \frac{1}{1+|v|^{1-1 / b}} .
\end{array}
$$

Analogue of Prop 2.4 for $b, 1<b<2$, would give us - instead of (2.27) -

$$
\left\|R_{\lambda}^{0} V R_{\lambda}^{0}\right\|_{b \rightarrow \infty} \leq C(b) M A(b ; \mu) A_{1}^{b c}(b, \omega ; \mu),
$$

and therefore,

$$
\left\|R_{\lambda}^{0} V R_{\lambda}^{0}\right\|_{\infty \rightarrow \infty} \leq C(b) M m A(b ; \mu) A_{1}^{b c}(b, \omega ; \mu) .
$$

We will not use this inequality to estimate the norm of $R_{\lambda}-R_{\lambda}^{0}$ in (7.9), but (7.30) guarantees that the first factor in the second line is well defined and its norm in $L^{\infty}$ does not exceed 2 if $|n|>N_{*}(M)$, i.e., if the right side in (7.30) does not exceed $1 / 2$ in the strip

$$
Q_{n}=\{\lambda=\pi \mu=\pi(n+w):|\operatorname{Re} w-1 / 2| \leq 1 / 3\} .
$$

With (7.15)

$$
\left\|R_{\lambda}^{0} V: L^{\infty} \rightarrow L^{\infty}\right\| \leq C m(1+|v|)^{1 / b-1} \leq C m .
$$

Now we collect these inequalities (7.32), (7.28) and conclude by (7.9), that in the upper strip $S_{n},|n| \geq N_{*}$,

$$
\left\|\left(R_{\lambda}-R_{\lambda}^{0}\right) u_{j}\right\|_{\infty} \leq 2(1+C m) \frac{C m}{|n|+1+|v|} \cdot \frac{1}{1+|v|^{1-1 / b}} .
$$

Recall that $|n| \geq 1+2 j$. We succeeded to get (7.33) on $\Gamma_{ \pm n}^{ \pm}$. For $\gamma^{ \pm}$we have a simpler estimate (7.21). Finally, by (7.7)

$$
\begin{gathered}
S_{n} u_{j}-u_{j}=\frac{1}{2 \pi i} \int_{\Delta}\left(R_{\lambda}-R_{\lambda}^{0}\right) u_{j} d \lambda= \\
\frac{1}{2 \pi i}\left(\int_{\gamma}^{+}+\int_{\gamma}^{-}+\int_{\Gamma_{+n}^{+}}+\int_{\Gamma_{-n}^{+}}+\int_{\Gamma_{+n}^{-}}+\int_{\Gamma_{-n}^{-}}\right)
\end{gathered}
$$


with proper orientations of these six intervals. By (7.21) for fixed $n \geq N_{*}(M)$ with length $\gamma^{ \pm}=2 n$

$$
\left\|\frac{1}{2 \pi i} \int_{\gamma^{ \pm}}\right\| \leq C(b) m H^{2(1 / b-1)} \cdot 2 n .
$$

If we choose

$$
H=n^{r}, \quad 1>\frac{1}{2 r}+\frac{1}{b}, \text { i.e. } r>\frac{b}{2(b-1)},
$$

then

$$
H^{2(1 / b-1)} \rightarrow 0 \quad(n \rightarrow \infty),
$$

and

$$
\left\|\int_{\gamma^{ \pm}}\right\| \rightarrow 0 \quad(\rightarrow \infty) .
$$

Four remaining integrals have the same upper estimate by (7.33), with $\beta=1-1 / b$,

$$
\|\| \int_{\Gamma_{+n}^{+}} \| \leq C(1+m)^{2} \varepsilon_{n}, \quad \varepsilon_{n}=\int_{0}^{\infty} \frac{1}{1+v^{\beta}} \cdot \frac{d v}{n+v} .
$$

We omit an elementary exercise to explain that with $1>\beta>0$

$$
\varepsilon_{n} \rightarrow 0 \quad(n \rightarrow \infty)
$$

more precisely,

$$
\lim n^{\beta} \varepsilon_{n}=D(\beta)=\int_{0}^{\infty} \frac{d \xi}{\xi^{\beta}(1+\xi)}<\infty .
$$

By (7.34), the limits (7.38), (7.40) guarantee that

$$
\lim \left\|S_{n} f_{j}-f_{j}\right\|=0, \quad \forall j .
$$

Therefore, $f_{j} \in \bar{E}, \quad E \in(7.3)$ and

$$
\bar{E} \supset \overline{\operatorname{LinSpan}\left(f_{j}\right)}=C_{b c}[0,1] .
$$

Proposition 7.1 is proven in Case 1.

Case 2. $V \in \mathcal{H}, b c=$ Dir.

We follow the same scheme. The formulas (7.21) and (7.38) hold anyway. But (7.25) - (7.28) need to be adjusted and substituted by analogue of (2.60)-(2.61) instead of (2.44).

Put

$$
V=\sum_{q \text { even }} g_{q} U_{q}^{-} \in L^{b} \cap \mathcal{H}^{-}
$$

As in (7.24)

$$
\sum\left|g_{q}\right|^{b^{\prime}} \leq(2 m)^{b^{\prime}}
$$

By $(2.60)$, with $\omega_{q}=1$,

$$
u_{j} \stackrel{R_{\lambda}^{0}}{\longrightarrow} \frac{1}{\lambda-\pi j} u_{j} \stackrel{U_{q}^{-}}{\longrightarrow} \frac{1}{\lambda-\pi j} v_{q-j}=\frac{1}{\lambda-\pi j} \frac{i}{\pi} \sum_{\ell+j \text { odd }} \frac{1}{q-j-\ell} u_{\ell}
$$




$$
\stackrel{R_{\lambda}^{0}}{\longrightarrow} \frac{i}{\pi^{2}} \frac{1}{\mu-j} \sum_{\ell+j \text { odd }} \frac{1}{q-j-\ell} \cdot \frac{1}{\mu-\ell} u_{\ell}
$$

and instead of (7.26) we have, with $\alpha=\frac{i}{\pi^{2}}$,

$$
R_{\lambda}^{0} V R_{\lambda}^{0} u_{j}=\frac{\alpha}{\mu-j} \sum_{q} \sum_{\ell+j \text { odd }} \frac{1}{q-j-\ell} \cdot \frac{1}{\mu-\ell} u_{\ell} .
$$

Let $\nu_{j}$ be $L^{\infty}$-norm of this vector (7.46), and introduce a function

$$
B^{*}(s)=\sum_{k \in \mathbb{Z} \backslash\{0\}}\left|\frac{1}{k(k+s)}\right|, \quad s \notin \mathbb{Z},
$$

which is similar to the function $B$ in (2.75) and (3.30).

LEMMA 7.2. If

$$
|s-\rho| \geq 1 / 4, \quad \forall p \in \mathbb{Z},
$$

then

$$
B^{*}(s) \leq D \frac{1+\log (|s|+1)}{1+|s|}
$$

where $D$ is an absolute constant.

Proof is an analogue of the proof of Lemma 3.4, and we omit technical details. Again, if

$$
|\mu| \geq 1+2 j
$$

then

$$
\frac{1}{2}(1+|\mu|) \leq|\mu \pm j| \leq 2|\mu| .
$$

By (7.46) and (2.12)

$$
\nu_{j} \leq \frac{C}{|\mu|}\left(\sum\left|g_{q}\right|^{b^{\prime}}\right)^{1 / b^{\prime}} \cdot\left(\sum_{q}\left(B^{*}(\mu+j-q)\right)^{b}\right)^{1 / b} .
$$

By Lemma 7.2

$$
B^{*}(\mu+j-q) \leq D_{2} \frac{1+\log (1+|\mu+j-q|}{1+|\mu+j-q|} .
$$

With

$$
\mu=n+w, \quad 1 / 4 \leq|\operatorname{Re} w| \leq 3 / 4
$$

this inequality (7.53) implies that

$$
\sum_{q}\left(B^{*}(\mu+j-q)\right)^{b} \leq D_{3} \sum_{k}\left(\frac{1+\log (1+|\mu|+k \mid)}{(1+|w|+k \mid)^{b-1}}\right)^{b}
$$

Therefore, in (7.52) we have by Lemma 3.6

$$
\nu_{j}(n) \leq \frac{D(j)}{1+|n|+|w|} \cdot \frac{1+\log (1+|w|)}{(1+|w|)^{1-1 / b}} .
$$


This is an anlogue of (7.33). Now we can repeat the scheme (7.34-7.42); the only adjustment that is required is (7.39). We have

$$
\begin{gathered}
\left\|\int_{\Gamma_{ \pm n}^{ \pm}} \cdots\right\| \leq C\left(1+m^{2}\right) \varepsilon_{n}^{\prime}, \\
\varepsilon_{n}^{\prime}=\int_{0}^{\infty} \frac{d v}{n+v} \cdot \frac{\log (1+v)}{(1+v)^{1-1 / b}} .
\end{gathered}
$$

Of course,

$$
\lim _{n \rightarrow \infty} \varepsilon_{n}^{\prime}=0 \quad \text { if } b>1 .
$$

This completes the proof of the claim (comp. (7.42))

$$
\lim \left\|S_{n} u_{j}-u_{j}\right\|=0, \quad \forall j
$$

in Case 2. Proposition 7.1 is proven.

3. This propositions claim the completeness of the system of eigenvalues and associated vectors of Dirac operators for any potential $V \in L^{b}, b>1$. Now we'll show that if $V \in L^{2}$ then the partial sums

$$
S_{n} F=P_{*} F+\sum_{N_{*}<|k| \leq n} P_{k} F
$$

of a continuous vector-function $F \in C_{b c}[0,1]$ behave, roughly speaking, in the same way as partial sums of standard Fourier series, i.e., $S_{n}^{0} F$.

Proposition 7.3. If $V \in L^{2}$ and $V \in H(\Omega)$ with

$$
\text { (a) } \omega_{n} \log n \rightarrow 0 \quad(n \rightarrow \infty)
$$

or

$$
\text { (b) } \omega_{n}(\log n)^{2} \rightarrow 0 \quad(n \rightarrow \infty)
$$

then

$$
\sigma^{*}=\sup \left\|\left(S_{n}-S_{n}^{0}\right): L^{2} \rightarrow L^{\infty}\right\|<\infty
$$

(i) for $b c=P_{e r}^{ \pm}$and Dir if $V \in \mathcal{H}^{+}$and (a) holds;

(ii) for $b c=$ Dir if (b) holds.

Proof. Again we use the representation

$$
S_{n}-S_{n}^{0}=\frac{1}{2 \pi i} \int_{\Delta}\left(R_{\lambda}-R_{\lambda}^{0}\right) d \lambda
$$

with a countor $\Delta \in(7.11)-(7.14)$, and $R_{\lambda}-R_{\lambda}^{0}$ as in (7.9) or (6.7). Factoroperators in (7.9) or (6.7), besides of $R_{\lambda}^{0} V R_{\lambda}^{0}$, are uniformly bounded, and we need good estimates of the norms

$$
\nu(\lambda)=\left\|R_{\lambda}^{0} V R_{\lambda}^{0}: L^{2} \rightarrow L^{\infty}\right\|
$$

if we want to get (7.62). More precisely, say by (6.7),

$$
\left\|\left(R_{\lambda}-R_{\lambda}^{0}\right): L^{2} \rightarrow L^{\infty}\right\| \leq\left\|\left(1-V R_{\lambda}^{0}\right)^{-1}\right\|_{2 \rightarrow 2} \cdot \nu(\lambda)
$$

or

$$
\leq\left\|\left(1-R_{\lambda}^{0} V R_{\lambda}^{0}\right)^{-1}\right\|_{2}\left(1+\left\|V R_{\lambda}^{0}\right\|\right) \cdot \nu(\lambda) \leq 2(1+C m) \nu(\lambda) .
$$


In (7.35) we gave the estimate of an operator norm, not just the norm of a special vector. If $b=2$ we have

$$
\left\|\frac{1}{2 \pi i} \int_{\gamma^{ \pm}} \cdots\right\| \leq C(2) m H^{-1} n .
$$

On the intervals $\Gamma^{ \pm}$we use Prop. 5.4, (5.31) of (5.34), so

$$
\begin{aligned}
& \left\|R_{\lambda}^{0} V R_{\lambda}^{0}: L^{2} \rightarrow L^{\infty}\right\| \leq \\
& K A(2, \mu) A_{1}(2, \mu) \quad \text { if }(i), \\
& K A(2, \mu) A_{2}(2, \mu) \quad \text { if }(i i) .
\end{aligned}
$$

Under condition (7.31) for $\lambda=\pi \mu, \mu=n+w$,

$$
\begin{gathered}
A(2, \mu) \leq \frac{C}{(1+v)^{1 / 2}}, \quad|v|=|\operatorname{Im} \mu| \quad \text { (by Lemma 3.1, (3.22)); } \\
A_{1}(2, \mu) \leq C\left(\frac{\omega(n)}{(1+v)^{1 / 2}}+\frac{n^{1 / 2}}{n+v}\right)
\end{gathered}
$$

(by Lemma 3.3, (3.23) or (3.28));

$$
A_{2}(2, \mu) \leq C\left(\frac{\omega(n)}{(1+v)^{1 / 2}} \log (1+v)+\frac{n^{1 / 2} \log (1+n)}{n+v}\right)
$$

(by Lemma 3.7, (3.86)).

In the case (i) under the conditions (7.60) we evaluate $\left\|\int_{\Gamma^{ \pm}}\right\|$; Compare (7.39) and (15.2). We have by (7.69) and (7.72)-(7.73)

$$
\begin{array}{r}
\left\|\int_{\Gamma_{ \pm n}^{ \pm}} \cdots\right\| \leq C_{1}(m) \int_{\Gamma} \nu(\lambda)|d \lambda| \leq \\
\leq C_{2} \int_{0}^{H} \frac{1}{(1+v)^{1 / 2}}\left[\frac{\omega(n)}{(1+v)^{1 / 2}}+\frac{n^{1 / 2}}{n+v}\right] d v \\
\leq C_{3}\left[\omega(n)(\log H)+\int_{0}^{\infty} \frac{d \xi}{\xi^{1 / 2}(1+\xi)}\right]
\end{array}
$$

If we choose $H=n^{2}$, then the inequalities (7.67) and (7.75) together with (7.60) imply (7.62) in the case (i).

In the case (ii), even under the conditions (7.61), the direct analogue of the above argument with an inequality (7.74) would not work because the sequence $\int_{0}^{H} \frac{n^{1 / 2} \log n}{(1+v)^{1 / 2}(n+v)} d v$ is unbounded. But instead of using (7.74), or (3.86) in Lemma 3.7, let us incorporate the condition (7.61) into the estimates of $a(\mu)=A_{2}(2, \omega ; \mu) \in$ (3.4) and accordingly adjust (3.86). We have for $\lambda \in Q_{n} \in(7.31)$

$$
\begin{gathered}
a(\mu)=\left[\sum_{m \text { even }} \omega^{2}(m)\left[\frac{\log (2+2|2 n-m|)}{(1+|2 n-m+2 w|)}\right]^{2}\right]^{1 / 2} \\
\leq C\left[\sum_{m \text { even }} \tilde{\omega}^{2}(m)\left[\frac{\log (2+2|2 n-m|)}{(1+|2 n-m|+2|w|)(1+\log (2+|m|)}\right]^{2}\right]^{1 / 2},
\end{gathered}
$$


where

$$
\tilde{\omega}(m)=\max _{|k| \geq|m|} \omega(k)(1+\log (2+|k|)) \leq C_{3} /(1+\log (2+|m|))
$$

by (7.61). As in the proof of Lemma 3.5, the sum $\sigma$ on the right side in (7.76) can be estimated in the following way (assume $n>0$ large enough)

$$
\sum \leq \sum_{m \leq n}+\sum_{m \geq n}
$$

with

$$
\begin{gathered}
\sum_{m \geq n} \leq \tilde{\omega}^{2}(n) \cdot D_{1}(\mu), \\
D_{1}(\mu)=\sum_{k \geq 0}\left[\frac{\log (2+2 k)}{(1+k+v) \log (2+|2 n-k|)}\right]^{2},
\end{gathered}
$$

and

$$
\sum_{k \geq n} \leq D_{2}(\mu)=\sum_{k \geq 0}\left[\frac{\log (2+2 k)}{(1+k+n+v) \log (2+|2 n-k|)}\right]^{2} .
$$

Let us do $D_{2}$ first. With $v$ in the interval $[0, H], H \leq 3 n$ and $k \geq n$

$$
1+k \leq 1+k+n+v \leq 1+k+4 n \leq 1+5 k,
$$

and

$$
|2 n-k| \geq k / 3 \quad \text { if } k \geq 3 n .
$$

Under the condition (7.83)

$$
\frac{\log (2+2 k)}{1+\log (2+|2 n-k|)} \leq \frac{\log (2+2 k)}{1+\log (2+k / 3)} \leq C<\infty
$$

and this part of $D_{2}$

$$
\sum_{k \geq 3 n} \leq C \sum_{k \geq 3 n} \frac{1}{(1+k)^{2}} \leq C / n .
$$

If $n \leq k \leq 3 n$ then the factor (7.82) is between $1+2 n$ and $1+5 n$ when $0 \leq v \leq H$; therefore $[n, 3 n]$-part of $D_{2}$

$$
\sum_{n \leq k \leq 3 n} \leq C \frac{\log ^{2}(2+2 n)}{(1+2 n)^{2}} \sum_{n}^{3 n}\left(1+\log (2+|2 n-k|)^{-2} .\right.
$$

The second factor $D_{2}^{\prime}$ can be evaluated as

$$
\begin{gathered}
D_{2}^{\prime} \leq C \int_{n}^{3 n} \frac{d x}{[1+\log (2+|2 n-x|)]^{2}} \leq \\
\leq 2 C \int_{n}^{3 n} \frac{d x}{[1+\log (2+2 x)]^{2}}=2 C \int_{0}^{1} \frac{n d \xi}{[1+\log (2+2 n x)]^{2}} \\
\leq 2 C n\left(\int_{0}^{n^{-1 / 8}}+\int_{n^{-1 / 8}}^{1}\right) \leq \tilde{C}\left[n^{7 / 8}+\frac{n}{(1+\log (2+n))^{2}}\right] .
\end{gathered}
$$


The last term comes from an observation

$$
0<\inf _{n \geq 0} \frac{\log \left(2+2 n^{7 / 8}\right)}{\log (2+n)} \leq \sup _{n \geq 0} \frac{\log \left(2+2 n^{7 / 8}\right)}{\log (2+n)}<\infty
$$

with $\lim _{n \rightarrow \infty} \cdots=7 / 8$. Now, if we collect the inequalities (7.85), (7.86) and (7.87) we conclude that

$$
D_{2}(\mu) \leq C / n \text { if } 0 \leq v \leq 3 n .
$$

We still have to evaluate $D_{1}(\mu) \in(7.80)$. Subdivide $[0, \infty)$ into three intervals

$$
0 \leq k \leq n, \quad n<k \leq 3 n, \quad 3 n<k
$$

and accordingly the sum (7.80) into three parts

$$
d_{0}=\sum_{0}^{n}, \quad d_{1}=\sum_{n+1}^{3 n}, \quad d_{2}=\sum_{3 n+1}^{\infty} .
$$

Parts $d_{1}, d_{2}$ have been really done in analysis of $D_{2}$, and we got

$$
d_{1}+d_{2} \leq C / n \text {. }
$$

For $d_{0}$

$$
\log (2+|2 n-k|) \geq \log (2+n)
$$

so

$$
d_{0} \leq\left(\frac{\log (2+2 n)}{\log (2+n)}\right)^{2} \sum_{k=0}^{n} \frac{1}{(1+k+v)^{2}}
$$

and

$$
d_{0} \leq \frac{C}{1+v} .
$$

It follows from (7.92) and (7.95) that

$$
D_{1}(\mu) \leq C\left(\frac{1}{n}+\frac{1}{1+v}\right), \quad 0 \leq v \leq 3 n .
$$

After taking square root in the right side of (7.76), with (7.78), (7.96) and (7.89) we have

$$
a(\mu) \leq C\left(\tilde{\omega}(n) \cdot\left[\frac{1}{\sqrt{n}}+\frac{1}{\sqrt{1+v}}\right]+\frac{1}{\sqrt{n}}\right) .
$$

We have another factor $A(2, \mu)$ in $(7.68)$, so with $H=n$

$$
\begin{gathered}
\left\|\int_{\Gamma^{ \pm}}\right\| \leq C \int_{0}^{H} \frac{d v}{(1+v)^{1 / 2}} \cdot\left[\frac{\tilde{\omega}(n)}{\sqrt{1+v}}+\frac{1}{\sqrt{n}}\right] \\
\leq C\left[\tilde{\omega}(n) \log n+(1+H)^{1 / 2} n^{-1 / 2}\right]
\end{gathered}
$$

and (7.77) guarantees that with some constant independent on $n$ these integrals (7.98) are uniformly bounded. For intervals $\gamma^{ \pm}$the inequality (7.67) holds in the case (ii) as well. It completes the proof of the statement (7.62). Proposition 7.3 is proven. 
4. These results lead to our main statement in this section. Let us remind that F satisfies Dini condition if

$$
\int_{0}^{\pi} \frac{\tau(h)}{h} d h<\infty
$$

where $\tau$ is a modulus of continuity

$$
\tau(h)=\sup \left\{\left|F\left(x+h^{\prime}\right)-F(x)\right|:\left|h^{\prime}\right| \leq h\right\}, \quad 0<h \leq \pi .
$$

TheOREm 7.4. Let $F$ satisfies Dini condition. Its spectral decomposition $P_{*} F+$ $\sum_{k>N} P_{k} F$ uniformly converges,i.e.,

$$
\lim _{n \rightarrow \infty}\left\|S_{n} F-F\right\|_{\infty}=0
$$

where

$$
S_{n} F=P_{*} F+\sum_{N<|k| \leq n} P_{k} F,
$$

if hypotheses if Proposition 7.3 holds, i.e., $V \in L^{2}$ and $V \in H(\Omega)$, and

$$
\text { (a) } \omega(n) \log n \rightarrow 0
$$

if $b c=P_{e r}^{ \pm}$, or Dir with $V$ "even", i.e., $q(x)=p(-x)$;

$$
\text { (b) } \omega(n)(\log n)^{2} \rightarrow 0
$$

if $b c=$ Dir with any $V$.

Proof. Notice, that for any "polynomial" $G$, i.e., with $\left\{u_{k}\right\}$ being complete o.n.s. of eigenfunctions for $L_{b c}^{0}$ (see Table, Sect. 1.2),

$$
G=\sum_{k \in K} \xi_{k} u_{k}, \quad \# K<\infty,
$$

we have

$$
S_{n} F-F=\left(S_{n}^{0} F-F\right)+\left(S_{n}-S_{n}^{0}\right) G+\left(S_{n}-S_{n}^{0}\right)(F-G) .
$$

By Proposition 7.1 we know that "polynomials" are dense in $C_{b c}([0,1])$, but we need only to know that polynomials are dense in $L^{2}$ to choose for any $\delta>0$ such $G \in(7.105)$ that

$$
\|F-G\|<\delta
$$

and by (7.62) of Proposition 7.3

$$
\left\|\left(S_{n}-S_{n}^{0}\right)(F-G)\right\|_{\infty}<\sigma^{*} \cdot \delta, \quad \forall n \geq N_{*} .
$$

By (7.4) of Proposition 7.1

$$
\left\|\left(S_{n}-S_{n}^{0}\right) G\right\|_{\infty} \rightarrow 0 \quad(n \rightarrow \infty) .
$$

For standard Fourier series, Dini condition guarantees uniform convergence (see [25], Sect. 2.6, Thm. 6.8), so

$$
\left\|S_{n}^{0} F-F\right\|_{\infty} \rightarrow 0 \quad(n \rightarrow \infty) .
$$

These claims (7.110), (7.109) and (7.108) imply for (7.106)

$$
\limsup \left\|S_{n} F-F\right\|_{\infty} \leq \sigma^{*} \cdot \delta \text {. }
$$

But $\delta$ in (7.107) could be any positive number, so by (7.111)

$$
S_{n} F \rightarrow F \quad \text { uniformly, }
$$


i.e., in $C_{b c}([0,1])$. Theorem 7.4 is proven.

\section{8. $L^{2}$-Unconditional Convergence; Uniform Boundedness of Eigenfunctions}

In the previous section the estimates of operator norms of $R_{\lambda}^{0} V R_{\lambda}^{0}$ on the boundary of large rectangles $\Pi \in(7.12)$ helped us to analyze partial sums

$$
S_{n} F=P_{*} F+\sum_{N<|k| \leq n} P_{k} F
$$

of the spectral decomposition and prove a series of results on uniform convergence. But analysis of individual projectors $P_{k}$ could be quite useful and informative as well.

First, we state the following.

Proposition 8.1. Let $V \in L^{b}, 1<b$ and $1<a$. For $b c=$ Per $^{ \pm}$, Dir, there exists $\delta_{n} \rightarrow 0$ depending on $V$ such that

$$
\left\|\left(P_{n}-P_{n}^{0}\right): L^{a} \rightarrow L^{\infty}\right\| \leq \delta_{n}, \quad n \geq N_{*} .
$$

Proof. Assume for a while that

$$
V \in H(b ; \Omega), \quad V \in K(m, M) \quad(\text { see }(5.13))
$$

with $\Omega \in(1.7)$. As in (7.7)

$$
P_{n}-P_{n}^{0}=\frac{1}{2 \pi i} \int_{D_{n}}\left(R_{\lambda}-R_{\lambda}^{0}\right) d \lambda
$$

with

$$
D_{n}=\{z \in \mathbb{C}: z=\pi(n+w),|w|=1 / 4\} .
$$

Again, we use a representation

$$
R_{\lambda}-R_{\lambda}^{0}=R_{\lambda}^{0} V R_{\lambda}^{0}\left(1+V R_{\lambda}^{0}\right)\left(1-V R_{\lambda}^{0} V R_{\lambda}^{0}\right)^{-1} .
$$

For $|n|>N$ the first and second factors from the right have uniform estimates of their norms in $L^{a}$ on $\partial D_{n}$, i.e.,

$$
\left\|\left(1+V R_{\lambda}^{0}\right)\right\|_{a}, \quad\left\|\left(1-V R_{\lambda}^{0} V R_{\lambda}^{0}\right)^{-1}\right\|_{a} \leq C, \quad \lambda \in \partial D_{n},|n|>N,
$$

where $C$ depends on $a, b, \Omega$ but not on $n$ and $\lambda$. Therefore, by (8.6) and (8.7),

$$
\|\left(R_{\lambda}-R_{\lambda}^{0}: L^{a} \rightarrow L^{\infty}\|\leq C\| R_{\lambda}^{0} V R_{\lambda}^{0}: L^{a} \rightarrow L^{\infty} \| \leq C w^{*},\right.
$$

where by Proposition 5.4

$$
w^{*} \leq K A(a, \mu) A_{1}(b, \mu) \quad \text { in the Case } 1,
$$

or

$$
w^{*} \leq K A(a, \mu) A_{2}(b, \mu) \quad \text { in the Case } 2 .
$$

By Lemma 3.1 [put $\rho=1 / 4$ there]

$$
A(a ; \mu) \leq C(a) \quad \text { if } \lambda \in \bigcup_{|k|>N} \partial D_{k} .
$$

The second factor in (8.10) or (8.11) is evaluated in Lemma 3.3, (3.23), or Lemma $3.5,(3.47)$. We have

$$
A_{1}(b, \mu) \leq C\left[\omega(n)+n^{1 / b-1}\right],
$$


or

$$
A_{2}(b, \mu) \leq C\left[\omega(n)+n^{1 / b-1} \log n\right] .
$$

In either case [with $\log n \geq 1$ for $n>N$ ] it implies by (8.8)

$$
w^{*} \leq C\left[\omega(n)+n^{1 / b-1} \log n\right]
$$

and by $(8.4)$

$$
\left\|\left(P_{n}-P_{n}^{0}\right): L^{a} \rightarrow L^{\infty}\right\| \leq C\left[\omega(n)+n^{1 / b-1} \log n\right] \rightarrow 0 .
$$

To complete the proof, we need to explain that the assumption $V \in H(b, \Omega)$ is not restrictive. Indeed, the following is true.

Lemma 8.2. Let $Q$ be a compact set in $L^{b}, 1<b$. Then, a sequence $\Omega \in(1.7)$ could be chosen in such a way that

$$
Q \subset H(b, \Omega) \quad \text { and } Q \in K(m, M) \text { for some } m, M>0 .
$$

Proof. Of course, we can choose

$$
m=\sup \left\{\|V\|_{b}: V \in Q\right\}<\infty .
$$

By Hausdorff-Young inequality [Lemma 5.1]

$$
\sum\left(\left|p_{k}\right|^{\beta}+\left|q_{k}\right|^{\beta}\right) \leq 2\|V\|_{b}^{\beta}, \quad V=\left(\begin{array}{cc}
0 & p \\
q & 0
\end{array}\right), \quad \frac{1}{b}+\frac{1}{\beta}=1 .
$$

These series converge uniformly (!) on a compact $Q$ so with a notation

$$
\left(\tau_{n}(V)\right)^{2}=\sum_{|k| \geq n}\left(\left|p_{k}\right|^{\beta}+\left|q_{k}\right|^{\beta}\right)
$$

we define

$$
\tau_{n}=\sup \left\{\tau_{n}(V)+e^{-n} \mid V \in Q\right\} .
$$

[We include an artificial term $e^{-n}$ to avoid an unnecessary talk about possible zeroes.] This is a positive sequence

$$
\tau_{n}>0, \quad \tau_{n} \downarrow 0(n \rightarrow \infty), \quad 0<\tau_{0} \leq \sqrt{2} m^{\beta / 2}+1 .
$$

Put

$$
\Omega(-n)=\Omega(n)=\left(\tau_{0} / \tau_{n}\right)^{1 / \beta}, \quad n \geq 0 .
$$

Then for $V \in Q$

$$
\begin{gathered}
\sum \Omega^{\beta}(k)\left(\left|p_{k}\right|^{\beta}+\left|q_{k}\right|^{\beta}\right)=\tau_{0} \sum \frac{\left|p_{k}\right|^{\beta}+\left|q_{k}\right|^{\beta}}{\tau_{k}} \leq \\
\leq \tau_{0} \sum_{n \geq 0} \frac{\tau_{n}(V)^{2}-\tau_{n+1}(V)^{2}}{\tau_{n}} \\
\leq 2 \tau_{0} \sum_{n \geq 0} \frac{\tau_{n}(V)^{2}-\tau_{n+1}(V)^{2}}{\tau_{n}(V)+\tau_{n+1}(V)} \cdot \frac{\tau_{n}(V)+\tau_{n+1}(V)}{\tau_{n}+\tau_{n+1}} \\
\leq 2 \tau_{0} \sum_{n \geq 0}\left(\tau_{n}(V)-\tau_{n+1}(V)\right) \leq 2 \tau_{0}^{2} .
\end{gathered}
$$

Therefore,

$$
Q \in K(m, M), \quad \text { with } M=\left(2 \tau_{0}^{2}\right)^{1 / \beta} \leq\left[4\left(2 m^{1 / \beta}+1\right)\right]^{1 / \beta} .
$$

Lemma 8.2 is proven. 
Now we can apply (8.15), and choose

$$
\delta_{n}=C\left[\omega(n)+(1+n)^{-1 / \beta} \log (1+n)\right], \quad n \geq 0 .
$$

Proposition 8.1 is proven.

By duality it implies the following

Proposition 8.3. Under the conditions of Proposition $8.1\left(\alpha=a^{\prime}\right)$

$$
\left\|\left(P_{n}-P_{n}^{0}\right): L^{1} \rightarrow L^{\alpha}\right\| \leq \delta_{n}, \quad n \geq N_{*} .
$$

Proof. By duality (8.2) of Proposition 8.1 implies that for any potential in $L^{b}, 1<b$,

$$
\left\|\left(P_{n}-P_{n}^{0}\right)^{*}: L^{1} \rightarrow L^{\alpha}\right\| \leq \delta_{n} .
$$

But

$$
P_{n}=P_{n}(V)=\frac{1}{2 \pi i} \int_{\partial D_{n}} R_{\lambda}(V) d \lambda
$$

so

$$
P_{n}^{*}=-\frac{1}{2 \pi i} \int_{\partial D_{n}} R_{\bar{\lambda}}(V) d \bar{\lambda}=\frac{1}{2 \pi i} \int_{\partial D_{n}} R_{z}(V) d z=P_{n}\left(V^{*}\right)
$$

where

$$
V^{*} F=\left[\begin{array}{cc}
0 & \overline{q(x)} \\
\overline{p(x)} & 0
\end{array}\right]\left(\begin{array}{l}
f_{1} \\
f_{2}
\end{array}\right)=\left[\begin{array}{c}
\overline{q(x)} f_{2}(x) \\
\overline{p(x)} f_{1}(x)
\end{array}\right] .
$$

Therefore,

$$
P_{n}(V)^{*}=P_{n}\left(V^{*}\right), \quad P_{n}(V)=P_{n}\left(V^{*}\right)^{*} .
$$

Of course, $\left(P_{n}^{0}\right)^{*}=P_{n}^{0}$ in $L^{2}$ and on polynomials; it does not depend on $V$ so

$$
\left(P_{n}\left(V^{*}\right)-P_{n}^{0}\right)^{*}=P_{n}(V)-P_{n}^{0} .
$$

A change $V \rightarrow V^{*}$ in (8.30) does not change $L^{b}$-norm of the potential so (8.2) of Proposition 8.1 written for a potential $V^{*}$ implies (8.27) for the potential $V$. Proposition 8.3 is proven.

Now we are ready to prove the uniform $L^{\infty}$-boundedness of $L^{2}$-normalized eigenfunctions of $L_{b c}$. More precisely, the following holds.

TheOREM 8.4. Let $V \in L^{b}, 1<b$, and $V \in K(m, M)$. Let $\left\{P_{*} ; P_{n}, n \geq N\right\}$ be the projectors defined in Proposition 4.6. Then for some $B>0$ depending only on $\Omega$ and $m, M$,

$$
\|F\|_{\infty} \leq B\|F\|_{1}, \quad \text { if } F \in \operatorname{Im} P_{n},|n|>N, \text { or } F \in \operatorname{Im} P_{*} .
$$

Proof. We'll use Propositions 8.1 and 8.3 with $a=2$ so

$$
\left\|\left(P_{n}-P_{n}^{0}\right): L^{2} \rightarrow L^{\infty}\right\| \leq \delta_{n} \rightarrow 0,
$$

and

$$
\left\|\left(P_{n}-P_{n}^{0}\right): L^{1} \rightarrow L^{2}\right\| \leq \delta_{n} .
$$

The structure of $P_{n}^{0}$, see Tables in Sect 2.2 and Proposition 4.3, guarantess that

$$
\left\|P_{n}^{0} F\right\|_{\infty} \leq 2\|F\|_{1}, \quad|n| \geq N
$$


and with $\operatorname{dim} P_{*}^{0} \leq 2 N+1$

$$
\left\|P_{*}^{0} F\right\|_{\infty} \leq 2(2 N+1)\|F\|_{1} .
$$

These observations show that (8.33) holds if

$$
V=0, \quad B^{0}=2(2 N+1) .
$$

Let $F \in \operatorname{Im} P_{n}$ and $\|F\|_{1}=1$. Then

$$
F=P_{n}^{0} F+\left(P_{n}-P_{n}^{0}\right) F
$$

and

$$
\|F\|_{2} \leq\left\|P_{n}^{0} F\right\|_{2}+\left\|\left(P_{n}-P_{n}^{0}\right): L^{1} \rightarrow L^{2}\right\| .
$$

By (8.36) and (8.35)

$$
\|F\|_{2} \leq 2+\delta_{n} \leq 2+\delta^{*}, \quad \delta^{*}=\sup _{|n| \geq N} \delta_{n} .
$$

If $F \in \operatorname{Im} P_{*}$ the same argument shows that

$$
\|F\|_{2} \leq\left\|P_{*}^{0} F\right\|_{2}+\left\|\left(P_{*}-P_{*}^{0}\right): L^{1} \rightarrow L^{2}\right\| \leq B^{0}+R,
$$

where

$$
R=\left\|\left(P_{*}-P_{*}^{0}\right): L^{1} \rightarrow L^{2}\right\| .
$$

With $B^{*}=B^{0}+2+\delta^{*}+R$ we have

$$
\|F\|_{2} \leq B^{*}\|F\|_{1}, \quad \text { if } F \in \operatorname{Im} P_{n}, n \geq N \text {, or } F \in \operatorname{Im} P_{*} \text {. }
$$

The same analysis, with (8.34) used instead of (8.35) leads to the following claim

$$
\|G\|_{\infty} \leq B^{*}\|G\|_{2} \quad \text { if } G \in \operatorname{Im} P_{n}, n \geq N, \text { or } G \in \operatorname{Im} P_{*} .
$$

The inequality (8.33) holds with $B=\left(B^{*}\right)^{2}$.

It should be pointed out that $R$ in (8.42) comes from the estimates of the integrals $\int_{\partial \Pi} \cdots$, see (6.25)-(6.30), and Thm 6.2, and it depends on $\Omega, m, M$ but not on an individual $V$ in $K(m, M)$.

Theorem 8.4 is proven.

COROLlary 8.5. If $\left\{f_{j}\right\}$ is a system of eigenfunctions of $L_{b c}, V \subset L^{b}, 1<b$, then for some constant $B$

$$
\left\|f_{j}\right\|_{\infty} \leq B\left\|f_{j}\right\|_{1}, \quad \forall j .
$$

Proof. As in Lemma 8.2 we can choose $\Omega$ and $m, M$ such that

$$
V \in K(m, M) \in(5.13) .
$$

For any $j f_{j} \in \operatorname{Im} P_{n}$ for some $n,|n|>N, f_{j} \in \operatorname{Im} P_{*}$. So, Theorem 8.4 implies (8.45). Corollary 8.5 is proven.

A special case of Proposition 8.1 with $a=2$ plays an important role in our analysis [P. Djakov and B. Mityagin, [5], Theorems 2,3] of smoothness of a complexvalued potential $V$ in terms of the decay rate of diameters of spectral triangles. We used it in (8.34) but let us state it as the following. 
Corollary 8.6. If $V \in L^{2}$ and $V \in H(\Omega)$ then for any $b c=P e r^{ \pm}$, Dir

$$
\left\|P_{n}-P_{n}^{0}: L^{2} \rightarrow L^{\infty}\right\| \leq C \delta_{n}, \quad|n|>N
$$

where

$$
\delta(-n)=\delta(n) \rightarrow 0 \quad(n \rightarrow \infty) .
$$

Moreover, we can choose

$$
\delta(n)=\omega(n)+(1+n)^{-1 / 2} \log (1+n), \quad|n|>N .
$$

This choice does not guarantee that $\sum \delta_{n}^{2}<\infty$ even if $\sum \omega(n)^{2}<\infty$. But if we consider norms

$$
\alpha_{n}=\left\|P_{n}-P_{n}^{0}: L^{2} \rightarrow L^{\infty}\right\|
$$

we can claim the following.

Proposition 8.7. If $V \in L^{2}, V \in H(\Omega)$ then

$$
\sum \alpha_{n}^{2} \leq C^{2} \sum \omega_{n}^{2}
$$

Proof. Again we use the representation

$$
P_{n}-P_{n}^{0}=\frac{1}{2 \pi i} \int_{\partial D_{n}}\left(R_{\lambda}-R_{\lambda}^{0}\right) d \lambda, \quad|n|>N
$$

with

$$
D_{n}=\{z=\pi(n+w):|w| \leq 1 / 4\},
$$

and Proposition 2.4. We get with (8.6)

$$
\left\|R_{\lambda}-R_{\lambda}^{0}\right\|_{2 \rightarrow 2} \leq 2(1+4 m)\left\|R_{\lambda}^{0} V R_{\lambda}^{0}\right\|_{2 \rightarrow 2},
$$

and by (2.27), Proposition 2.4, for $\lambda \in \partial D_{n}$

$$
\left\|R_{\lambda}^{0} V R_{\lambda}^{0}\right\|_{2 \rightarrow 2} \leq C M B_{n}
$$

where

$$
B_{n}^{2}=\sum_{p \text { even }} \omega_{p}^{2} \frac{\log ^{2}(3+|2 n-p|)}{1 / 16+(2 n-p)^{2}} .
$$

Of course, (8.52)-(8.55) imply

$$
\alpha_{n}=\left\|P_{n}-P_{n}^{0}\right\|_{2 \rightarrow 2} \leq C(1+m) M B_{n} .
$$

But

$$
\sum B_{n}^{2}=\sum_{n} \sum_{p}=\sum_{p} \omega_{p}^{2} \sum_{n} \frac{\log ^{2}(3+|2 n-p|)}{1 / 16+(2 n-)^{2}}=\beta^{2} \sum_{p} \omega_{p}^{2},
$$

where

$$
\beta^{2} \leq 2 \sum_{k=0}^{\infty} \frac{\log ^{2}(3+2 k \mid)}{1 / 16+4 k^{2}}<\infty
$$

Therefore, by (8.57)-(8.59)

$$
\sum \alpha_{n}^{2} \leq \tilde{C}^{2} \sum \omega_{p}^{2}, \quad \tilde{C}=C(1+m) M \beta .
$$

Proposition 8.7 is proven. 
By A. Markus version $([\mathbf{1 6}, \mathbf{1 7}, \mathbf{7}])$ of N. Bari theorem on unconditional convergence we can go from Proposition 8.7 to the following.

THEOREM 8.8. If $V \in L^{2}$ and $V \in H(\Omega)$ with

$$
\sum \omega_{n}^{2}<\infty
$$

then the spectral decompositions

$$
F=P_{*}+\sum_{|n|>N} P_{n} F, \quad \forall F \in L^{2}
$$

converge unconditionally.

Proof. This decomposition (6.105) converges in $L^{2}$, according to Theorem 6.4. Then (8.61) and (8.51) of Proposition 8.7 imply

$$
\sum \alpha_{n}^{2}<\infty
$$

and by Bari-Markus Theorem, the series (8.62) converge in $L^{2}$ unconditionally. Theorem 8.8 is proven.

6. We conclude this section by a comment to Theorem 6.4 and condition (6.104). If in Cor. 6.3

$$
a=c, \quad 4 / 3<b \leq 2
$$

then (6.104) and (6.101) imply that spectral expansions (6.105) converge in $L^{a}$ for any $F \in L^{a}$ if

$$
2 \delta<1 / a<1-\delta, \quad 0 \leq \delta=1 / b-1 / 2<1 / 4 .
$$

This subinterval of $(0,1)$ is not symetric around $1 / 2$. However, the duality type argument we used in the proof of Proposition 8.3 could explain that if there is a convergence in (6.105) for $a, 1 / 2 \leq 1 / a<1-\delta, 0 \leq \delta<1 / 4$, then for $a^{\prime}$ such that

$$
\delta<1 / a^{\prime} \leq 1 / 2
$$

the spectral decompositions (6.105) converge in all $L^{a^{\prime}}$-spaces if (8.66) holds. It extends the interval $(2 \delta, 1-\delta)$ in $(8.65)$ to the interval $(\delta, 1-\delta)$. Therefore, the following refinement of Theorem 6.4 holds.

Proposition 8.9. If a potential $V \in L^{b}, 4 / 3 \leq b \leq 2$, then spectral expansions (6.21), or (6.105), converge in $L^{a}$,

$$
\delta \leq 1 / a \leq 1-\delta, \quad \delta=1 / b-1 / 2,
$$

for any $F \in L^{a}$ if $\delta>0$. 7.4 .

Remark. The case $\delta=0$, i.e., $b=2$, is covered by Theorem 6.4 and Theorem

Proof. In the case of strict inequality $1 / 2 \leq 1 / a<1-\delta$ we can repeat the argument in the proof of Theorem 6.4. If $1 / a=1-\delta$ we need to look carefully into the proof of Cor. 6.3 and realize that if $\beta=0$ then we can claim

$$
\left\|\left(S_{k n}-S_{k n}^{0}\right): L^{a} \rightarrow L^{a}\right\| \leq C(a)<\infty, \quad \forall k, n ; n \geq N .
$$

Then by the density theorem (Proposition 7.1) and convergence of standard Fourier series in $L^{p}$,

$$
S_{n}^{0} F \rightarrow F, \quad \forall F \in L^{p}, \quad 1<p<\infty,
$$


together with routine approximation theory scheme (see the proof of Theorem 7.4, (7.105)-(7.112)) guarantee that by (8.68) we have

$$
\lim S_{k n} F=F, \quad \forall F \in L^{a}, \quad a=\frac{1}{1-\delta}=\frac{3 b-2}{2 b} .
$$

¿From $1 / 2 \leq 1 / a \leq 1-\delta$ we go to $1 / a^{\prime} \in[\delta, 1 / 2]$ by duality. Therefore, if $4 / 3 \leq$ $b \leq 2$, the convergence

$$
\lim _{k n} S_{k n} F=F, \quad \forall F \in L^{a},
$$

takes place in $L^{a}$ for all $a \in\left[\frac{2 b}{3 b-2}, \frac{2 b}{2-b}\right]$. Proposition 8.9 is proven.

\section{Comments; Conclusion.}

In conclusion of this paper we make a few observations on how far its results could be extended.

1. A potential $\left(\begin{array}{ll}0 & p \\ q & 0\end{array}\right)$ was assumed to be in $L^{2}$, or in $L^{b}, 1<b$. Young inequality (Lemma 5.1) helped to guarantee that the series

$$
\sum \frac{\left|p_{k}\right|+\left|q_{k}\right|}{1+|k|}, \quad \sum \frac{\left|p_{k}\right|+\left|q_{k}\right|}{1+|k|} \cdot \log (2+|k|)
$$

converge; it was a crucial element of the proof of Prop. 2.4 and what followed. But it happens even if we weaken assumptions on $V$ and use the following.

LEMma 9.1. Let $\left(\varphi_{k}\right)$ be a complete orthonormal system on $[0,1]$ which is uniformly bounded, i.e., $\left\|\varphi_{k}\right\|_{\infty} \leq A<\infty$. Then for any function $f \in L(\log L)^{\alpha}, \alpha \geq 1$

$$
\sum \frac{\left|f_{k}\right|}{1+|k|} \cdot(\log (2+|k|))^{\alpha-1} \leq K<\infty,
$$

$K$ depend on $\int_{0}^{1}|f|(\log |f|)_{+}^{\alpha} d x$ only.

See Zygmund [25], v.2, Ch. 12, Misc. \# 8.

If we consider Banach-Orlicz spaces $L(\log L)$ or $L(\log L)^{2}$ we can state an analogue of Lemma 8.2.

Lemma 9.2. Let $Q$ be a compact set in $L(\log L)^{\alpha}, \alpha \geq 1$. Then a sequence $\Omega \in(1.7)$ and $M<\infty$ could be chosen in such a way that

$$
\sum \frac{\Omega_{k}\left|f_{k}\right|}{1+|k|} \cdot(\log (2+|k|))^{\alpha-1} \leq M<\infty
$$

for any $f \in Q$.

Proof is an analogue of the proof of Lemma 8.2, and we omit details.

2. Lemma 9.1 and its incorporation into our construction of Sections 1-4 lead to slight improvement of Thm 4.1 and 4.2 .

Proposition 9.3. (comp. Thm 4.1). Let us consider $L_{b c}, b c=\mathrm{Per}^{ \pm}$or $b c=$ Dir but $V \in \mathcal{H}^{+}$, and assume that

$$
\|V|L(\log L)\|\leq m, \quad\| V| H(\Omega ; L \log L \| \leq M .
$$


There are functions $g=g(m)$ and $N=N(\Omega ; m, M)$ and a sequence $\left\{\delta_{n}\right\}, \delta_{n} \rightarrow$ $0(n \rightarrow \pm \infty)$ depending on $\Omega, m, M$ such that for anuy $V \in(9.4)$ the spectrum of its Dirac operator lies in

$$
R(X, Y) \cup \bigcup_{|n|>N} D\left(n ; \delta_{n}\right),
$$

where $R, D$ are defined in (4.2)-(4.5), with $Y=g(m), X=\pi N+\pi / 2$.

If we consider $L_{b c}, b c=$ Dir, without any assumptionon "evenness" of the potential $V$, we have the same statement with $L(\log L)$ changed to $L(\log L)^{2}$.

3. Other statements on convergence of spectral expansions in Sect. 6-8 put restrictions on $V \in L^{b}$, with $b$ sometimes not close to 1 anyway. For example, the conditions (6.54)-(6.56) of Thm 6.2 imply the following.

Proposition 9.4. ) If $V \in L^{b}$ then the spectral expansions (6.21), or (6.105) converge in $L^{a}$ if

(i) $b \geq 4 / 3,1<a<\infty$,

(ii) $1<b<\frac{4}{3},\left|\frac{1}{a}-\frac{1}{2}\right|<2\left(\frac{1}{b}-\frac{3}{4}\right)$.

Are restrictions (ii) sharp? For example, let $b=8 / 7$; then (ii) becomes $1 / 4<$ $1 / a<3 / 4$. Is it true that for any $\varepsilon>0$ there exists such a potential $V \in L^{8 / 7}$ that its projectors $\left\|S_{n}: L^{4 / 3-\varepsilon} \rightarrow L^{4 / 3-\varepsilon}\right\|$ are unboundeed in $L^{4 / 3-\varepsilon}$ ?

In the same way we can ask questions whether restrictions (a) and (b) in Prop. 7.3 are sharp. We do not write these questions explicitly. But we'll ask whether Thm 8.8 is sharp.

Let us consider

$$
Q=\left\{V \in L^{2}:\left\|V\left|L^{2}\|\leq m, \quad\| V\right| H(\Omega] \mid \leq M\right\}\right.
$$

and assume that for any $V \in Q$ the spectral decompositions (6.105) converge in $L^{2}$ unconditionally for any $F \in L^{2}$. Does it imply that

$$
\sum 1 / \Omega_{k}^{2}<\infty ?
$$

\section{References}

[1] M. A. Ablowitz and H. Segur, Solitons and the inverse scatering transform, SIAM, 1981.

[2] N. K. Bari, Biorthogonal systems aand bases in Hilbert spaces, Uchen. Zapiski M.G.U., 148, 4 (1951), 68 - 107 (Russian).

[3] P. Djakov and B. Mityagin, Smoothness of Schroedinger operator potential in the case of Gevrey type asymptotics of the gaps, J. Funct. Anal. 195 (2002). $89-128$.

[4] P. Djakov and B. Mityagin, Spectral triangles of Schroedinger operators with complex potentials, Selecta Math., New ser. 9 (2003), 495 - 528.

[5] P.Djakov and B. Mityagin, Spectra of 1-D periodic Dirac operators and smoothness of potentials, C.R. Math. Rep. Acad. Sci. Canada vol. 25, (4), (2003), 121 - 125.

[6] I.M.Gelfand, Eigenfunction expansions for an equation with periodic coefficients, Dokl. Akad. Nauk SSSR, 73 (1950), 1117 - 1120 (Russian).

[7] I.C.Gohberg and M.G.Krein, introduction to the theory of linear nonselfadjoint operators, "Nauka", Moscow, 1965; English transl., Transl. Math. Monographs, vol. 18, Amer. Math. Soc., Providence, R.I., 1969; MR 36 \# 3137.

[8] B. Grebert, T. Kappeler and B. Mityagin, Gap estimates of the spectrum of the ZakharovShabat system, Appl. Math. Lett. 11 (1998), 95 - 97.

[9] B. Grebert and T. Kappeler, Estimates on periodic and Dirichlet eigenvalues for the Zakharov - Shabat system, Asympt. Anal. 25 (2001), 201 - 237. 
[10] G.M.Keselman, On the absolute convergence of expansions in eigenfunctions of certain differential operators, Izv. Vuzov SSSR, Mat. 2 (1964), 82 - 93 (Russian).

[11] M. G. Krein and M. A. Krasnoselskii, Fundamental theorems on the extension of Hermitian operators, Uspekhi Mat. Nauk, 2:3 (19) (1947), 60 - 117.

[12] M. G. Krein, M.A.Krasnoselskii and D. P. Milman, On the deficiency indices of operators in Banach space and on some geometric questions, Sborn. Trudov Inst. Mat. Akad. Nauk, Ukr. SSR, 11 (1948), 97 - 112 (Russian).

[13] B. M. Levitan and I. S. Sargsian, Introduction to Spectral theory; Selfadjoint ordinary differential operators, Transl. Math. Monogr. 39, Amer. Math. Soc., R.I., Providence, 1975.

[14] Y. Li and D. McLaughlin, Morse and Melnikov functions for NLS PDEs, Comm. Math. Phys. $162(1994), 175-214$

[15] V. A. Marchenko, Sturm - Liouville operators and applications, Oper. Theory Adv. Appl., vol. 22, Birkhauser, 1986.

[16] A.S.Markus, On an expansion in root vectors of a weakly perturbed self-adjoint operator, Dokl. Akad. Nauk SSSR, 3, 142 (1962), 538 - 541.

[17] A. S. Markus, Some criteria for completeness of root vectors of a linear operator and on summability of series in this system, Dokl. Akad. Nauk SSSR, 4, 155 (1964), 753 - 756 (Russian).

[18] V. P. Mikhailov, On Riesz bases in $L^{2}(0,1)$, Doklady Akad. Nauk SSSR 25, 144 (1962) (Russian).

[19] B. Mityagin, Convergence of spectral decompositiona of periodic Dirac operator, Doklady Akad. Nauk 393, No. 4 (2003)

[20] M. A. Naimark, Linear differential operators, GITTL, Moscow, 1954; English transl., Ungar, New York, 1968.

[21] J. Pöshcel, E. Trubowitz, Inverse spectral theory, Academic Press, 1987.

[22] Exact theory of two-dimensional self-focusing and one-dimensional self-modulation of waves in nonlinear media, Sov. Phys. JETP, 34, 62-69.

[23] A scheme of integrating the non-linear equations of mathematical physics by the method of the invrse scattering problem, I, Funct. Anal. Appl., 8, 226-235.

[24] A scheme of integrating the non-linear equations of mathematical physics by the method of the invrse scattering problem, II, Funct. Anal. Appl., 13, 166-174.

[25] A. Zygmund, Trigonometric Series, London, Cambridge Univ. Press, 1968.

Department of Mathematics, The Ohio State University, Columbus, OH 43210

E-mail address: borismit@math.ohio-state.edu 